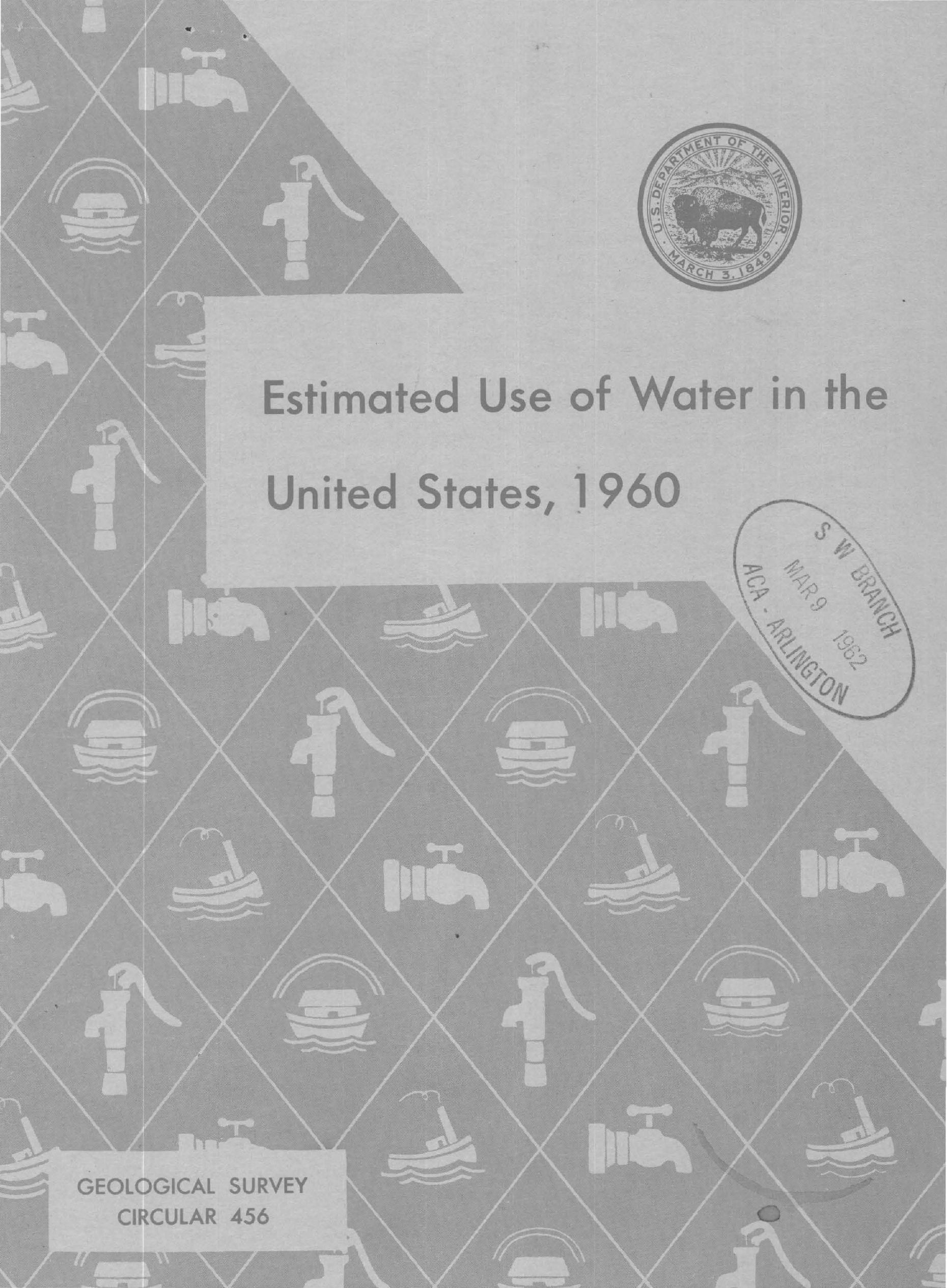





\title{
Estimated Use of Water in the United States, 1960
}

\author{
By K. A. MacKichan and J. C. Kammerer
}

9

Geological SuRvey Circular 456 
$\because \quad \because$ 


\begin{tabular}{|c|c|c|}
\hline \multicolumn{3}{|c|}{ Page } \\
\hline Abstract _...... & 1 & Withdrawal use-Continued \\
\hline Introduction & 1 & Self-supplied industrial use-Continued \\
\hline Previous investigations _. & 1 & Fuel-electric power (public utility)_ 17 \\
\hline Present investigation & 3 & Air conditioning \\
\hline Definition of terms. & 3 & Waterpower. \\
\hline Withdrawal use & 4 & Summary of withdrawal uses \\
\hline Public supplies & 4 & Nonwithdrawal uses \\
\hline Rural _............... & 4 & Changes since 1955 \\
\hline Irrigation $\ldots$ & 13 & Supply versus demand \\
\hline Self-supplied industrial use & 17 & Selected references \\
\hline
\end{tabular}

Figure 1. Map of the United States showing names and locations of water-

Page use regions..........

2. Water used for irrigation (including conveyance losses), by regions in the United States 1960

3. Use of self-supplied industrial water, by regions, in the United States $1960 \ldots$

4. Most industrial water is used for cooling _

5. How water was used in 1960

6. Where water is consumed

7. Trends in use of water for waterpower and all other uses

8. Trends in water used for public supplies, rural supplies, irrigation, and industry

9. Supply and demand in the Nation

10. Supply and demand in the West

\section{TABLES}

Table 1. Water used for public supplies, by States, 1960

2. Water used for public supplies, by regions, 1960

3. Water for rural use, by States, 1960

4. Water for rural use, by regions, $1960 \ldots$

5. Water used for irrigation, by States, $1960 \ldots \ldots \ldots$

6. Water used for irrigation, by regions, $1960 \ldots$

7. Self -supplied industrial water, by States, $1960 \ldots \ldots \ldots$

8. Self -supplied industrial water, by regions, $1960 \ldots \ldots 2$

9. Water used for public utility generation of fuel-electric power, by States, $1960 \ldots \ldots 26$

10. Water used for public utility generation of fuel-electric power, by regions, $1960 \ldots 28$

11. Water withdrawn for air conditioning, by States, 1960

12. Water withdrawn for air conditioning, by regions, 1960

13. Water used for waterpower, by States, 1960

14. Water used for waterpower, by regions, $1960 \ldots$

15. Summary of water withdrawn except for waterpower, by States, $1960 \ldots$

16. Summary of water withdrawn except for waterpower, by regions, $1960 \ldots$

17. Changes in withdrawals 1950-60

18. Supply compared with demand 



\title{
Estimated Use of Water in the United States, 1960
}

\author{
By K. A. MacKichan and J. C. Kammerer
}

\section{ABSTRACT}

The estimated average withdrawal use of water in the United State's during 1960 was almost $270,000 \mathrm{mgd}$ (million gallons per day), exclusive of water used to develop waterpower. This estimated use amounts to about 1,500 gpd (gallons per day) per capita. An additional 2,000,000 mgd were used to develop waterpower.

Withdrawal use of water requires that the water be removed from the ground or diverted from a stream or lake. In this report the use is divided into five types: public supplies, rural, irrigation, self-supplied industrial, and waterpower. Consumptive use of water is the quantity discharged to the atmosphere or incorporated in the products of the process in which it was used. Only $61,000 \mathrm{mgd}$ of the $270,000 \mathrm{mgd}$ withdrawn was consumed.

Of the water withdrawn in 1960, 220,000 mgd (including irrigation conveyance losses) was taken from surface sources and 47,000 from underground sources. Withdrawal of water for uses other than waterpower has increased 12 percent since 1955. The amount of water used for generation of waterpower has increased 33 percent since 1955. The use of saline water was almost twice as great in 1960 as in 1955.

The upper limit of our water supply is the average annual runoff, nearly 1,200,000 mgd. The supply in 1960 was depleted by 61,000 mgd, the amount of water consumed. However, a large part of the water withdrawn but not consumed was deteriorated in quality.

\section{INTRODUCTION}

The use of water generally reduces the water resources and frequently deteriorates the quality of the water, whether the use is withdrawal or nonwithdrawal, consumptive or nonconsumptive. The water quality may be deteriorated by the addition of the following: wastes containing high concentrations of dissolved solids, wastes containing organic matter, bacterial active wastes, and heat. Because the use of water affects the resource, an adequate evaluation of the water resources of the Nation requires a knowledge of the quantity of water used, where it is used, and the type of use. The purpose of this report is to evaluate the use of water during 1960 in broad categories and in States and regions. (See fig. 1.)

\section{PREVIOUS INVESTIGATIONS}

Many Federal, State, and local agencies are interested in data on the use of water, and in recent years a number of agencies have compiled water-use data. The U.S. Public Health Service (1959), under the auspices of the Inter-Agency Committee on Water Resources, Subcommittee on Hydrology, has compiled a report on Federal programs for collection of data on water use. Some investigators made inventories or estimates of water used in a single category. The U.S. Public Health Service (1948), in cooperation with the sanitary-engineering divisions of the State health departments, made inventories of public water-supply facilities in 1945 which included data on the quantity of water used. Similar inventories of facilities serving populations of 25,000 or more were made in 1954, 1955, 1956, 1958, and 1960. The U.S. Public Health Service (1960-61) made a complete inventory again in 1958. The American Water Works Association $(1948,1953,1957)$ has made several national surveys of municipal water works, including information on water use for about 500 water systems serving populations of 10,000 or more.

The U.S. Bureau of the Census (1955) made a survey of water used by the manufacturing industries in 1953, as part of their annual survey of manufactures. Additional data on use of water were collected with the 1954 Census of Manufactures. Picton (1952, 1956, and 1960) made estimates of water used in the United States from 1900 to 1950 and 1955 and forecast estimates of future use. Guyton (1950) made an estimate of ground water used in the United States during 1945, and Langbein (1950) made an estimate of the quantity of water used in the United States in 1946 for the generation of hydroelectric power. An inventory made by Mangan and Graham (1953) 


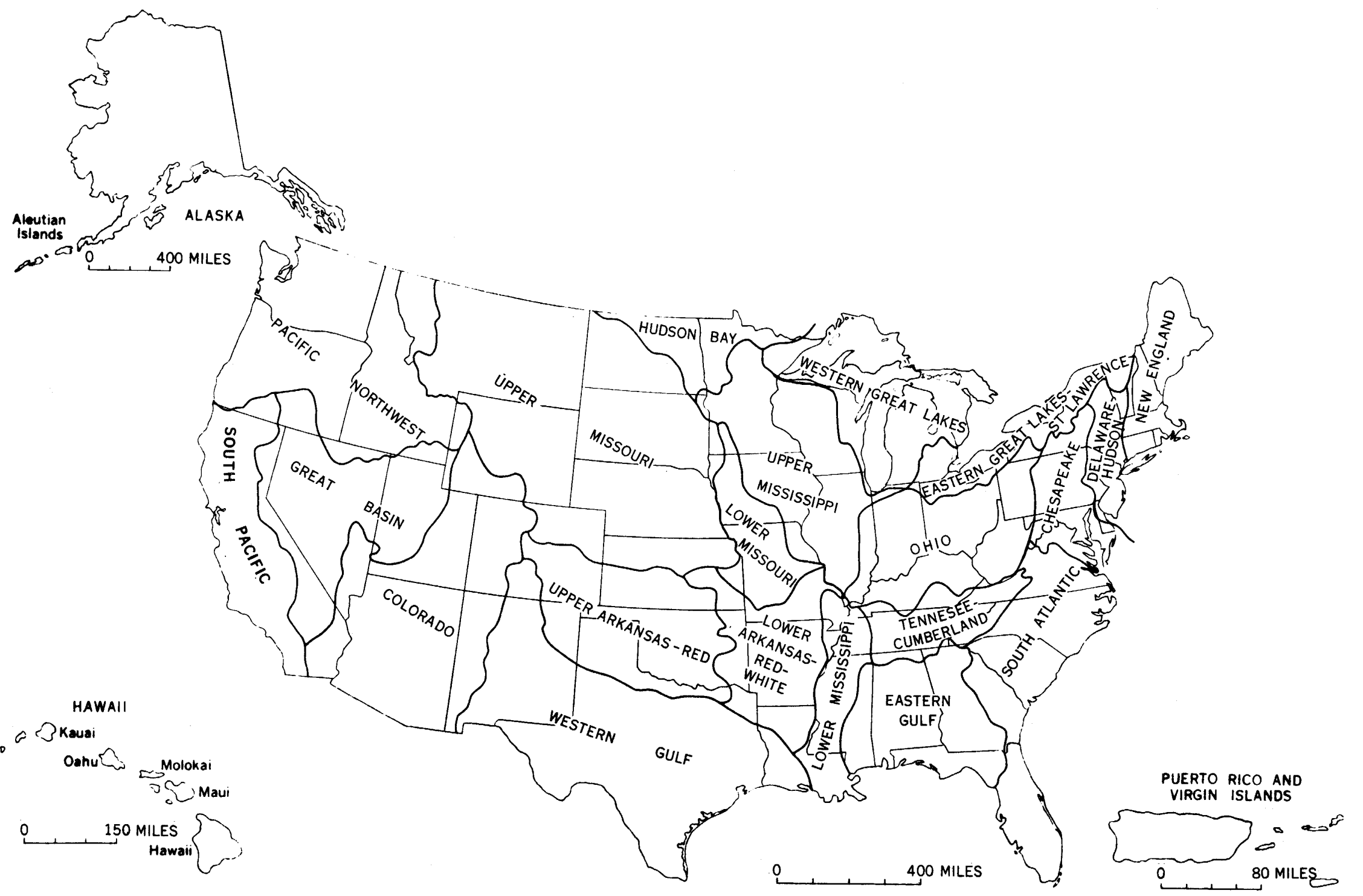


showed the quantity of water used in Pennsylvania in 1951, and Kammerer (written communication, 1961) estimated water use in 1955 for the Delaware River basin. The U.S. Federal Power Commission (1957b) inventoried the water requirements of utility steam electric generating plants in 1954. MacKichan (1951 and 1957) made estimates of the quantity of water used in each of the 48 States during 1950 and 1955 for five major uses: rural, public supplies, industry, irrigation, and waterpower. He also gave the quantity used in 1955 in 19 major regions. MacKichan and Kammerer (1961) estimated water use in 1959-60 for Georgia and parts of adjacent States as a contribution to the water resources investigations being conducted by the U.S. Southeast Area Study Commission.

Woodward (1957) projected water needs for rural supplies, public supplies, self-supplied industry, and irrigation to 1980. The Select Committee on National Water Resources, United States Senate (1959a, 1959b, 1960a, $1960 \mathrm{~b}, 1960 \mathrm{c}, 1960 \mathrm{~d}$, and $1960 \mathrm{e}$ ) published information on current use of water and gave projections to 1980 and 2000.

Among the many reports which give wateruse data for all the principal withdrawal uses within a single State are those for Arkansas (Wood, 1959), California (California Water Resources Board, 1955), Connecticut (Connecticut Water Resources Commission, 1957), Delaware (Smith and others, 1960), Georgia (Thomson and others, 1956), Indiana (Indiana Water Resources Study Committee, 1956), Kansas (Foley, Smrha, and Metzler, 1955), Kentucky (Kentucky Water Resources Commission, 1959), New York (Temporary New York State Commission on Water Resources Planning, 1960), Ohio (Rudnick, 1959), Tennessee(Tennessee Water Resources Division, 1961 ), Virginia (Virginia Advisory Legislative Council, 1955), and Wisconsin (Wirth, 1959).

\section{PRESENT INVESTigation}

This report presents an estimate of the quantity of water withdrawn and the quantity consumed in 1960. It is similar in objective and scope to MacKichan's earlier estimates (1951 and 1957). Some water was withdrawn from a source, used, and discharged into a stream or the ground, only to be withdrawn again. Each time the water was withdrawn it was added to the accumulated total; therefore, the same water was withdrawn several times and was counted each time that it was withdrawn. However, if the water was withdrawn and recirculated so that it was used several times in the same plant before it was discharged into a stream or the ground, it was counted only once. Although the best information available was used, the estimates in this report are, in general, only approximations of the quantity of water used. The estimate for municipal use is probably the most accurate and that for industrial use the least accurate.

District offices of the Water Resources Division of the Geological Survey supplied estimates of water used for public supplies and for manufacturing and nonmanufacturing industries. The estimates were based on data in the Geological Survey files and those furnished by State and local officials. The quantities of water used for power generation at fuel-electric utilities, for rural domestic and stock use, and for irrigation, were computed using statistics of the U.S. Bureau of the Census (1952, 1956, and 1960c), the U.S. Federal Power Commission (1957b, and 196061 ), and the U.S. Department of Agriculture (1960). Information on the sources of water (ground water, surface water, or sewage) was furnished by the district offices of the Water Resources Division of the Geological Survey.

\section{DEFINITION OF TERMS}

Uses of water may be classified in several different ways: among them are withdrawal and nonwithdrawal, consumptive and nonconsumptive uses. Withdrawal uses require that the water be removed from the ground or diverted from a stream or lake. Irrigation, domestic, stock, public, and industrial uses are of this type. Generation of waterpower is also considered a withdrawal use; even in run-of-river plants the water is diverted through the turbines and frequently the generation of waterpower has a very definite effect on the rate of streamflow. The quantity of water withdrawn at a place is the entire quantity of water taken for use. This quantity is sometimes termed "pumpage," "water intake," "duty of water," or "water requirement" (Am. Water Works Assoc. Task Group, 1953). Nonwithdrawal uses do not require diversion. Navigation, recreation, waste disposal, and conservation of fish and wildlife are examples of nonwithdrawal uses. 
The water user either purchases the water from a public-supply system or withdraws it directly from the source for his own use. The latter is self supplied.

Consumptive use is the quantity of water discharged to the atmosphere (evaporated) or incorporated in the products of the process in connection with vegetative growth, food processing, or incidental to an industrial process (Am. Water Works Assoc. Task Group, 1953).

Saline water has been defined as water containing more than $1,000 \mathrm{ppm}$ (parts per million) of dissolved solids regardless of composition. Sodium chloride may not be the principal salt in the water. (Krieger, Hatchett, and Poole, 1956.)

Quantities of water given in this report are generally in million gallons per day (mgd); however, some quantities are also given in acre-feet per year. An acre-foot of water will cover an acre to a depth of 1 foot; 1,000 acre-feet per year equals $0.89 \mathrm{mgd}$.

\section{WITHDRAWAL USE}

Withdrawal uses can be evaluated quantitatively because they require removal of the water from the ground, stream, lake, or reservoir. The total quantity of water withdrawn can be obtained by adding together the known amounts of withdrawals. The primary withdrawal uses are public supplies, rural domestic and stock, irrigation, self-supplied industrial, and waterpower. Fuel-electric utilities and air conditioning are important subdivisions of primary withdrawal uses and have been evaluated. Withdrawal use may be further subdivided into consumptive and nonconsumptive use. The water consumed has been estimated.

\section{PUBLIC SUPPLIES}

Public water-supply systems in the United States served about 136 million people an average of $151 \mathrm{gpd}$ per person or nearly 21,000 mgd. (See tables 1 and 2.)

Water used for public supplies includes all water pumped into the system. This water may be used for fire protection, street flushing, irrigation of lawns and gardens, and by industry and commerce, as well as for domestic supply. Because the water is measured at the source, leakage is also included. Industry and commerce used slightly more than $6,600 \mathrm{mgd}$ from public supplies of which about $560 \mathrm{mgd}$ was used for air conditioning. Of the $20,600 \mathrm{mgd}$ withdrawn for public supplies, almost 3,500 mgd or about 17 percent was consumed. Public water-supply systems may be either publicly or privately owned.

\section{RURAL}

Rural use of water totaled about $3,600 \mathrm{mgd}$, of which $1,600 \mathrm{mgd}$ was used by livestock and $2,000 \mathrm{mgd}$ was for domestic use. (See tables 3 and 4.) Rural homes are defined as those not served by public water-supply systems. About 2,800 mgd was obtained from wells and springs and only $850 \mathrm{mgd}$ was obtained from lakes, streams, and ponds. The resource was depleted by almost $2,800 \mathrm{mgd}$ by this use because practically all the water withdrawn was evaporated or transpired after being discharged on or immediately below the surface of the ground.

The $1,600 \mathrm{mgd}$ of livestock water does not include large quantities of water evaporated from stock ponds. In some areas water thus evaporated may equal or exceed the water consumed by the stock. For example, in North Dakota there are 35,000 stock-water ponds having an average surface area of about 1.5 acres (Erskine, written communication). Assuming an evaporation loss of 2.8 feet per year, the average daily evaporation amounts to about $130 \mathrm{mgd}$. Water consumed by livestock in North Dakota amounted to only 28 mgd.

The rural use of water was computed by multiplying per capita uses by the human and livestock population. Of the 182 million people living in the United States, 48 million supply their own water. Of this 48 million, 36 million have running water in their homes.

Frank (1955) states that people living in the average electrified farm or urban home in the United States use an average of $60 \mathrm{gpd}$ or more per person for household purposes and watering of lawns. The corresponding average for homes without running water is only $10 \mathrm{gpd}$ per person. Other investigators report that only 50 gpd per person is used in homes with running water. Quantities of rural 
Table 1. -Water used for public supplies, by States, 1960

\begin{tabular}{|c|c|c|c|c|c|c|c|c|c|c|c|c|}
\hline & \multicolumn{3}{|c|}{ Population served } & \multicolumn{4}{|c|}{ Water withdrawn } & \multicolumn{4}{|c|}{ Water delivered } & \multirow{3}{*}{$\begin{array}{l}\text { Water } \\
\text { con- } \\
\text { sumed } \\
\text { (mgd) }\end{array}$} \\
\hline & \multirow{2}{*}{$\begin{array}{c}\text { Ground } \\
\text { water } \\
\text { (thousands) }\end{array}$} & \multirow{2}{*}{$\begin{array}{c}\text { Surface } \\
\text { water } \\
\text { (thousands) }\end{array}$} & \multirow{2}{*}{$\begin{array}{c}\text { All } \\
\text { water } \\
\text { (thousands) }\end{array}$} & \multirow{2}{*}{$\begin{array}{c}\text { Ground } \\
\text { water } \\
\text { (mgd) }\end{array}$} & \multirow{2}{*}{$\begin{array}{c}\text { Surface } \\
\text { water } \\
\text { (mgd) }\end{array}$} & \multirow{2}{*}{$\begin{array}{c}\text { All } \\
\text { water } \\
\text { (mgd) }\end{array}$} & \multirow{2}{*}{$\begin{array}{c}\text { Per } \\
\text { capita } \\
\text { (gpd) }\end{array}$} & \multicolumn{3}{|c|}{ Industrial and commercial uses } & \multirow{2}{*}{$\begin{array}{l}\text { Domestic } \\
\text { use and } \\
\text { losses } 1 \\
\text { (mgd) }\end{array}$} & \\
\hline & & & & & & & & $\begin{array}{c}\text { Air } \\
\text { conditioning } \\
\text { (mgd) }\end{array}$ & $\mid \begin{array}{c}\text { Except air } \\
\text { conditioning } \\
\text { (mgd) }\end{array}$ & $\begin{array}{c}\text { All } \\
\text { uses } \\
\text { (mgd) }\end{array}$ & & \\
\hline Alabama ...... & 808 & 1,200 & 2,000 & 84 & 150 & 230 & 118 & 9.4 & 69 & 78 & 160 & 54 \\
\hline Alaska ......- & 31 & 43 & 74 & 7.9 & 15 & 23 & 307 & 0 & 10 & 10 & 13 & .3 \\
\hline Arizona _...... & 697 & 283 & 980 & 100 & 42 & 150 & 150 & 6.4 & 15 & 21 & 130 & 74 \\
\hline Arkansas . . . - & 457 & 478 & 935 & 48 & 51 & 100 & 107 & 0 & 27 & 27 & 73 & 18 \\
\hline California .... & 5,800 & 7,310 & 13,100 & 1,200 & 1,400 & 2,600 & 201 & 76 & 300 & 380 & 2,300 & 370 \\
\hline Colorado _....- & 209 & 1,290 & 1,500 & 41 & 250 & 290 & 196 & 9.1 & 69 & 78 & 220 & 69 \\
\hline Connecticut ... & 115 & 1,970 & 2,090 & 14 & 240 & 260 & 122 & 6.9 & 90 & 97 & 160 & 77 \\
\hline Delaware ..... & 134 & 176 & 310 & 11 & 28 & 40 & 128 & 1.6 & 20 & 22 & 18 & 4.0 \\
\hline Florida & 3,320 & 54 & 3,370 & 500 & 23 & 530 & 156 & 7.5 & 142 & 150 & 370 & 140 \\
\hline Georgia . & 7.11 & 1,600 & 2,310 & 110 & 260 & 370 & 161 & 8.1 & 71 & 79 & 290 & 33 \\
\hline Hawaii . . . & 511 & 70 & 581 & 74 & 11 & 85 & 146 & 5.0 & 25 & 30 & 55 & 25 \\
\hline Idaho & 352 & 97 & 449 & 91 & 28 & 120 & 264 & 1.5 & 54 & 56 & 62 & 18 \\
\hline Illinois & 2,860 & 5,600 & 8,470 & 200 & 1,400 & 1,600 & 186 & 67 & 320 & 390 & 1,200 & 160 \\
\hline Indiana & 1,230 & 1,680 & $2,9.10$ & 130 & 230 & 370 & 126 & 29 & 110 & 140 & 220 & 92 \\
\hline lowa & 943 & 555 & 1,500 & 91 & 68 & 160 & 106 & 5.1 & 67 & 72 & 88 & 16 \\
\hline Kansas ..... & 734 & 583 & 1,320 & 120 & 81 & 200 & 150 & .8 & 24 & 25 & 170 & 69 \\
\hline Kentucky -.- & 251 & 1,400 & 1,660 & 18 & 210 & 230 & 138 & 1.3 & 71 & 72 & 160 & 23 \\
\hline Louisiana _._. & 1,020 & 1,140 & 2,150 & 91 & 180 & 270 & 126 & 27 & 54 & 81 & 190 & 110 \\
\hline Maine & 136 & 608 & 744 & 11 & 75 & 86 & 116 & 1.0 & 30 & 31 & 55 & 17 \\
\hline Maryland ....- & 190 & 2,200 & 2,390 & 21 & 280 & 300 & 127 & 12 & 100 & 110 & 200 & 22 \\
\hline Massachusetts_ & 1,300 & 3,640 & 4,930 & 120 & 470 & 590 & 119 & 8.3 & 170 & 170 & 420 & 59 \\
\hline Michigan & 1,310 & 4,230 & 5,540 & 170 & 670 & 840 & 153 & 28 & 410 & 440 & 400 & 94 \\
\hline Minnesota _.... & 1,080 & $1,07,0$ & 2,140 & 100 & 120 & 220 & 104 & 10 & 60 & 70 & 150 & 29 \\
\hline Mississippi _... & 777 & 238 & 1,020 & 87 & 28 & 110 & 113 & 4.0 & 28 & 32 & 83 & 39 \\
\hline Missouri & 460 & 2,300 & 2,760 & 49 & 370 & 410 & 150 & 12 & 180 & 190 & 230 & 80 \\
\hline Montana & 96 & 356 & 452 & 24 & 89 & 110 & 250 & 1.0 & 18 & 19 & 94 & 56 \\
\hline Nebraska & 648 & 342 & 990 & 120 & 58 & 180 & 178 & 3.9 & 72 & 76 & 100 & 8.3 \\
\hline Nevada & 139 & 89 & 228 & 43 & 36 & 79 & 346 & .71 & 13 & 14 & 65 & 42 \\
\hline
\end{tabular}

See footnote at end of table. 
Table 1.-Water used for public supplies, by States, 1960-Continued

\begin{tabular}{|c|c|c|c|c|c|c|c|c|c|c|c|c|}
\hline & \multicolumn{3}{|c|}{ Population served } & \multicolumn{4}{|c|}{ Water withdrawn } & \multicolumn{4}{|c|}{ Water delịvered } & \multirow{3}{*}{$\begin{array}{l}\text { Water } \\
\text { con- } \\
\text { sumed } \\
\text { (mgd) }\end{array}$} \\
\hline & \multirow{2}{*}{$\begin{array}{c}\text { Ground } \\
\text { water } \\
\text { (thousands) }\end{array}$} & \multirow{2}{*}{$\begin{array}{c}\text { Surface } \\
\text { water } \\
\text { (thousands) }\end{array}$} & \multirow{2}{*}{$\begin{array}{c}\text { All } \\
\text { water } \\
\text { (thousands) }\end{array}$} & \multirow{2}{*}{$\begin{array}{c}\text { Ground } \\
\text { water } \\
\text { (mgd) }\end{array}$} & \multirow{2}{*}{$\begin{array}{c}\text { Surface } \\
\text { water } \\
\text { (mgd) }\end{array}$} & \multirow{2}{*}{$\begin{array}{c}\text { All } \\
\text { water } \\
\text { (mgd) }\end{array}$} & \multirow{2}{*}{$\begin{array}{c}\text { Per } \\
\text { capita } \\
\text { (gpd) }\end{array}$} & \multicolumn{3}{|c|}{ Industrial and commercial uses } & \multirow{2}{*}{$\begin{array}{l}\text { Domestic } \\
\text { use and } \\
\text { losses } \\
\text { (mgd) }\end{array}$} & \\
\hline & & & & & & & & $\begin{array}{c}\text { Air } \\
\text { conditioning } \\
\text { (mgd) }\end{array}$ & $\begin{array}{c}\text { Except air } \\
\text { conditioning } \\
\text { (mgd) }\end{array}$ & $\begin{array}{c}\text { All } \\
\text { uses } \\
\text { (mgd) }\end{array}$ & & \\
\hline New Hampshire & 222 & 292 & 514 & 18 & 36 & 54 & 106 & 0.8 & 16 & 17 & 37 & 2.8 \\
\hline New Jersey & 1,610 & 3,080 & 4,700 & 230 & 440 & 670 & 143 & 50 & 150 & 200 & 470 & 20 \\
\hline New Mexico _.. & 556 & 105 & 661 & 84 & 24 & 110 & 163 & .9 & 11 & 12 & 96 & 49 \\
\hline New York & 3,360 & 11,900 & 15,300 & 330 & 1,700 & 2,100 & 136 & 18 & 570 & 590 & 1,500 & 350 \\
\hline North Carolina - & 355 & 1,860 & 2,220 & 34 & 260 & 290 & 131 & 2.9 & 55 & 58 & 230 & 29 \\
\hline North Dakota... & 182 & 172 & 354 & 14 & 19 & 32 & 91 & .3 & 1.9 & 2.2 & 30 & 12 \\
\hline Ohio & 1,900 & 5,440 & 7,330 & 230 & 790 & 1,000 & 138 & 0 & 600 & 610 & 410 & 120 \\
\hline Oklahoma... & 568 & 1,250 & 1,820 & 56 & 150 & 210 & 115 & 15 & 51 & 66 & 140 & 72 \\
\hline Oregon & 284 & 1,100 & 1,380 & 170 & 220 & 390 & 284 & .4 & 130 & 130 & 260 & 36 \\
\hline Pennsylvania_. - & 736 & 8,460 & 9,200 & 110 & 1,200 & 1,300 & 144 & 32 & 640 & 670 & 650 & 120 \\
\hline Puerto Rico & 350 & 1,400 & 1,750 & 6.8 & 61 & 68 & 39 & 1.1 & 21 & 22 & 46 & 11 \\
\hline Rhode Island & 140 & 689 & 829 & 10 & 71 & 81 & 97 & 2.0 & 38 & 40 & 41 & 5.0 \\
\hline South Carolina - & 411 & 967 & 1,380 & 35 & 150 & 190 & 137 & 2.9 & 55 & 58 & 130 & 19 \\
\hline South Dakota _.- & 341 & 71 & 412 & 46 & 8.6 & 54 & 132 & .1 & 4.9 & 5.0 & 50 & 19 \\
\hline Tennessee & 1,220 & 1,190 & 2,410 & 150 & 190 & 340 & 142 & 4.2 & 96 & 100 & 240 & 81 \\
\hline Texas & 4,420 & 4,160 & 8,580 & 530 & 600 & 1,100 & 132 & 37 & 320 & 360 & 780 & 480 \\
\hline Utah $\ldots \ldots$ & 363 & 369 & 732 & 100 & 120 & 220 & 301 & 5.5 & 6 & 12 & 210 & 45 \\
\hline Vermont - & 70 & 160 & 230 & 8.1 & 24 & 32 & 140 & .6 & 12 & 13 & 19 & 1.7 \\
\hline Virginia _... & 304 & 1,900 & 2,200 & 43 & 220 & 260 & 129 & 14 & 110 & 120 & 160 & 29 \\
\hline Virgin Islands & 10 & 14 & 24 & .3 & .6 & & 37 & 0 & .1 & .1 & .8 & .7 \\
\hline Washington & 946 & 1,310 & 2,260 & 200 & 570 & 770 & 341 & 1.7 & 310 & 310 & 460 & 77 \\
\hline West Virginia - - & 450 & 802 & 1,250 & 37 & 87 & 120 & 99 & .3 & 46 & 46 & 78 & 6.6 \\
\hline $\begin{array}{l}\text { Wisconsin } \\
\text { Wyoming }\end{array}$ & $\begin{array}{r}1,390 \\
93\end{array}$ & $\begin{array}{r}1,040 \\
124\end{array}$ & $\begin{array}{r}2,430 \\
217\end{array}$ & $\begin{array}{r}160 \\
33\end{array}$ & $\begin{array}{r}210 \\
26\end{array}$ & $\begin{array}{r}370 \\
59\end{array}$ & $\begin{array}{l}152 \\
271\end{array}$ & $\begin{array}{c}20 \\
2.2\end{array}$ & $\begin{array}{r}150 \\
10\end{array}$ & $\begin{array}{r}170 \\
12\end{array}$ & $\begin{array}{r}200 \\
46\end{array}$ & $\begin{array}{l}37 \\
34\end{array}$ \\
\hline $\begin{array}{l}\text { District of. } \\
\text { Columbia. }\end{array}$ & 0 & 764 & 764 & 0 & 140 & 140 & 185 & 7.1 & 54 & 61 & 81 & 15 \\
\hline
\end{tabular}

See footnote at end of table. 


\begin{tabular}{|c|c|c|c|c|c|c|c|c|c|c|c|c|}
\hline $\begin{array}{l}\text { United States } \\
\text { excluding } \\
\text { Alaska,Hawaii, } \\
\text { Puerto Rico, } \\
\text { and Virgin } \\
\text { Island. }\end{array}$ & 45,700 & 87,700 & 133,000 & 6,240 & 14,100 & 20,400 & 153 & 556 & 6,020 & 6,580 & 13,800 & 3,430 \\
\hline United States _- & 46,600 & 89,200 & 136,000 & 6,330 & 14,200 & 20,600 & 151 & 562 & 6,080 & 6,640 & 13,900 & 3,470 \\
\hline
\end{tabular}

${ }^{1}$ Includes public use.

Table 2.-Water used for public supplies, by regions, 1960

\begin{tabular}{|c|c|c|c|c|c|c|c|c|c|c|c|c|}
\hline & \multicolumn{3}{|c|}{ Population served } & \multicolumn{4}{|c|}{ Water withdrawn } & \multicolumn{4}{|c|}{ Water delivered } & \multirow{3}{*}{$\begin{array}{l}\text { Water } \\
\text { con- } \\
\text { sumed } \\
\text { (mgd) }\end{array}$} \\
\hline & \multirow{2}{*}{$\mid \begin{array}{c}\text { Ground } \\
\text { water } \\
\text { (thousands) }\end{array}$} & \multirow{2}{*}{$\begin{array}{c}\text { Surface } \\
\text { water } \\
\text { (thousands) }\end{array}$} & \multirow{2}{*}{$\mid \begin{array}{c}\text { All } \\
\text { water } \\
\text { (thousands) }\end{array}$} & \multirow{2}{*}{$\begin{array}{l}\text { Ground } \\
\text { water } \\
\text { (mgd) }\end{array}$} & \multirow{2}{*}{$\begin{array}{l}\text { Surface } \\
\text { water } \\
\text { (mgd) }\end{array}$} & \multirow{2}{*}{$\begin{array}{c}\text { All } \\
\text { water } \\
\text { (mgd) }\end{array}$} & \multirow{2}{*}{$\begin{array}{c}\text { Per } \\
\text { capita } \\
\text { (gpd) }\end{array}$} & \multicolumn{3}{|c|}{ Industrial and commercial uses } & \multirow{2}{*}{$\begin{array}{l}\text { Domestic } \\
\text { use and } \\
\text { losses } 1 \\
\text { (mgd) }\end{array}$} & \\
\hline & & & & & & & & $\begin{array}{c}\text { Air } \\
\text { conditioning } \\
\text { (mgd) }\end{array}$ & $\mid \begin{array}{c}\text { Except air } \\
\text { conditioning } \\
\text { (mgd) }\end{array}$ & $\begin{array}{c}\text { All } \\
\text { uses } \\
\text { (mgd) }\end{array}$ & & \\
\hline New England ... & 1,900 & 7,020 & 8,920 & 170 & 870 & 1,000 & 117 & 19 & 320 & 350 & 700 & 150 \\
\hline $\begin{array}{l}\text { Delaware- } \\
\text { Hudson. }\end{array}$ & 4,830 & 16,200 & 21,100 & 550 & 2,400 & 3,000 & 142 & 84 & 920 & 1,000 & 1,970 & 370 \\
\hline Chesapeake _. - & 936 & 5,460 & 6,390 & 120 & 760 & 880 & 135 & 32 & 340 & 370 & 500 & 82 \\
\hline South Atlantic - - & 4,200 & 4,780 & 8,980 & 590 & 690 & 1,300 & 145 & 24 & 390 & 410 & 900 & 200 \\
\hline Eastern Gulf $\ldots-$ & 1,710 & 2,160 & 3,860 & 220 & 280 & 490 & 128 & 17 & 93 & 110 & 390 & 100 \\
\hline $\begin{array}{l}\text { Tennessee - } \\
\text { Cumberland. }\end{array}$ & 600 & 1,570 & 2,170 & 73 & 240 & 320 & 146 & 4.5 & 80 & 84 & 230 & 60 \\
\hline Ohio _....... & 3,640 & 8,720 & 12,400 & 400 & 1,100 & 1,500 & 122 & 27 & 590 & 620 & 880 & 190 \\
\hline $\begin{array}{l}\text { Eastern Great } \\
\text { Lakes-St. } \\
\text { Lawrence. }\end{array}$ & 912 & 9,090 & 10,000 & 93 & 1,400 & 1,500 & 151 & 24 & 800 & 820 & 690 & 200 \\
\hline $\begin{array}{l}\text { Western Great } \\
\text { Lakes. }\end{array}$ & 2,700 & 7,210 & 9,900 & 270 & 1,600 & 1,900 & 192 & 92 & 520 & 610 & 1,300 & 200 \\
\hline Hudson Bay -.-- & 197 & 167 & 364 & 15 & 18 & 33 & 90 & .8 & 4.2 & 5.0 & 28 & 9.0 \\
\hline $\begin{array}{l}\text { Upper } \\
\text { Mississippi. }\end{array}$ & 4,480 & 3,700 & 8,180 & 410 & 600 & 1,000 & 123 & 34 & 350 & 380 & 630 & 130 \\
\hline Upper Missouri & 1,620 & 2,100 & 3,720 & 280 & 380 & 650 & 176 & 14 & 170 & 180 & 470 & 180 \\
\hline Lower Missouri & 389 & 873 & 1,260 & 36 & 130 & 160 & 130 & 3.3 & 60 & 63 & 100 & 32 \\
\hline
\end{tabular}

See footnote at end of table. 
Table 2.-Water used for public supplies, by reaions, 1960-Continued

\begin{tabular}{|c|c|c|c|c|c|c|c|c|c|c|c|c|}
\hline & \multicolumn{3}{|c|}{ Population served } & \multicolumn{4}{|c|}{ Water withdrawn } & \multicolumn{4}{|c|}{ Water delivered } & \multirow{3}{*}{$\begin{array}{l}\text { Water } \\
\text { con- } \\
\text { sumed } \\
\text { (mgd) }\end{array}$} \\
\hline & \multirow{2}{*}{$\begin{array}{c}\text { Ground } \\
\text { water } \\
\text { (thousands) }\end{array}$} & \multirow{2}{*}{$\begin{array}{c}\text { Surface. } \\
\text { water } \\
\text { (thousands) }\end{array}$} & \multirow{2}{*}{$\begin{array}{c}\text { All } \\
\text { water } \\
\text { (thousands) }\end{array}$} & \multirow{2}{*}{$\begin{array}{c}\text { Ground } \\
\text { water } \\
\text { (mgd) }\end{array}$} & \multirow{2}{*}{$\begin{array}{l}\text { Surface } \\
\text { water } \\
\text { (mgd) }\end{array}$} & \multirow{2}{*}{$\left|\begin{array}{c}\text { All } \\
\text { uses } \\
\text { (mgd) }\end{array}\right|$} & \multirow{2}{*}{$\begin{array}{c}\text { Per } \\
\text { capita } \\
\text { (gpd) }\end{array}$} & \multicolumn{3}{|c|}{ Industrial and commercial uses } & \multirow{2}{*}{$\begin{array}{c}\text { Domestic } \\
\text { and use } \\
\text { losses } \\
\text { (mgd) }\end{array}$} & \\
\hline 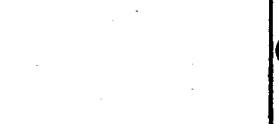 & & & & & & & & $\mid \begin{array}{c}\text { Air } \\
\text { conditioning } \\
\text { (mgd) }\end{array}$ & $\begin{array}{c}\text { Except air } \\
\text { conditioning } \\
\text { (mgd) }\end{array}$ & $\begin{array}{c}\text { All } \\
\text { uses } \\
\text { (mgd) }\end{array}$ & & \\
\hline $\begin{array}{l}\text { Lower } \\
\text { Mississippi. }\end{array}$ & 1,900 & 1,080 & 2,990 & 210 & 170 & 380 & 127 & 23 & 100 & 120 & 260 & 110 \\
\hline $\begin{array}{l}\text { Upper } \\
\text { Arkansas- } \\
\text { Red. }\end{array}$ & 1,310 & 1,210 & 2,520 & 180 & 170 & 350 & 139 & 14 & 59 & 73 & 270 & 110 \\
\hline $\begin{array}{l}\text { Lower } \\
\text { Arkansas- } \\
\text { Red-White. }\end{array}$ & 888 & 1,520 & 2,400 & 85 & 190 & 280 & 117 & 13 & 75 & 88 & 190 & 86 \\
\hline Western Gulf _- & 4,820 & 3,980 & 8,800 & 590 & 590 & 1,200 & 136 & 36 & 320 & 360 & 820 & 520 \\
\hline Colorado.....-. & 898 & 508 & 1,410 & 160 & 110 & 270 & 193 & 8.5 & 25 & 33 & 240 & 120 \\
\hline Great Basin $\ldots$ & 493 & 445 & 938 & 130 & 140 & 280 & 296 & 5.8 & 27 & 33 & 250 & 67 \\
\hline South Pacific -- & 5,710 & 7,250 & 13,000 & 1,300 & 1,400 & 2,700 & 208 & 76 & 400 & 480 & 2,200 & 370 \\
\hline $\begin{array}{l}\text { Pacific North- } \\
\text { west. }\end{array}$ & 1,550 & 2,620 & 4,170 & 350 & 840 & 1,200 & 286 & 3.2 & 390 & 390 & 810 & 150 \\
\hline Hawaii _...... & 511 & 70 & 581 & 74 & 11 & 85 & 146 & 5.0 & 35 & 30 & 55 & 25 \\
\hline Alaska _...... & 31 & 43 & 74 & 7.9 & 15 & 23 & 307 & 0 & 10 & 10 & 13 & .3 \\
\hline $\begin{array}{l}\text { Puerto Rico } \\
\text { and Virgin } \\
\text { Islands. }\end{array}$ & 360 & 1,410 & 1,770 & 7.1 & 62 & 69 & 39 & 1.1 & 21 & 22 & 47 & 11 \\
\hline $\begin{array}{l}\text { United States } \\
\text { excluding } \\
\text { Alaska, } \\
\text { Hawaii, Puertd } \\
\text { Rico, and } \\
\text { Virgin Islands. }\end{array}$ & 45,700 & 87,700 & 133,000 & 6,240 & 14,100 & 20,400 & 153 & 556 & 6,020 & 6,580 & 13,800 & 3,430 \\
\hline United States -- & 46,600 & 89,200 & 136,000 & 6,330 & 14,200 & 20,600 & 151 & 562 & 6,080 & 6,640 & 13,900 & 3,470 \\
\hline
\end{tabular}

\footnotetext{
${ }^{1}$ Includes public use.
} 
Table 3.-Water for rural use, by States, 1960

[Million gallons per day]

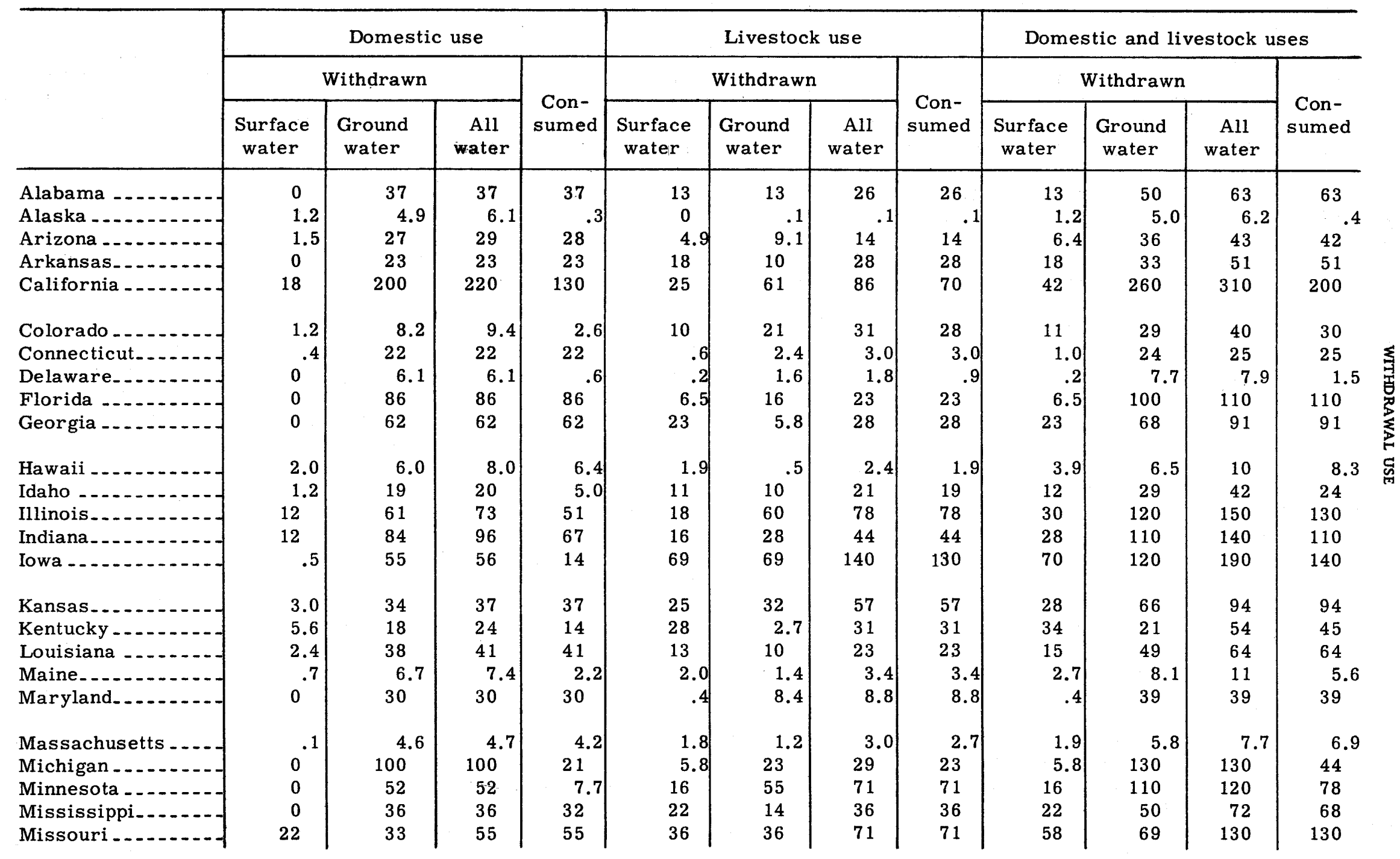


Table 3.-Water for rural use, by States, 1960-Continued

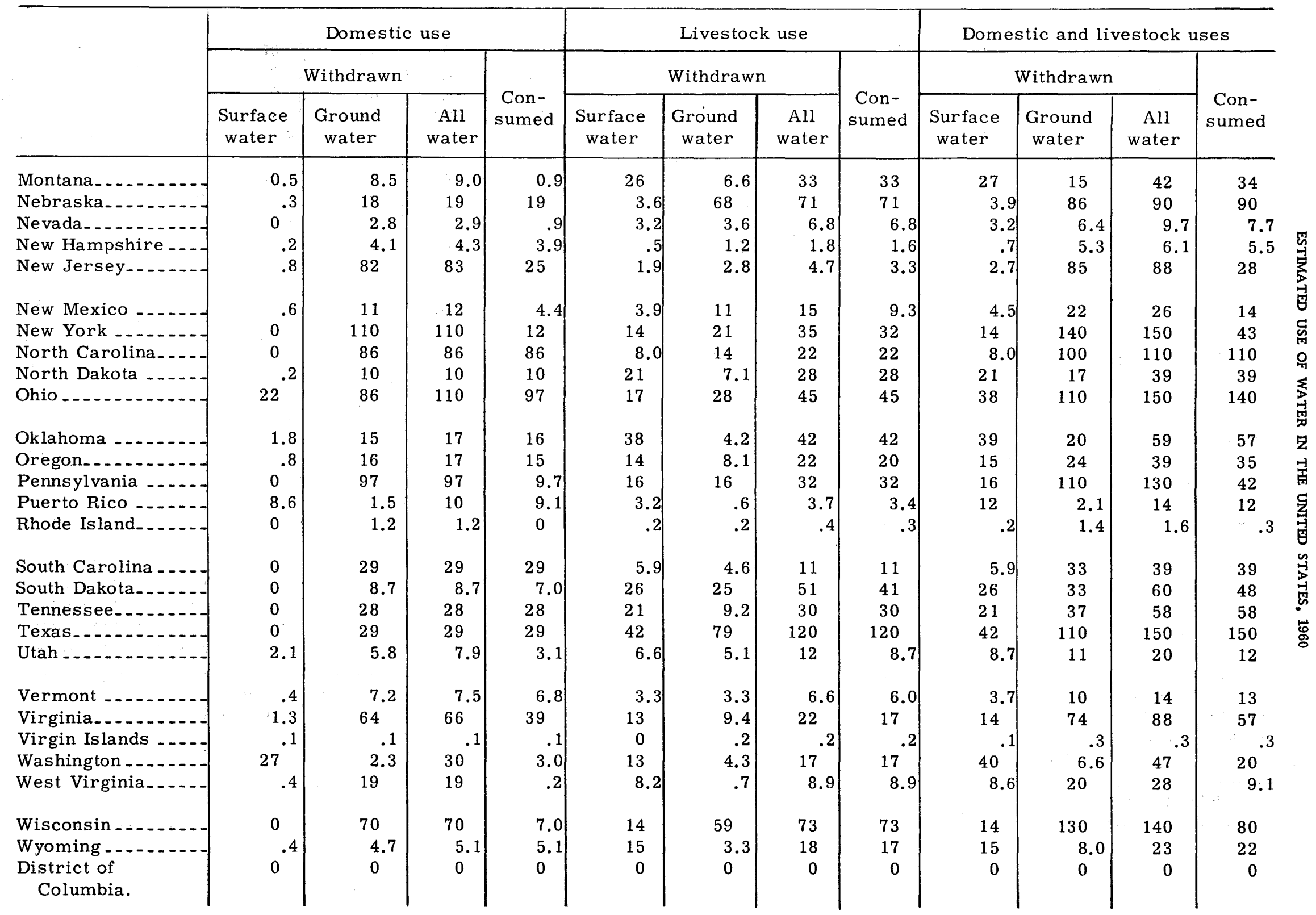




\begin{tabular}{|c|c|c|c|c|c|c|c|c|c|c|c|c|}
\hline $\begin{array}{l}\text { United States ex- } \\
\text { cluding Alaska, } \\
\text { Hawaii, Puerto } \\
\text { Rico, and Virgin } \\
\text { Islands. }\end{array}$ & 140 & 1,900 & 2,000 & 1,200 & 700 & 890 & 1,600 & 1,500 & 840 & 2,800 & 3,600 & 2,700 \\
\hline United States & 150 & 1,900 & 2,000 & 1,200 & 700 & 890 & 1,600 & 1,500 & 850 & 2,800 & 3,600 & 2,800 \\
\hline
\end{tabular}

Table 4.-Water for rural use, by regions, 1960

[Million gallons per day]

\begin{tabular}{|c|c|c|c|c|c|c|c|c|c|c|c|c|}
\hline \multirow{3}{*}{ Region } & \multicolumn{4}{|c|}{ Domestic use } & \multicolumn{4}{|c|}{ Livestock use } & \multicolumn{4}{|c|}{ Domestic and livestock uses } \\
\hline & \multicolumn{3}{|c|}{ Withdrawn } & \multirow{2}{*}{$\begin{array}{c}\text { Con- } \\
\text { sumed }\end{array}$} & \multicolumn{3}{|c|}{ Withdrawn } & \multirow{2}{*}{$\begin{array}{l}\text { Con- } \\
\text { sumed }\end{array}$} & \multicolumn{3}{|c|}{ Withdrawn } & \multirow{2}{*}{$\begin{array}{l}\text { Con- } \\
\text { sumed }\end{array}$} \\
\hline & $\begin{array}{l}\text { Surface } \\
\text { water }\end{array}$ & $\begin{array}{l}\text { Ground } \\
\text { water }\end{array}$ & $\begin{array}{l}\text { All } \\
\text { water }\end{array}$ & & $\begin{array}{l}\text { Surface } \\
\text { water }\end{array}$ & $\begin{array}{l}\text { Ground } \\
\text { water }\end{array}$ & $\begin{array}{c}\text { All } \\
\text { water }\end{array}$ & & $\begin{array}{l}\text { Surface } \\
\text { water }\end{array}$ & $\begin{array}{l}\text { Ground } \\
\text { water }\end{array}$ & $\begin{array}{c}\text { All } \\
\text { water }\end{array}$ & \\
\hline New England....... & 1.6 & 37 & 39 & 31 & 6.1 & 7.3 & 13 & 13 & 7.7 & 45 & 52 & 44 \\
\hline Delaware-Hudson & .9 & 160 & 160 & 37 & 8.1 & 14 & 22 & 19 & 9.0 & 180 & 180 & 56 \\
\hline Chesapeake....... & .5 & 100 & 100 & 49 & 18 & 24 & 42 & 39 & 18 & 120 & 140 & 87 \\
\hline South Atlantic & .4 & 210 & 210 & 200 & 29 & 40 & 69 & 67 & 29 & 250 & 280 & 270 \\
\hline Eastern Gulf & 0 & 110 & 110 & 110 & 38 & 23 & 60 & 60 & 38 & 140 & 170 & 170 \\
\hline $\begin{array}{l}\text { Tennessee- } \\
\text { Cumberland. }\end{array}$ & 1.1 & 57 & 58 & 54 & 28 & 9.9 & 38 & 38 & 29 & 66 & 96 & 91 \\
\hline Ohio & 31 & 190 & 230 & 140 & 69 & 58 & 130 & 130 & 100 & 250 & 350 & 260 \\
\hline $\begin{array}{l}\text { Eastern Great } \\
\text { Lakes-St. } \\
\text { Lawrence. }\end{array}$ & 8.6 & 130 & 130 & 54 & 15 & 23 & 38 & 36 & 24 & 150 & 170 & 90 \\
\hline $\begin{array}{l}\text { Western Great } \\
\text { Lakes. }\end{array}$ & 1.1 & 160 & 160 & 42 & 13 & 41 & 54 & 49 & 14 & 200 & 210 & 90 \\
\hline Hudson Bay....... & .1 & 9.7 & 9.8 & 6.7 & 9.8 & 11 & 21 & 21 & 9.9 & 20 & 30 & 27 \\
\hline Upper Mississippi_ & 17 & 160 & 180 & 73 & 91 & 200 & 290 & 290 & 110 & 360 & 470 & 360 \\
\hline Upper Missouri ... & 2.7 & 65 & 67 & 56 & 96 & 140 & 230 & 220 & 99 & 200 & 300 & 280 \\
\hline Lower Missouri... & 12 & 33 & 45 & 33 & 41 & 42 & 83 & 81 & 53 & 74 & 130 & 110 \\
\hline Lower Mississippi & 4.6 & 50 & 55 & 52 & 23 & 18 & 41 & 41 & 28 & 68 & 95 & 93 \\
\hline $\begin{array}{l}\text { Upper Arkansas - } \\
\text { Red. }\end{array}$ & 2.5 & 29 & 31 & 28 & 43 & 32 & 74 & 72 & 45 & 61 & 110 & 100 \\
\hline
\end{tabular}


Table 4. - Water for rural use, by reqions, 1960-Continued

\begin{tabular}{|c|c|c|c|c|c|c|c|c|c|c|c|c|}
\hline \multirow{3}{*}{ Region } & \multicolumn{4}{|c|}{ Domestic use } & \multicolumn{4}{|c|}{ Livestock use } & \multicolumn{4}{|c|}{ Domestic and livestock uses } \\
\hline & \multicolumn{3}{|c|}{ Withdrawn } & \multirow{2}{*}{$\begin{array}{c}\text { Con- } \\
\text { sumed }\end{array}$} & \multicolumn{3}{|c|}{ Withdrawn } & \multirow{2}{*}{$\begin{array}{l}\text { Con- } \\
\text { sumed }\end{array}$} & \multicolumn{3}{|c|}{ Withdrawn } & \multirow{2}{*}{$\begin{array}{l}\text { Con- } \\
\text { sumed }\end{array}$} \\
\hline & $\begin{array}{l}\text { Surface } \\
\text { water }\end{array}$ & $\begin{array}{l}\text { Ground } \\
\text { water }\end{array}$ & $\begin{array}{c}\text { All } \\
\text { water }\end{array}$ & & $\begin{array}{l}\text { Surface } \\
\text { water }\end{array}$ & $\begin{array}{l}\text { Ground } \\
\text { water }\end{array}$ & $\begin{array}{c}\text { All } \\
\text { water }\end{array}$ & & $\begin{array}{l}\text { Surface } \\
\text { water }\end{array}$ & $\begin{array}{l}\text { Ground } \\
\text { water }\end{array}$ & $\begin{array}{c}\text { All } \\
\text { water }\end{array}$ & \\
\hline $\begin{array}{l}\text { Lower Arkansas- } \\
\text { Red-White. }\end{array}$ & 3.2 & 40 & 43 & 42 & 43 & 24 & 67 & 67 & 46 & 64 & 110 & 110 \\
\hline Western Gulf ..... & .2 & 40 & 40 & 34 & 40 & 78 & 120 & 120 & 40 & 120 & 160 & 150 \\
\hline Colorado & 3.7 & 39 & 43 & 35 & 14 & 18 & 32 & 29 & 18 & 57 & 75 & 64 \\
\hline Great Basin & 2.0 & 14 & 16 & 7.9 & 11 & 10 & 22 & 19 & 13 & 25 & 38 & 27 \\
\hline South Pacific & 17 & 190 & 210 & 120 & 25 & 57 & 82 & 66 & 42 & 250 & 290 & 190 \\
\hline Pacific Northwest & 30 & 39 & 69 & 23 & 38 & 21 & 59 & 55 & 67 & 61 & 130 & 78 \\
\hline Hawaii & 2.0 & 6.0 & 8.0 & 6.4 & 1.9 & .5 & 2.4 & 1.9 & 3.9 & 6.5 & 10 & 8.3 \\
\hline Alaska & 1.2 & 4.9 & 6.1 & .3 & 0 & .1 & .1 & .1 & 1.2 & 5.0 & 6.2 & .4 \\
\hline $\begin{array}{l}\text { Puerto Rico and } \\
\text { Virgin Islands. }\end{array}$ & 8.7 & 1.6 & 10 & 9.2 & 3.2 & .8 & 3.9 & 3.6 & 12 & 2.4 & 14 & 13 \\
\hline $\begin{array}{l}\text { United States ex- } \\
\text { cluding Alaska, } \\
\text { Hawaii, Puerto } \\
\text { Rico and Virgin } \\
\text { Islands. }\end{array}$ & 140 & $1,900^{\circ}$ & 2,000 & 1,200 & 700 & 890 & 1,600 & 1,500 & 840 & 2,800 & 3,600 & 2,700 \\
\hline United States & 150 & 1,900 & 2,000 & 1,200 & 700 & 890 & 1,600 & 1,500 & 850 & 2,800 & 3,600 & 2,800 \\
\hline
\end{tabular}


domestic water were computed for this report using the following per capita rates:

\begin{tabular}{l|r|r}
\hline & $\begin{array}{r}\text { With } \\
\text { running } \\
\text { water } \\
\text { (gpd) }\end{array}$ & $\begin{array}{c}\text { Without } \\
\text { running } \\
\text { water } \\
\text { (gpd) }\end{array}$ \\
\hline Arizona. & 100 & 10 \\
California & 80 & 20 \\
Florida & 60 & 5 \\
Hawaii... & 160 & 70 \\
Idaho & 100 & 20 \\
Illinois & 60 & 10 \\
Kentucky. & 35 & 5 \\
New Jersey & 50 & 5 \\
New York & 60 & 10 \\
Puerto Rico & 75 & 10 \\
All other States. & 25 & 10 \\
\hline
\end{tabular}

The quantity of water used by livestock ranges widely, depending on kind and age of the animal and the temperature of the air (Sykes, 1955). Several authorities (Sykes. 1955; Marion, 1952; U.S. Inter-Agency Committee on the Arkansas-White-Red Basins, 1957) have given the water requirements of livestock. The quantity of water used by livestock in most States was computed using. the following rates per head:

\begin{tabular}{l|c}
\hline \multicolumn{1}{c|}{ Livestock } & $\begin{array}{c}\text { Use per } \\
\text { head (gpd) }\end{array}$ \\
\hline Milk cows. & 20 \\
Horses and mules. & 10 \\
Beef cattle & 10 \\
Sheeps & 3 \\
Goats... & 2 \\
Chickens. & 2 \\
Turkeys & .04 \\
\hline
\end{tabular}

Major exceptions to the above rates per head are as follows:

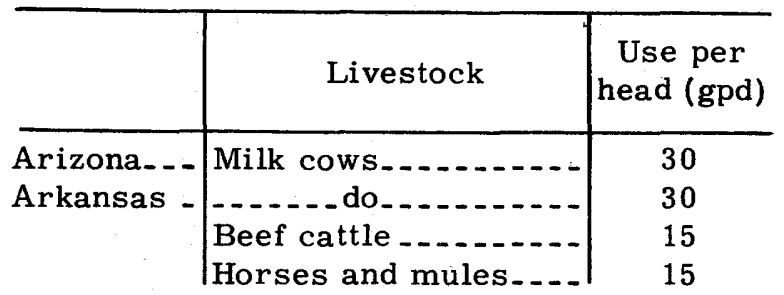

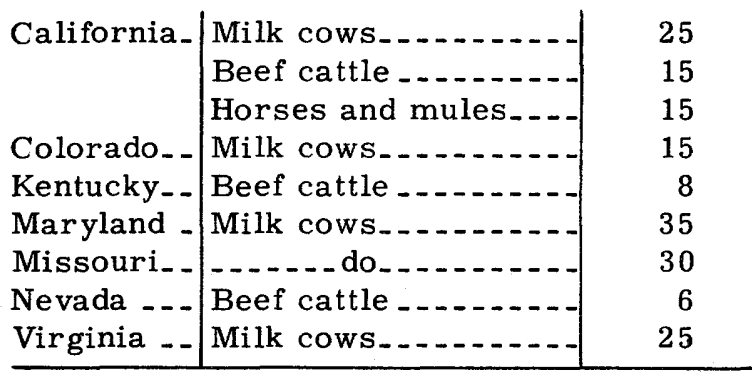

\section{IRRIGATION}

Water was withdrawn for irrigation in 1960 at the rate of 94 million acre-feet per year (tables 5 and 6 ) to irrigate 39 million acres. An additional 26 million acre-feet was lost in conveyance between the points of diversion and use. About two-thirds of the water was obtained from lakes, reservoirs, and streams; the remaining third was obtained from wells and springs.

Irrigation water is usually measured in acre-feet per year. However, in this report it is given also in average million gallons per day so that the quantities can be compared with and added to quantities of water used for other purposes. Irrigation water is applied during only a part of each year and at variable rates; therefore, the actual rate of application is much greater than the average daily rate given in tables 5 and 6.

Irrigation varies greatly throughout the Nation. Of the 39 million acres irrigated, almost 36 million acres was in the 17 Western States, whereas only 3.5 million acres was in the other 33 States and Puerto Rico. Therefore, the quantity of water used for irrigation in the Western States is much greater than in the remaining States and Puerto Rico. (See fig. 2). Furthermore, the water used for irrigation in the East is usually conveyed to the fields in pipes so the conveyance losses are very small compared to those losses in the Western States where the water is usually transported to the fields in ditches.

About 60 percent of the irrigation water applied in 1960 was consumed by evaporation and transpiration. This percentage is more than 25 times the percent consumed by selfsupplied industry, and about four times the percent consumed by public supplies. 
Table 5. - Water used for irrigation, by States, 1960

\begin{tabular}{|c|c|c|c|c|c|c|c|c|c|c|c|c|c|}
\hline & \multirow{2}{*}{$\begin{array}{c}\text { Acres } \\
\text { irrigated } \\
(1,000 \text { 's } \\
\text { of acres })\end{array}$} & \multicolumn{4}{|c|}{$\begin{array}{l}\text { Water delivered to farms }(1,000 \\
\text { ac-ft per year })\end{array}$} & \multirow{2}{*}{$\begin{array}{c}\text { Convey- } \\
\text { ance loss } \\
(1,000 \\
\mathrm{ac}-\mathrm{ft} / \mathrm{yr})\end{array}$} & \multirow{2}{*}{$\begin{array}{c}\text { Con- } \\
\text { sump- } \\
\text { tive use } \\
(1,000 \\
\mathrm{ac}-\mathrm{ft} / \mathrm{yr})\end{array}$} & \multicolumn{4}{|c|}{$\begin{array}{l}\text { Water delivered to farms (million } \\
\text { gallons per day) }\end{array}$} & \multirow{2}{*}{$\begin{array}{c}\text { Convey- } \\
\text { ance } \\
\text { loss } \\
\text { (mgd) }\end{array}$} & \multirow{2}{*}{$\begin{array}{c}\text { Con- } \\
\text { sump- } \\
\text { tive use } \\
\text { (mgd) }\end{array}$} \\
\hline & & $\begin{array}{l}\text { Surface } \\
\text { water }\end{array}$ & $\begin{array}{l}\text { Ground } \\
\text { water }\end{array}$ & $\begin{array}{l}\text { Other } \\
\text { water }\end{array}$ & $\begin{array}{l}\text { All } \\
\text { water }\end{array}$ & & & $\begin{array}{l}\text { Surface } \\
\text { water }\end{array}$ & $\begin{array}{l}\text { Ground } \\
\text { water }\end{array}$ & $\begin{array}{l}\text { Other } \\
\text { water }\end{array}$ & $\begin{array}{c}\text { All } \\
\text { water }\end{array}$ & & \\
\hline Alabama $\ldots . . . .$. & 27 & 11 & 2.7 & 0 & 14 & 0 & 14 & 9.8 & 2.4 & 0 & 12 & 0 & 12 \\
\hline Alaska........ & .36 & .03 & .06 & 0 & .09 & 0 & .06 & .03 & .05 & 0 & .08 & 0 & .05 \\
\hline Arizona $\ldots \ldots \ldots$ & 1,300 & 1,900 & 3,300 & 0 & 5,200 & 1,200 & 3,200 & 1,700 & 3,000 & 0 & 4,700 & 1,100 & 2,900 \\
\hline Arkansas _..... & 980 & 160 & 860 & 0 & 1,000 & 82 & $7 \cdot 10$ & 150 & 770 & 0 & 920 & 73 & 640 \\
\hline California_..... & 8,000 & 10,000 & 9,500 & 490 & 20,000 & 5,100 & 16,000 & 9,400 & 8,500 & 430 & 18,000 & 4,500 & 14,000 \\
\hline Colorado__._. & 3,200 & 8,000 & 2,100 & 43 & 10,000 & 1,000 & 5,400 & 7,100 & 1,800 & 38 & 9,000 & 930 & 4,800 \\
\hline Connecticut _...- & 5.5 & 1.1 & .06 & 0 & 1.2 & 0 & 1.2 & 1.0 & .05 & 0 & 1.0 & 0 & 1.0 \\
\hline Delaware $\ldots \ldots$. & 8.0 & 1.4 & 1.2 & 0 & 2.6 & 0 & 2.6 & 1.3 & 1.1 & 0 & 2.4 & 0 & 2.4 \\
\hline Florida & 680 & 440 & 300 & 0 & 740 & 22 & 740 & 390 & 270 & 0 & 660 & 20 & 660 \\
\hline Georgia_._. & 96 & 23 & 18 & 0 & 41 & 0 & 41 & 20 & 16 & 0 & 37 & 0 & 37 \\
\hline Hawaii_......... & 130 & 610 & 420 & 0 & 1,000 & 120 & 410 & 540 & 380 & 0 & 920 & 110 & 370 \\
\hline Idaho & 3,200 & 9,600 & 2,600 & 0 & 12,000 & 5,600 & 5,100 & 8,600 & 2,300 & 0 & 11,000 & 5,000 & 4,500 \\
\hline Illinois $\ldots \ldots \ldots$ & 11 & 1.4 & 1.2 & .05 & 2.6 & 0 & 2.6 & 1.2 & 1.1 & .04 & 2.4 & 0 & 2.4 \\
\hline Indiana $\ldots \ldots \ldots$ & 20 & 4.5 & 3.6 & 0 & 8.0 & 0 & 8.0 & 4.0 & 3.2 & 0 & 7.2 & 0 & 7.2 \\
\hline Iowa_-_-n_- & 79 & 26 & 42 & 0 & 68 & 0 & 68 & 23 & 38 & 0 & 61 & 0 & 61 \\
\hline Kansas _...... & 1,000 & 1,000 & 1,000 & 0 & 2,000 & 350 & 1,800 & 900 & 900 & 0 & 1,800 & 310 & 1,600 \\
\hline Kentucky__._. & 8.2 & 2.1 & .58 & .07 & 2.8 & 0 & 2.8 & 1.9 & .52 & .06 & 2.5 & 0 & 2.5 \\
\hline Louisiana & 510 & 530 & 380 & 0 & 910 & 270 & 640 & 470 & 340 & 0 & 810 & 240 & 570 \\
\hline Maine $\ldots \ldots \ldots$ & 3.0 & .98 & 0 & .01 & .99 & 0 & .99 & .88 & 0 & .01 & .88 & 0 & .88 \\
\hline Maryland ...... & 13 & 4.2 & 1.6 & .09 & 6.0 & 0 & 6.0 & 3.8 & 1.4 & .08 & 5.3 & 0 & 5.3 \\
\hline Massachusetts_- & 15 & 7.9 & .21 & .29 & 8.4 & 0 & 3.7 & 7.1 & .19 & .26 & 7.5 & 0 & 3.3 \\
\hline Michigan__._.-- & 68 & 16 & 8.7 & 0 & 25 & 0 & 25 & 14 & 7.8 & 0 & 22 & 0 & 22 \\
\hline Minnesota $\ldots \ldots$ & 20 & 3.8 & 4.2 & 0 & 8.0 & 0 & 8.0 & 3.4 & 3.8 & 0 & 7.1 & 0 & 7.1 \\
\hline Mississippi $\ldots \ldots$ & 310 & 210 & 360 & 0 & 570 & 10 & 460 & 190 & 320 & 0 & 510 & 9.2 & 410 \\
\hline Missouri__._. & 41 & 8.5 & 22 & 0 & 31 & 0 & 31 & 7.6 & 20 & 0 & 28 & 0 & 28 \\
\hline Montana $\ldots \ldots$ & 2,000 & 5,600 & 38 & 0 & 5,700 & 1,900 & 2,700 & 5,000 & 34 & 0 & 5,100 & 1,700 & 2,400 \\
\hline Nebraska _.....- & 2,600 & 1,000 & 1,500 & 0 & 2,500 & 1,300 & 1,200 & 900 & 1,300 & 0 & 2,200 & 1,200 & 1,100 \\
\hline Nevada $\ldots . . . . .-$ & 660 & 1,700 & 300 & 1.1 & 2,000 & 430 & 1,100 & 1,500 & 270 & .97 & 1,700 & 380 & 960 \\
\hline New Hampshire. & 3.6 & 1.1 & .18 & .24 & 1.5 & 0 & 1.4 & .97 & .16 & .21 & 1.4 & 0 & 1.3 \\
\hline New Jersey _..- & 66 & 11 & 29 & 0 & 40 & 0 & 40 & 9.5 & 26 & 0 & 35 & 0 & 35 \\
\hline
\end{tabular}




\begin{tabular}{|c|c|c|c|c|c|c|c|c|c|c|c|c|c|}
\hline New Mexico $\ldots . .$. & 930 & 1,000 & 1,000 & 10 & 2,100 & 640 & 1,500 & 920 & 910 & 9.1 & 1,800 & 570 & 1,300 \\
\hline New York & 57 & 20 & 11 & 0 & 31 & 0 & 31 & 18 & 9.5 & 0 & 28 & 0 & 28 \\
\hline North Carolina & 60 & 23 & 7.5 & 0 & 30 & 0 & 30 & 20 & 6.7 & 0 & 27 & 0 & 27 \\
\hline North Dakota_. & 62 & 94 & .14 & 0 & 94 & 31 & 63 & 84 & .13 & 0 & 84 & 28 & 56 \\
\hline Ohio & 21 & 6.6 & 2.9 & 0 & 9.5 & 0 & 8.6 & 5.9 & 2.6 & 0 & 8.5 & 0 & 7.7 \\
\hline Oklahoma_..... & 310 & 94 & 210 & 0 & 300 & 18 & 210 & 84 & 190 & 0 & 270 & 16 & 190 \\
\hline Oregon & 1,600 & 5,100 & 270 & 0 & 5,400 & 1,700 & 3,100 & 4,600 & 240 & 0 & 4,800 & 1,500 & 2,800 \\
\hline Pennsylvania_._- & 17 & 3.0 & .28 & 0 & 3.3 & 0 & 3.3 & 2.7 & .25 & 0 & 3.0 & 0 & 3.0 \\
\hline Puerto Rico & 100 & 130 & 190 & 0 & 320 & 38 & 280 & 110 & 170 & 0 & 280 & 34 & 250 \\
\hline Rhode Island & .50 & .19 & .05 & .01 & .25 & 0 & .25 & .17 & .04 & .01 & .22 & 0 & .22 \\
\hline South Carolina & 68 & 30 & 21 & 0 & 51 & 0 & 51 & 27 & 19 & 0 & 46 & 0 & 46 \\
\hline South Dakota & 140 & 130 & 38 & 0 & 170 & 97 & 130 & 120 & 34 & 0 & 150 & 86 & 110 \\
\hline Tennessee _..... & 26 & 11 & 2.0 & 0 & 13 & 0 & 13 & 9.7 & 1.8 & 0 & 11 & 0 & 11 \\
\hline Texas & 7,100 & 1,300 & 8,600 & 37 & 9,900 & 2,600 & 7,000 & 1,200 & 7,700 & 33 & 8,900 & 2,300 & 6,200 \\
\hline Utah $\ldots$ & 1,200 & 3,400 & 270 & 54 & 3,700 & 840 & 2,500 & 3,000 & 240 & 48 & 3,300 & 750 & 2,200 \\
\hline Vermont_... & 2.0 & .81 & .03 & 0 & .84 & 0 & .80 & .72 & .03 & 0 & .75 & 0 & .71 \\
\hline Virginia _.... & 40 & 26 & 13 & .80 & & 0 & 39 & 23 & 12 & .71 & 36 & 0 & 35 \\
\hline Virgin Islands & .10 & .36 & .04 & 0 & .40 & 0 & .36 & .32 & .04 & 0 & .36 & 0 & .32 \\
\hline Washington $\ldots$ & 1,000 & 3,700 & 470 & 0 & 4,100 & 1,100 & 1,500 & 3,300 & 420 & 0 & 3,700 & 1,000 & 1,300 \\
\hline West Virginia & 2.1 & 1.3 & .07 & .04 & 1.4 & 0 & 1.4 & 1.1 & .06 & .04 & 1.2 & & 1.2 \\
\hline Wisconsin $\ldots$ & 41 & 3.7 & 14 & .79 & 18 & 0 & 18 & 3.3 & 12 & .71 & 16 & 0 & 16 \\
\hline Wyoming . . . & 1,400 & 3,400 & 64 & 0 & 3,500 & 1,400 & 2,100 & 3,100 & 57 & 0 & 3,100 & 1,300 & 1,900 \\
\hline $\begin{array}{l}\text { District of } \\
\text { Columbia. }\end{array}$ & 0 & 0 & 0 & 0 & 0 & 0 & 0 & 0 & 0 & 0 & 0 & 0 & 0 \\
\hline $\begin{array}{l}\text { United States ex- } \\
\text { cluding Alaska, } \\
\text { Hawaii, Puerto } \\
\text { Rico and Virgin } \\
\text { Islands. }\end{array}$ & 39,000 & 59,000 & 33,000 & 630 & 93,000 & 26,000 & 57,000 & 53,000 & 30,000 & 570 & 83,000 & 23,000 & 51,000 \\
\hline United States_. & 39,000 & 60,000 & 34,000 & 630 & 94,000 & 26,000 & 58,000 & 53,000 & 30,000 & 570 & 84,000 & 23,000 & 52,000 \\
\hline
\end{tabular}


Table 6. -Water used for irrigation, by reaions, 1960

\begin{tabular}{|c|c|c|c|c|c|c|c|c|c|c|c|c|c|}
\hline & \multirow{2}{*}{$\begin{array}{c}\text { Acres } \\
\text { irrigated } \\
(1,000 \text { 's } \\
\text { of acres) }\end{array}$} & \multicolumn{4}{|c|}{$\begin{array}{l}\text { Water delivered to farms }(1,000 \\
\text { ac-ft per year })\end{array}$} & \multirow{2}{*}{$\begin{array}{c}\text { Convey- } \\
\text { ance loss } \\
(1,000 \\
\mathrm{ac}-\mathrm{ft} / \mathrm{yr})\end{array}$} & \multirow{2}{*}{$\begin{array}{c}\text { Con- } \\
\text { sump- } \\
\text { tive use } \\
(1,000 \\
\text { ac-ft/yr })\end{array}$} & \multicolumn{4}{|c|}{$\begin{array}{l}\text { Water delivered to farms (million } \\
\text { gallons per day) }\end{array}$} & \multirow{2}{*}{$\begin{array}{c}\text { Convey- } \\
\text { ance } \\
\text { loss } \\
\text { (mgd) }\end{array}$} & \multirow{2}{*}{$\begin{array}{l}\text { Con- } \\
\text { sump- } \\
\text { tive use } \\
\text { (mgd) }\end{array}$} \\
\hline & & $\begin{array}{l}\text { Surface } \\
\text { water }\end{array}$ & $\begin{array}{l}\text { Ground } \\
\text { water }\end{array}$ & $\begin{array}{l}\text { Other } \\
\text { water }\end{array}$ & $\begin{array}{c}\text { All } \\
\text { water }\end{array}$ & & & $\begin{array}{l}\text { Surface } \\
\text { water }\end{array}$ & $\begin{array}{l}\text { Ground } \\
\text { water }\end{array}$ & $\begin{array}{l}\text { Other } \\
\text { water }\end{array}$ & $\begin{array}{c}\text { All } \\
\text { water }\end{array}$ & & \\
\hline New England ... & 28 & 11 & 0.51 & 0.55 & 13 & 0 & 7.8 & 10 & 0.46 & 0.49 & 11 & 0 & 6.9 \\
\hline $\begin{array}{l}\text { Delaware- } \\
\text { Hudson. }\end{array}$ & 120 & 17 & 40 & 0 & 57 & 0 & 57 & 15 & 36 & 0 & 51 & 0 & 51 \\
\hline Chesapeake _. _- & 44 & 21 & 14 & .58 & 35 & 0 & 34 & 18 & 12 & .52 & 31 & 0 & 31 \\
\hline South Atlantic _- & 840 & 510 & 320 & .30 & 830 & 22 & 830 & 450 & 290 & .28 & 740 & 19 & 740 \\
\hline Eastern Gulf _.. & 110 & 29 & 35 & 0 & 64 & .67 & 64 & 26 & 32 & 0 & 57 & .60 & 57 \\
\hline $\begin{array}{l}\text { Tennessee- } \\
\text { Cumberland. }\end{array}$ & 30 & 13 & 1.7 & .01 & 15 & 0 & 15 & 12 & 1.5 & .01 & 14 & 0 & 14 \\
\hline Ohio ....... & 33 & 10 & 3.7 & .11 & 14 & 0 & 14 & 9.4 & 3.3 & .10 & 13 & 0 & 12 \\
\hline $\begin{array}{l}\text { Eastern Great } \\
\text { Lakes St. } \\
\text { Lawrence. }\end{array}$ & 34 & 19 & 1.6 & 0 & 21 & 0 & 21 & 17 & 1.5 & 0 & 19 & 0 & 18 \\
\hline $\begin{array}{l}\text { Western Great } \\
\text { Lakes. }\end{array}$ & 79 & 16 & 14 & .33 & 30 & 0 & 30 & 14 & 13 & .29 & 27 & 0 & 27 \\
\hline Hudson Bay .... & 12 & 15 & .16 & 0 & 15 & 4.8 & 10 & 13 & .14 & 0 & 13 & 4.3 & 9.0 \\
\hline $\begin{array}{l}\text { Upper } \\
\text { Mississippi. }\end{array}$ & 92 & 18 & 30 & .51 & 49 & 0 & 49 & 16 & 27 & .46 & 44 & 0 & 44 \\
\hline Upper Missouri & 7,000 & 12,000 & 2,500 & 26 & 14,000 & 4,900 & 7,700 & 11,000 & 2,200 & 24 & 13,000 & 4,400 & 6,900 \\
\hline $\begin{array}{l}\text { Lower } \\
\text { Missouri. }\end{array}$ & 61 & 22 & 30 & 0 & 51 & .39 & 51 & 19 & 26 & 0 & 46 & . .35 & 46 \\
\hline $\begin{array}{l}\text { Lower } \\
\text { Mississippi. }\end{array}$ & 650 & 290 & 660 & 0 & 950 & 57 & 730 & 260 & 590 & 0 & 850 & 50 & 660 \\
\hline $\begin{array}{l}\text { Upper } \\
\text { Arkansas-Red. }\end{array}$ & 2,300 & 2,500 & 1,900 & 17 & 4,400 & 640 & 3,300 & 2,200 & 1,700 & 15 & 3,900 & 570 & 2,900 \\
\hline $\begin{array}{l}\text { Lower } \\
\quad \text { Arkansas-Red } \\
\text { White. }\end{array}$ & 760 & 150 & 630 & 0 & 780 & 62 & 550 & 140 & 560 & 0 & 700 & 55 & 490 \\
\hline Western Gulf._- & 8,300 & 3,500 & 10,000 & 47 & 14,000 & 3,300 & 9,200 & 3,100 & 9,300 & 42 & 12,000 & 3,000 & 8,200 \\
\hline Colorado _._._. & 3,100 & 8,900 & 3,600 & 2.1 & 12,000 & 2,700 & 7,700 & 7,900 & 3,200 & 1.8 & 11,000 & 2,400 & 6,900 \\
\hline Great Basin & 2,100 & 5,000 & 710 & 53 & 5,800 & 1,400 & 3,600 & 4,500 & 640 & 48 & 5,100 & 1,200 & 3,200 \\
\hline South Pacific & 7,700 & 8,700 & 9,200 & 490 & 18,000 & 4,400 & 14,000 & 7,800 & 8,200 & 430 & 16,000 & 4,000 & 13,000 \\
\hline
\end{tabular}




\begin{tabular}{|c|c|c|c|}
\hline $\begin{array}{l}8 \\
8 \\
\infty\end{array}$ & $\begin{array}{ll}\circ & 0 \\
\text { N } & \text { N } \\
\text { N }\end{array}$ & $\begin{array}{l}8 \\
8 \\
\text { in }\end{array}$ & $\begin{array}{l}8 \\
8 \\
\text { in } \\
\text { in }\end{array}$ \\
\hline $\begin{array}{l}\circ \\
\text { 尺 } \\
\text { n- }\end{array}$ & $\underset{-1}{\circ} \bigcirc \underset{m}{H}$ & $\begin{array}{l}\text { O } \\
\text { ○ } \\
\text { N }\end{array}$ & $\begin{array}{l}8 \\
8 \\
\text { ก } \\
\text { N }\end{array}$ \\
\hline $\begin{array}{l}8 \\
8 \\
0 \\
9 \\
-1\end{array}$ & 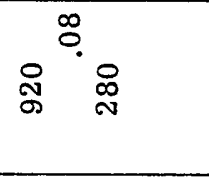 & $\begin{array}{l}8 \\
8 \\
\infty \\
\infty\end{array}$ & $\begin{array}{l}8 \\
8 \\
0 \\
\infty\end{array}$ \\
\hline 0 & 000 & $\underset{10}{\stackrel{0}{10}}$ & $\underset{0}{2}$ \\
\hline $\begin{array}{l}\text { ○. } \\
\text { ণ } \\
\text { ஸे }\end{array}$ & 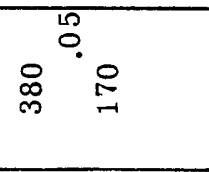 & $\begin{array}{l}8 \\
\circ \\
\circ \\
\circ\end{array}$ & $\begin{array}{l}8 \\
8 \\
0 \\
0\end{array}$ \\
\hline $\begin{array}{l}8 \\
8 \\
0 \\
0 \\
1\end{array}$ & 윰요 & $\begin{array}{l}8 \\
8 \\
10 \\
10\end{array}$ & $\begin{array}{l}8 \\
8 \\
0 \\
10 \\
\end{array}$ \\
\hline $\begin{array}{l}\circ \\
8 \\
\infty\end{array}$ & 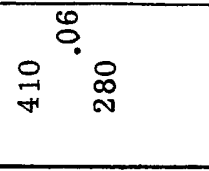 & $\begin{array}{l}8 \\
0 \\
5 \\
10\end{array}$ & $\begin{array}{l}8 \\
8 \\
00^{\circ} \\
10\end{array}$ \\
\hline & 옥 0 m & ஜ & $\begin{array}{l}8 \\
0 \\
0 \\
0\end{array}$ \\
\hline $\begin{array}{l}8 \\
\stackrel{-}{\text { N }}\end{array}$ & 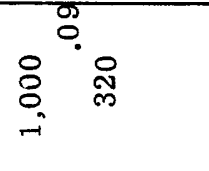 & $\begin{array}{l}\circ \\
\text { व } \\
\text { ले }\end{array}$ & \& \\
\hline & 000 & $\begin{array}{l}\text { ㅇ } \\
0 \\
\end{array}$ & $\begin{array}{l}0 \\
\text { ల్ } \\
0\end{array}$ \\
\hline $\begin{array}{l}8 \\
\text { ळ. } \\
\text { m. }\end{array}$ & 궁 $\stackrel{0}{\circ}$ & $\begin{array}{l}\stackrel{8}{\circ} \\
\text { ల్ల }\end{array}$ & $\begin{array}{l}8 \\
8 \\
\end{array}$ \\
\hline $\begin{array}{l}8 \\
8 \\
0 \\
\infty \\
-1 \\
\end{array}$ & 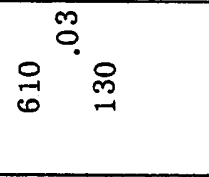 & $\begin{array}{l}8 \\
8 \\
\circ \\
\text { in }\end{array}$ & $\begin{array}{l}8 \\
8 \\
8\end{array}$ \\
\hline $\begin{array}{l}8 \\
\stackrel{8}{10}\end{array}$ & 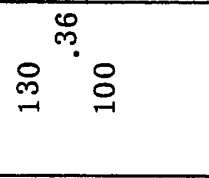 & $\begin{array}{l}\circ \\
8 \\
\rho^{\circ}\end{array}$ & $\begin{array}{l}\text { : } \\
\text { ᄋ. } \\
\text { ळ }\end{array}$ \\
\hline 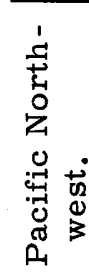 & 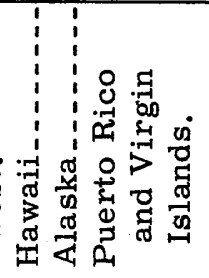 & 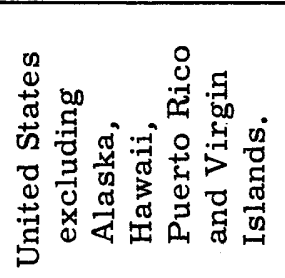 & 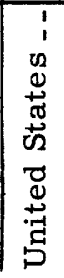 \\
\hline
\end{tabular}

SELF-SUPPLIED INDUSTRIAL USE

Industry used an average of $140,000 \mathrm{mgd}$ of self-supplied water during 1960, including $100,000 \mathrm{mgd}$ used for fuel-electric power (tables 7 and 8 ). About 95 percent of the selfsupplied industrial water was obtained from surface sources. In contrast to water used for irrigation, most industrial water is used east of the Mississippi River (fig. 3), and only about 2 percent of the industrial water withdrawn is consumed.

Water has many industrial uses, including processing, cooling, washing, conveyance of material, boiler feed, and sanitation. The commercial fish growing (fish farms and minnow farms or bait hatcheries) is an unusual industry. This industry used $47 \mathrm{mgd}$ in 1960 in Arkansas. About 22 mgd or almost half this water was consumed. Fish hatcheries and log ponds in Oregon withdrew slightly more than $600 \mathrm{mgd}$, of which only $16 \mathrm{mgd}$ was consumed. In Oregon these uses require a water right. Some industries require water containing small amounts of dissolved solids, whereas other industries are relatively unconcerned about the dissolved-solids content of the water. Most industrial water is self supplied, although a small amount is purchased from public supplies (tables 1 and 2 ).

About 94 percent of the self-supplied in-dustrial water is used for cooling (fig. 4). Most water for cooling is returned to a stream or an aquifer unchanged except for an increase in temperature. Cooling water need not have a low dissolved-solids content; some cooling equipment is designed for use of sea water or other saline water. Almost onefourth of the water withdrawn by industry was saline.

FUEL-ELECTRIC POWER (PUBLIC UTILITY)

The amount of water used by public utilities for fuel-electric power was more than twice the amount of self-supplied industrial water used by other industries (tables 7 and 8). Almost all the water used by public utilities for generation of fuel-electric power was for condenser cooling (tables 9 and 10). Water for other uses, such as boiler feed, sanitary services, cooling of machinery within the plant, and irrigation of lawns was a little more than 1 percent of the total. All water for these uses was fresh. 
$\stackrel{\infty}{\infty}$

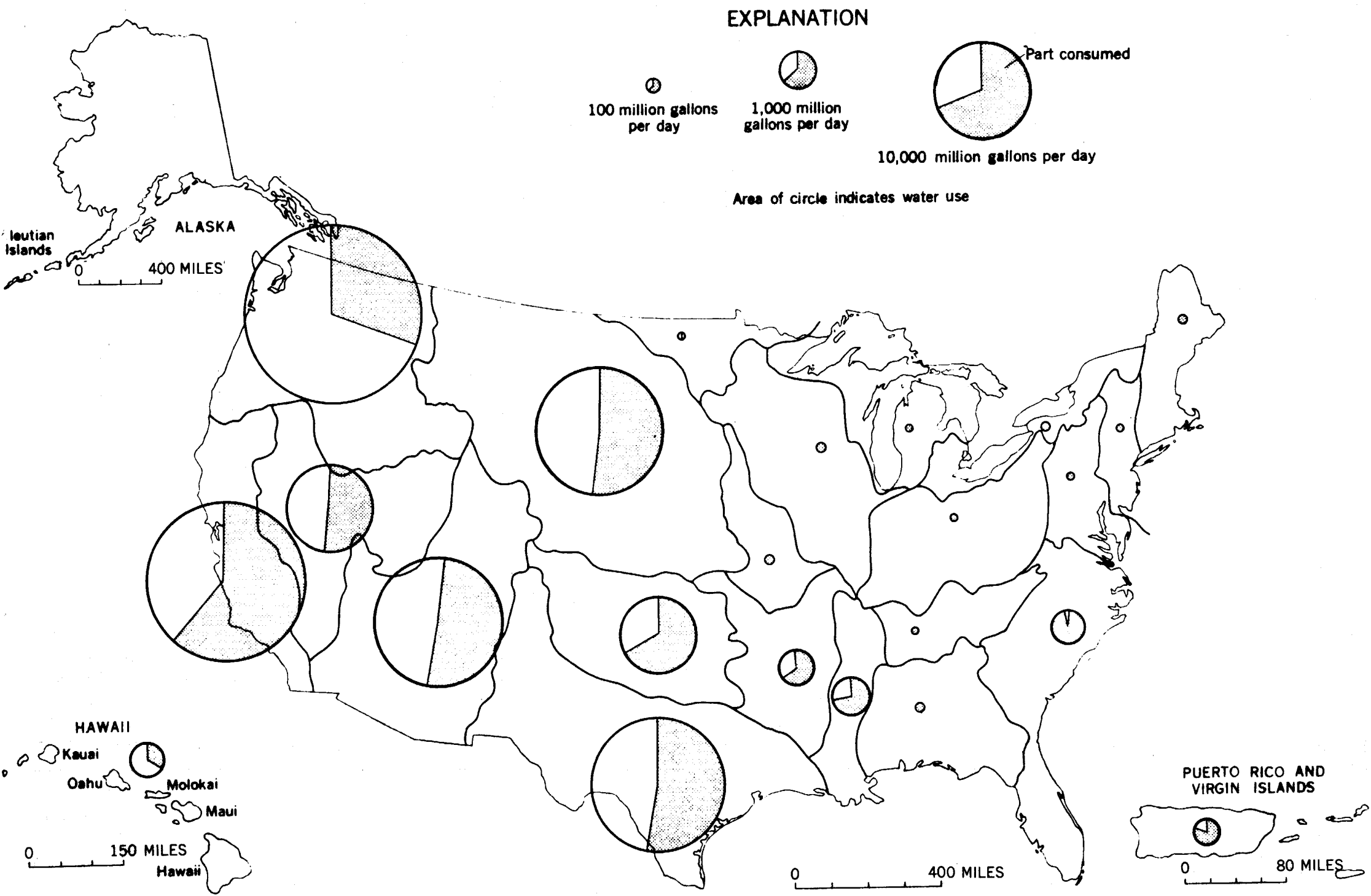

Figure 2. - Water used for irrigation (including conveyance losses), by regions in the United States, 1960. 
Table 7.-Self-supplied indus-

[Million gal-

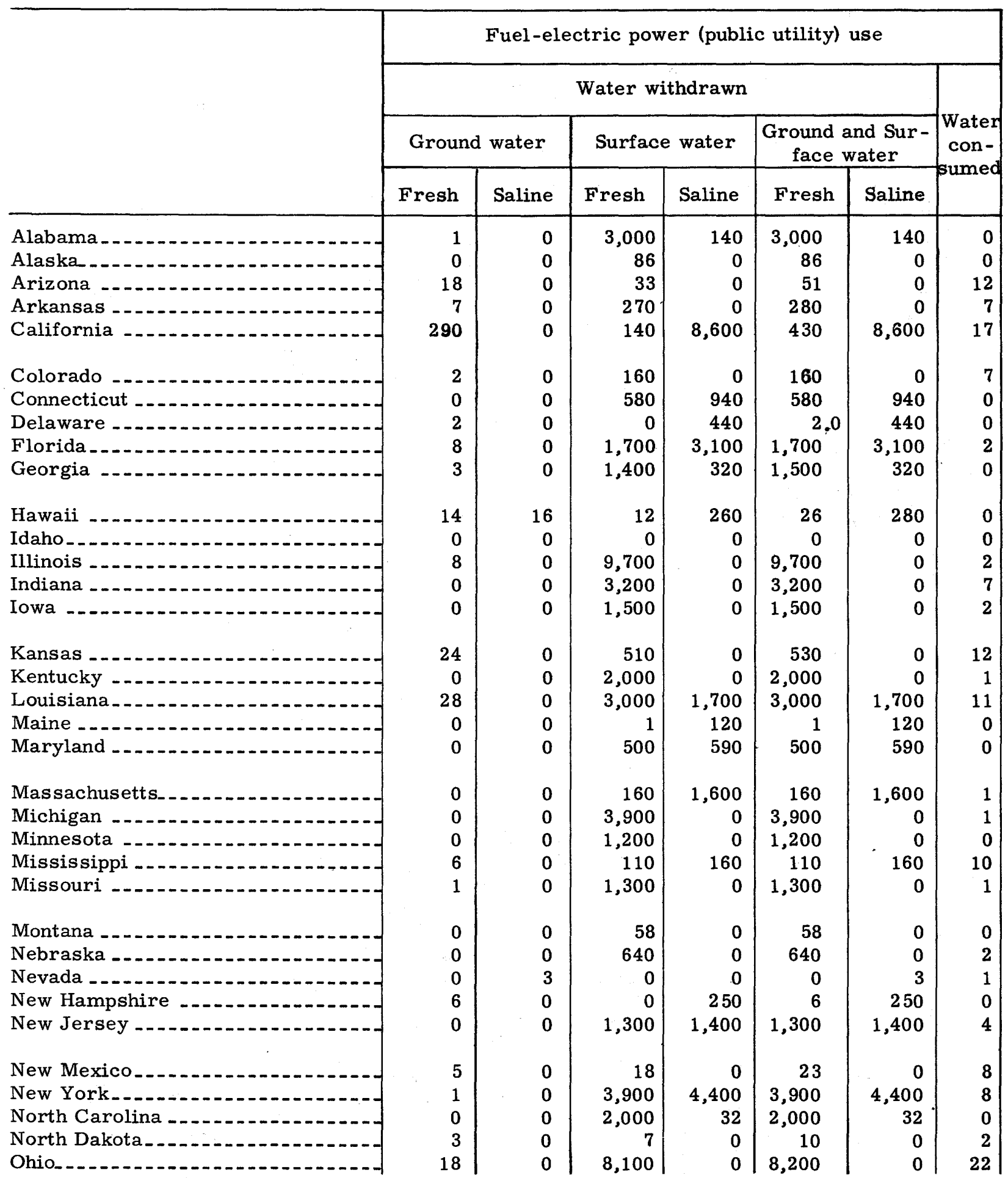


trial water, by States, 1960

lons per day]

\begin{tabular}{|c|c|c|c|c|c|c|c|c|c|c|c|c|}
\hline \multicolumn{8}{|c|}{ Other uses } & \multicolumn{5}{|c|}{ All industrial uses } \\
\hline \multicolumn{7}{|c|}{ Water withdrawn } & \multirow{3}{*}{$\begin{array}{c}\text { Water } \\
\text { con- } \\
\text { sumed }\end{array}$} & \multicolumn{4}{|c|}{ Water withdrawn } & \multirow{3}{*}{$\begin{array}{l}\text { Water } \\
\text { con- } \\
\text { sumed }\end{array}$} \\
\hline \multicolumn{2}{|c|}{ Ground water } & \multicolumn{2}{|c|}{ Surface water } & \multirow{2}{*}{$\begin{array}{l}\text { Sew } \\
\text { age }\end{array}$} & \multicolumn{2}{|c|}{ All water } & & & & & & \\
\hline Fresh & Saline & Fresh & Saline & & Fresh & Saline & & & & age & & \\
\hline 79 & 0 & 810 & 0 & 0 & 890 & 0 & 87 & 3,900 & 140 & 0 & 4,000 & 87 \\
\hline 12 & 0 & 70 & 0 & 0 & 82 & 0 & 0 & 170 & 0 & 0 & 170 & 0 \\
\hline 62 & 0 & 16 & 0 & 0 & 78 & 0 & 24 & 130 & 0 & 0 & 130 & 36 \\
\hline 140 & 0 & 43 & 0 & 0 & 190 & 0 & 40 & 470 & 0 & 0 & 470 & 47 \\
\hline 310 & 140 & 42 & 510 & .5 & 350 & 650 & 81 & 790 & 9,300 & .5 & 10,000 & 98 \\
\hline 35 & 10 & 120 & 10 & 0 & 160 & 20 & 36 & 310 & 20 & 0 & 330 & 44 \\
\hline 40 & 0 & 210 & 66 & 0 & 250 & 66 & 20 & 830 & 1,000 & 0 & 1,800 & 20 \\
\hline 27 & 0 & 28 & 380 & 0 & 55 & 380 & 54 & 57 & 820 & 0 & 880 & 54 \\
\hline 680 & 0 & 79 & 260 & 0 & 760 & 260 & 300 & 2,500 & 3,300 & 0 & 5,800 & 300 \\
\hline 230 & 0 & 76 & 100 & 0 & 310 & 100 & 6.9 & 1,800 & 430 & 0 & 2,200 & 7 \\
\hline 110 & 5.0 & 33 & .1 & 0 & 140 & 5.1 & 13 & 170 & 280 & 0 & 450 & 13 \\
\hline 91 & 0 & 90 & 0 & 0 & 180 & 0 & 36 & 180 & 0 & 0 & 180 & 36 \\
\hline 280 & 40 & 1,800 & 0 & 0 & 2,100 & 40 & 44 & 12,000 & 40 & 0 & 12,000 & 46 \\
\hline 150 & 11 & 1,900 & 0 & 0 & 2,000 & 11 & 78 & 5,300 & 11 & 0 & 5,300 & 85 \\
\hline 74 & 0 & 37 & 0 & 0 & 110 & 0 & 11 & 1,600 & 0 & 0 & 1,600 & 13 \\
\hline 120 & 0 & 60 & 0 & 0 & 180 & 0 & 8.9 & 710 & 0 & 0 & 710 & 21 \\
\hline 68 & .6 & 180 & .3 & 0 & 250 & .9 & 24 & 2,300 & 1 & 0 & 2,300 & 25 \\
\hline 310 & 39 & 1,700 & 0 & 0 & 2,100 & 39 & 540 & 5,000 & 1,700 & 0 & 6,800 & 550 \\
\hline 12 & 0 & 340 & 3.0 & 0 & 350 & 3.0 & 25 & 350 & 120 & 0 & 480 & 25 \\
\hline 49 & 0 & 140 & 690 & 70 & 190 & 690 & 74 & 700 & 1,300 & 70 & 2,000 & 74 \\
\hline 66 & 0 & 380 & 140 & 0 & 440 & 140 & 30 & 600 & 1,800 & 0 & 2,300 & 31 \\
\hline 99 & 14 & 1,700 & 0 & 0 & 1,800 & 14 & 51 & 5,800 & 14 & 0 & 5,800 & 52 \\
\hline 120 & 0 & 720 & 0 & 0 & 840 & 0 & 59 & 2,100 & 0 & 0 & 2,100 & 59 \\
\hline 160 & 0 & 58 & 0 & 0 & 220 & 0 & 29 & 330 & 160 & 0 & 480 & 39 \\
\hline 55 & 3.0 & 110 & 0 & 0 & 160 & 3.0 & 9.0 & 1,500 & 3 & 0 & 1,500 & 10 \\
\hline 35 & 1.0 & 170 & 0 & 0 & 200 & 1.0 & 23 & 260 & 1 & 0 & 260 & 23 \\
\hline 12 & 0 & 32 & 0 & 0 & 44 & 0 & 4.2 & 680 & 0 & 0 & 680 & 6 \\
\hline 34 & 0 & 10 & 0 & .2 & 44 & 0 & 8.8 & 44 & 3 & .2 & 48 & 10 \\
\hline 2.0 & 0 & 150 & 0 & 0 & 150 & 0 & 8.0 & 160 & 250 & 0 & 410 & 8 \\
\hline 190 & 7.6 & 370 & 760 & 0 & 560 & 760 & 150 & 1,800 & 2,100 & 0 & 4,000 & 150 \\
\hline 18 & .1 & 4.1 & 0 & 0 & 22 & .1 & 4.5 & 45 & & 0 & 45 & 12 \\
\hline 150 & 15 & 1,600 & 1,300 & 0 & 1,700 & 1,300 & 120 & 5,600 & 5,800 & 0 & 11,000 & 130 \\
\hline 33 & 0 & 230 & 0 & 0 & 260 & 0 & 22 & 2,300 & 32 & 0 & 2,300 & 22 \\
\hline 1.6 & 6.0 & 1.4 & 0 & 0 & 3.0 & 6.0 & .9 & 13 & 6 & 0 & 19 & 2.9 \\
\hline 270 & 0 & 2,300 & 0 & 0 & 2,600 & 0 & 87 & 12,000 & 0 & 0 & 12,000 & 110 \\
\hline
\end{tabular}


Table 7. - Self-supplied industrial

\begin{tabular}{|c|c|c|c|c|c|c|c|}
\hline \multirow{4}{*}{$\theta$} & \multicolumn{7}{|c|}{ Fuel-electric power (public utility) use } \\
\hline & \multicolumn{6}{|c|}{ Water withdrawn } & \multirow{3}{*}{$\begin{array}{l}\text { Water } \\
\text { con- } \\
\text { sumec }\end{array}$} \\
\hline & \multicolumn{2}{|c|}{ Ground water } & \multicolumn{2}{|c|}{ Surface water } & \multicolumn{2}{|c|}{$\begin{array}{l}\text { Ground and Sur - } \\
\text { face water }\end{array}$} & \\
\hline & Fresh & Saline & Fresh & Saline & Fresh & Saline & \\
\hline Oklahoma & 6 & 0 & 180 & 120 & 190 & 120 & 8 \\
\hline Oregon & 0 & 0 & 7 & 0 & 7 & 0 & 0 \\
\hline Pennsylvania _.. & 0 & 0 & 6,600 & 0 & 6,600 & 0 & 4 \\
\hline Puerto Rico and Virgin Islands _- & 0 & 0 & 4 & 490 & 4 & 490 & 0 \\
\hline Rhode Island & 0 & 0 & 0 & 300 & 0 & 300 & 0 \\
\hline South Carolina & 0 & 0 & 560 & 95 & 560 & 95 & 2 \\
\hline South Dakota & 0 & 0 & 1 & 0 & 1 & 0 & 1 \\
\hline Tennessee & 0 & 0 & 3,900 & 0 & 3,900 & 0 & 1 \\
\hline Texas & 470 & 0 & 2,000 & 1,300 & 2,500 & 1,300 & 52 \\
\hline Utah & 0 & 0 & 77 & 0 & 77 & 0 & 3 \\
\hline Vermont & 0 & 0 & 29 & 0 & 29 & 0 & 0 \\
\hline Virginia & 0 & 0 & 2,500 & 810 & 2,500 & 810 & 2 \\
\hline Washington & 0 & 0 & 0 & 0 & 0 & 0 & 0 \\
\hline West Virginia & 0 & 0 & 2,200 & 0 & 2,200 & 0 & 10 \\
\hline Wisconsin & 0 & 0 & 2,900 & 0 & 2,900 & 0 & 0 \\
\hline Wyoming & 0 & 0 & 84 & 0 & 84 & 0 & 1 \\
\hline District of Columbia & 0 & 0 & 270 & 0 & 270 & 0 & 0 \\
\hline $\begin{array}{l}\text { United States excluding Alaska, } \\
\text { Hawaii, Puerto Rico and Virgin } \\
\text { Islands. }\end{array}$ & 910 & 3 & 72,000 & 26,000 & 73,000 & 26,000 & 220 \\
\hline United States & 920 & 19 & 72,000 & 27,000 & 73,000 & 27,000 & 220 \\
\hline
\end{tabular}

Table 8. - Self-supplied indus-

[Million gal-

\begin{tabular}{|c|c|c|c|c|c|c|c|c|}
\hline \multirow{4}{*}{ 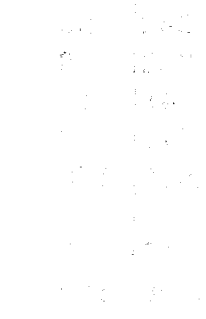 } & \multirow{4}{*}{ Region } & \multicolumn{7}{|c|}{ Fuel-electric power (public utility) use } \\
\hline & & \multicolumn{6}{|c|}{ Water withdrawn } & \multirow{3}{*}{$\begin{array}{c}\text { Water } \\
\text { con- } \\
\text { sumed }\end{array}$} \\
\hline & & \multicolumn{2}{|c|}{ Ground water } & \multicolumn{2}{|c|}{ Surface water } & \multicolumn{2}{|c|}{$\begin{array}{c}\text { Ground and sur- } \\
\text { face water }\end{array}$} & \\
\hline & & Fresh & Saline & Fresh & Saline & Fresh & Saline & \\
\hline \multirow{5}{*}{\multicolumn{2}{|c|}{$\begin{array}{l}\text { New England } \\
\text { Delaware-Hudson } \\
\text { Chesapeake } \\
\text { South Atlantic } \\
\text { Eastern Gulf }\end{array}$}} & 6 & 0 & 620 & 3,200 & 630 & 3,200 & 1 \\
\hline & & 3 & 0 & 5,200 & 6,100 & 5,200 & 6,100 & 13 \\
\hline & & 0 & 0 & 2,900 & 1,200 & 2,900 & 1,200 & 2 \\
\hline & & 10 & 0 & 5,200 & 3,600 & 5,200 & 3,600 & 3 \\
\hline & & 7 & 0 & 3,200 & 610 & 3,200 & 610 & \\
\hline
\end{tabular}


water, by States, 1960-Continued

\begin{tabular}{|c|c|c|c|c|c|c|c|c|c|c|c|c|}
\hline \multicolumn{8}{|c|}{ Other uses } & \multicolumn{5}{|c|}{ All industrial uses } \\
\hline \multicolumn{7}{|c|}{ Water withdrawn } & \multirow{3}{*}{$\begin{array}{c}\text { Water } \\
\text { con- } \\
\text { sumed }\end{array}$} & \multicolumn{4}{|c|}{ Water withdrawn } & \multirow{3}{*}{$\begin{array}{c}\text { Water } \\
\text { con- } \\
\text { sumed }\end{array}$} \\
\hline Ground & water & \multicolumn{2}{|c|}{ Surface water } & \multirow{2}{*}{$\begin{array}{l}\text { Sew - } \\
\text { age }\end{array}$} & \multicolumn{2}{|c|}{ All water } & & Frech & Soline & Sew - & All & \\
\hline Fresh & Saline & Fresh & Saline & & Fresh & Saline & & & & & & \\
\hline 23 & 46 & 26 & 11 & 0 & 50 & 57 & 21 & 240 & 180 & 0 & 420 & 29 \\
\hline 140 & $\mathbf{0}$ & 1,000 & 0 & 0 & 1,200 & 0 & 33 & 1,200 & 0 & 0 & 1,200 & 33 \\
\hline 300 & 0 & 4,100 & 460 & 0 & 4,400 & 460 & 190 & 11,000 & 460 & 0 & 11,000 & 200 \\
\hline 29 & 1.6 & 130 & 160 & 0 & 160 & 160 & 6.9 & 160 & 650 & 0 & 810 & 6.9 \\
\hline 15 & 0 & 32 & .3 & 0 & 47 & .3 & 2.2 & 47 & 300 & 0 & 340 & 2.2 \\
\hline 54 & 0 & 84 & 28 & 0 & 140 & 28 & 13 & 700 & 120 & 0 & 820 & 15 \\
\hline 6.7 & 3.9 & 5.8 & 0 & 0 & 12 & 3.9 & 4.4 & 14 & 3.9 & 0 & 17 & 5.4 \\
\hline 420 & 0 & 940 & 0 & 0 & 1,400 & 0 & 310 & 5,200 & 0 & 0 & 5,200 & 310 \\
\hline 330 & 11 & 430 & 3.6 & 0 & 760 & 15 & 100 & 3,300 & 1,400 & 0 & 4,600 & 160 \\
\hline 58 & 3.0 & 150 & 5.5 & 0 & 210 & 8.5 & 4.6 & 290 & 8.5 & 0 & 300 & 7.6 \\
\hline 9.1 & 0 & 25 & 0 & 0 & 34 & 0 & 2.0 & 63 & 0 & 0 & 63 & 2.0 \\
\hline 51 & 0 & 1,100 & 85 & 0 & 1,200 & 85 & 0 & 3,700 & 900 & 0 & 4,600 & 2.0 \\
\hline 170 & 0 & 520 & 48 & 0 & 690 & 48 & 14 & 690 & 48 & 0 & 740 & 14 \\
\hline 73 & .4 & 2,200 & 0 & 0 & 2,300 & .4 & 120 & 4,500 & .4 & 0 & 4,500 & 130 \\
\hline 230 & 0 & 470 & 0 & 0 & 700 & 0 & 17 & 3,600 & 0 & 0 & 3,600 & 17 \\
\hline 8.5 & 0 & 56 & 0 & 0 & 65 & 0 & 7.3 & 150 & 0 & 0 & 150 & 8.3 \\
\hline 1.0 & 0 & .8 & 0 & 0 & 1.8 & 0 & .7 & 280 & 0 & 0 & 280 & .7 \\
\hline 5,900 & 350 & 27,000 & 4,900 & 71 & 33,000 & 5,200 & 2,900 & 110,000 & 32,000 & 71 & 140,000 & 3,200 \\
\hline 6,000 & 360 & 27,000 & 5,000 & 71 & 33,000 & 5,400 & 3,000 & 110,000 & 33,000 & 71 & $140,000 \mid$ & 3,200 \\
\hline
\end{tabular}

trial water, by regions, 1960

lons per day]

\begin{tabular}{|c|c|c|c|c|c|c|c|c|c|c|c|c|}
\hline \multicolumn{8}{|c|}{ Other uses } & \multirow{2}{*}{\multicolumn{4}{|c|}{$\begin{array}{l}\text { All industrial uses } \\
\text { Water withdrawn }\end{array}$}} & \multirow{4}{*}{$\begin{array}{c}\text { Water } \\
\text { con- } \\
\text { sumed }\end{array}$} \\
\hline \multicolumn{7}{|c|}{ Water withdrawn } & \multirow{3}{*}{$\begin{array}{l}\text { Water } \\
\text { con- } \\
\text { sumed }\end{array}$} & & & & & \\
\hline \multicolumn{2}{|c|}{ Ground water } & \multicolumn{2}{|c|}{ Surface water } & \multirow{2}{*}{$\begin{array}{l}\text { Sew } \\
\text { age }\end{array}$} & \multicolumn{2}{|c|}{ All water } & & \multirow{2}{*}{ Fresh } & \multirow{2}{*}{ Saline } & \multirow{2}{*}{$\begin{array}{l}\text { Sew- } \\
\text { age }\end{array}$} & \multirow{2}{*}{$\begin{array}{c}\text { All } \\
\text { water }\end{array}$} & \\
\hline Fresh & Saline & Fresh & Saline & & Fresh & Saline & & & & & & \\
\hline 130 & 0 & 1,100 & 210 & 0 & 1,200 & 210 & 84 & 1,900 & 3,400 & 0 & 5,300 & 85 \\
\hline 410 & 12 & 1,900 & 2,900 & 0 & 2,300 & 2,900 & 340 & 7,500 & 9,000 & 0 & 16,000 & 350 \\
\hline 220 & 0 & 930 & 780 & 70 & 1,100 & 780 & 120 & 4,100 & 1,900 & 70 & 6,100 & 120 \\
\hline 830 & 0 & 1,200 & 130 & 0 & 2,000 & 130 & 260 & 7,200 & 3,800 & 0 & 11,000 & 260 \\
\hline 310 & 0 & 770 & 260 & 0 & 1,100 & 260 & 170 & 4,300 & 880 & 0 & 5,200 & 180 \\
\hline
\end{tabular}


Table 8. - Self-supplied industrial

\begin{tabular}{|c|c|c|c|c|c|c|c|}
\hline \multirow{4}{*}{ Region } & \multicolumn{7}{|c|}{ Fuel-electric power (public utility) use } \\
\hline & \multicolumn{6}{|c|}{ Water withdrawn } & \multirow{3}{*}{$\begin{array}{l}\text { Water } \\
\text { con- } \\
\text { sumed }\end{array}$} \\
\hline & \multicolumn{2}{|c|}{ Ground water } & \multicolumn{2}{|c|}{ Surface water } & \multicolumn{2}{|c|}{$\begin{array}{l}\text { Ground and Sur- } \\
\text { face water }\end{array}$} & \\
\hline & Fresh & Saline & Fresh & Saline & Fresh & Saline & \\
\hline Tennessee-Cumberland & 0 & 0 & 5,600 & $d$ & 5,600 & 0 & 0 \\
\hline Ohio & 19 & 0 & 15,000 & d & 15,000 & 0 & 33 \\
\hline Eastern Great Lakes-St. Lawrence_ & 0 & 0 & 7,800 & d & 7,800 & 0 & 9 \\
\hline Western Great Lakes & 0 & 0 & 9,700 & 0 & 9,700 & 0 & 3 \\
\hline Hudson Bay & 3 & 0 & & 0 & & 0 & 2 \\
\hline Upper Mississippi & 7 & 0 & 8,200 & 0 & 8,200 & 7 & 4 \\
\hline Upper Missouri & 4 & 0 & 1,100 & $d$ & 1,100 & 0 & 12 \\
\hline Lower Missouri & 1 & 0 & 1,100 & 0 & 1,100 & 0 & 0 \\
\hline Lower Mississippi & 21 & 0 & 930 & 1,600 & 950 & 1,600 & 19 \\
\hline Upper Arkansas-Red & 28 & 0 & 230 & 120 & 260 & 120 & 17 \\
\hline Lower Arkansas-Red-White & 9 & 0 & 2,900 & 3 & 3,000 & 3 & 12 \\
\hline Western Gulf & 480 & 0 & 2,000 & 1,400 & 2,500 & 1,400 & 56 \\
\hline Colorado & 18 & 3 & 120 & d & 130 & 3 & 15 \\
\hline Great Basin & 0 & 0 & 76 & 0 & 76 & 0 & 2 \\
\hline South Pacific & 290 & 0 & 140 & 8,600 & 430 & 8,600 & 17 \\
\hline Pacific Northwest & 0 & 0 & 7 & 0 & 7 & 0 & 0 \\
\hline Hawaii & 14 & 16 & 12 & 260 & 26 & 280 & 0 \\
\hline Alaska & 0 & 0 & 86 & 0 & 86 & 0 & 0 \\
\hline Puerto Rico and Virgin Islands .... & 0 & 0 & 4 & 490 & 4 & 490 & 0 \\
\hline $\begin{array}{l}\text { United States excluding Alaska, } \\
\text { Hawaii, Puerto Rico and Virgin } \\
\text { Islands. }\end{array}$ & 910 & 3 & 72,000 & 26,000 & 73,000 & 26,000 & 220 \\
\hline United States & 920 & 19 & 72,000 & 27,000 & 73,000 & 27,000 & 220 \\
\hline
\end{tabular}


water, by reqions, 1960-Continued

\begin{tabular}{|c|c|c|c|c|c|c|c|c|c|c|c|c|}
\hline \multicolumn{8}{|c|}{ Other uses } & \multicolumn{5}{|c|}{ All industrial uses } \\
\hline \multicolumn{7}{|c|}{ Water withdrawn } & \multirow{3}{*}{$\begin{array}{l}\text { Water } \\
\text { con- } \\
\text { sumed }\end{array}$} & \multicolumn{4}{|c|}{ Water withdrawn } & \multirow{3}{*}{$\begin{array}{c}\text { Water } \\
\text { con- } \\
\text { sumed }\end{array}$} \\
\hline \multicolumn{2}{|c|}{ Ground water } & \multicolumn{2}{|c|}{ Surface water } & \multirow{2}{*}{$\begin{array}{l}\text { Sew- } \\
\text { age }\end{array}$} & \multicolumn{2}{|c|}{ All water } & & \multirow{2}{*}{ Fresh } & \multirow{2}{*}{ Saline } & \multirow{2}{*}{$\begin{array}{l}\text { Sew - } \\
\text { age }\end{array}$} & \multirow{2}{*}{$\begin{array}{c}\text { All } \\
\text { water }\end{array}$} & \\
\hline Fresh & Saline & Fresh & Saline & & Fresh & Saline & & & & & & \\
\hline 230 & 0 & 1,200 & 0 & 0 & 1,500 & 0 & 240 & 7,100 & 0 & 0 & 7,100 & 240 \\
\hline 600 & 34 & 6,600 & .3 & 0 & 7,200 & 34 & 310 & 22,000 & 34 & 0 & 22,000 & 340 \\
\hline 81 & 13 & 3,000 & 0 & 0 & 3,100 & 13 & 100 & 11,000 & 13 & 0 & 11,000 & 110 \\
\hline 340 & 12 & 4,200 & 0 & 0 & 4,600 & 12 & 180 & 14,000 & 12 & 0 & 14,000 & 190 \\
\hline 4.9 & 6.0 & 80 & 0 & 0 & 85 & 6.0 & 6.8 & 88 & 6.0 & 0 & & 8.8 \\
\hline 480 & 20 & 1,200 & 0 & 0 & 1,700 & 20 & 36 & 9,900 & 20 & 0 & 9,900 & 40 \\
\hline 140 & 13 & 220 & 5.0 & 0 & 360 & 18 & 49 & 1,500 & 18 & 0 & 1,500 & 61 \\
\hline 43 & 0 & 60 & 0 & 0 & 100 & 0 & 6.1 & 1,200 & 0 & 0 & 1,200 & 6.1 \\
\hline 450 & 25 & 940 & 0 & 0 & 1,400 & 25 & 380 & 2,300 & 1,600 & 0 & 4,000 & 390 \\
\hline 110 & 20 & 91 & 15 & 0 & 200 & 36 & 35 & 460 & 150 & 0 & 610 & 52 \\
\hline 200 & 38 & 590 & 1.0 & 0 & 790 & 38 & 150 & 3,700 & 42 & 0 & 3,800 & 160 \\
\hline 430 & 14 & 830 & 3.4 & 0 & 1,300 & 17 & 270 & 3,700 & 1,500 & 0 & 5,200 & 330 \\
\hline 80 & 3.3 & 53 & 0 & .2 & 130 & 3.3 & 37 & 270 & 6.3 & .2 & 270 & 52 \\
\hline 110 & 6.0 & 200 & 5.5 & 0 & 310 & 12 & 9.1 & 390 & 12 & 0 & 400 & 11 \\
\hline 300 & 140 & 64 & 510 & .5 & 360 & 640 & 80 & 790 & 9,300 & .5 & 10,000 & 97 \\
\hline 400 & 0 & 1,700 & 48 & 0 & 2,100 & 48 & 91 & 2,100 & 48 & 0 & 2,100 & 91 \\
\hline 110 & 5.0 & 33 & .1 & 0 & 140 & 5.1 & 13 & 170 & 280 & 0 & 450 & 13 \\
\hline 12 & 0 & 70 & 0 & & 82 & 0 & 0 & 170 & 0 & 0 & 170 & 0 \\
\hline 29 & 1.6 & 130 & 160 & 0 & 160 & 160 & 6.9 & 160 & 650 & 0 & 810 & 6.9 \\
\hline 5,900 & 350 & 27,000 & 4,900 & 71 & 33,000 & 5,200 & 2,900 & 110,000 & 32,000 & 71 & 140,000 & 3,200 \\
\hline 6,000 & 360 & 27,000 & 5,000 & 71 & 33,000 & 5,400 & 3,000 & 110,000 & 33,000 & 71 & 140,000 & 3,200 \\
\hline
\end{tabular}


Table 9. -Water used for public utility generation of fuel electric power, by States, 1960

[Million gallons per day]

\begin{tabular}{|c|c|c|c|c|c|c|c|c|c|c|c|c|}
\hline & \multicolumn{6}{|c|}{ Condenser cooling } & \multicolumn{5}{|c|}{ Otheì uses } & \multirow{4}{*}{$\begin{array}{c}\text { Con- } \\
\text { sumed } \\
\end{array}$} \\
\hline & \multicolumn{4}{|c|}{ Self-supplied } & \multirow{3}{*}{$\begin{array}{c}\text { Public } \\
\text { supplies }\end{array}$} & \multirow{3}{*}{$\begin{array}{c}\text { Self- } \\
\text { supplied } \\
\text { and } \\
\text { public } \\
\text { supplies }\end{array}$} & \multicolumn{3}{|c|}{ Self-supplied } & \multirow{3}{*}{$\begin{array}{c}\text { Public } \\
\text { supplies }\end{array}$} & \multirow{3}{*}{$\begin{array}{c}\text { Self- } \\
\text { supplied } \\
\text { and } \\
\text { public } \\
\text { supplies }\end{array}$} & \\
\hline & Grounc & water & Surfac & water & & & \multirow{2}{*}{$\begin{array}{c}\text { Ground } \\
\text { water } \\
\text { fresh }\end{array}$} & \multicolumn{2}{|c|}{ Surface water } & & & \\
\hline & Fresh & Saline & Fresh & Saline & & & & Fresh & Saline & & & \\
\hline Alabama__... & 0 & 0 & 2,900 & 140 & 0 & 3,100 & 1 & 91 & 0 & 0 & 92 & 0 \\
\hline Alaska $\ldots \ldots \ldots$ & 0 & 0 & 86 & 0 & 0 & 86 & 0 & 0 & 0 & 0 & 0 & 0 \\
\hline Arizona _... & 18 & 0 & 33 & 0 & 0 & 51 & 0 & 0 & 0 & 0 & 0 & 12 \\
\hline Arkansas _. & 7 & 0 & 270 & 0 & 0 & 280 & 0 & 0 & 0 & 0 & 0 & 7 \\
\hline California $\ldots \ldots \ldots$ & 290 & 0 & 140 & 8,600 & 100 & 9,200 & 0 & 1 & 0 & 13 & 14 & 17 \\
\hline Colorado _........ & 2 & 0 & 160 & 0 & 40 & 200 & 0 & 0 & 0 & 0 & 0 & 7 \\
\hline Connecticut _....... & 0 & 0 & 580 & 940 & 0 & 1,500 & 0 & 0 & 0 & 1 & 1 & 0 \\
\hline Delaware $\ldots \ldots \ldots$ & 0 & 0 & 0 & 440 & 0 & 440 & 2 & 0 & 0 & 0 & 2 & 0 \\
\hline Florida__. & 6 & 0 & 1,700 & 3,100 & 690 & 5,500 & 2 & 1 & 0 & 2 & 5 & 2 \\
\hline Georgia & 3 & 0 & 1,400 & 320 & 0 & 1,800 & 0 & 0 & 0 & 0 & 0 & 0 \\
\hline Hawaii & 14 & 16 & 12 & 260 & 0 & 300 & 0 & 0 & 0 & 2 & 2 & 0 \\
\hline Idaho. & 0 & 0 & 0 & 0 & 0 & 0 & 0 & 0 & 0 & 0 & 0 & 0 \\
\hline Illinois & 0 & 0 & 9,400 & 0 & 1 & 9,400 & 8 & 320 & 0 & 2 & 320 & 2 \\
\hline Indiana & 0 & 0 & 3,200 & 0 & 0 & 3,200 & 0 & 41 & 0 & 0 & 41 & 7 \\
\hline Iowa & 0 & 0 & 1,500 & 0 & 2 & 1,500 & 0 & 2 & 0 & 2 & $\therefore 4$ & 2 \\
\hline Kansas $\ldots \ldots \ldots$ & 23 & 0 & 510 & 0 & 0 & 530 & 1 & 3 & 0 & 0 & 4 & 12 \\
\hline Kentucky _...... & 0 & 0 & 2,000 & 0 & 0 & 2,000 & 0 & 1 & 0 & 0 & $i$ & 1 \\
\hline Louisiana_........... & 22 & 0 & 3,000 & 1,700 & 5 & 4,700 & 6 & 0 & 0 & 0 & 6 & 11 \\
\hline Maine $\ldots \ldots \ldots$ & 0 & 0 & 0 & 120 & 0 & 120 & 0 & 1 & 0 & 1 & 2 & 0 \\
\hline Maryland ........ & 0 & 0 & 500 & 560 & 0 & 1,100 & 0 & 0 & 22 & 1 & 23 & 0 \\
\hline Massachusetts & 0 & 0 & 160 & 1,600 & 9 & 1,800 & 0 & 0 & 3 & 6 & 9 & 1 \\
\hline Michigan _....... & 0 & 0 & 3,900 & 0 & 0 & 3,900 & 0 & 24 & 0 & 1 & 25 & 1 \\
\hline Minnesota $\ldots \ldots \ldots$ & 0 & 0 & 1,200 & 0 & 0 & 1,200 & 0 & 22 & 0 & 0 & 22 & 0 \\
\hline Mississippi & 6 & 0 & 110 & 160 & 0 & 270 & 0 & 0 & 0 & 0 & 0 & 10 \\
\hline Missouri & 1 & 0 & 1,300 & 0 & 2 & 1,300 & 0 & 4 & 0 & 2 & 6 & 1 \\
\hline
\end{tabular}




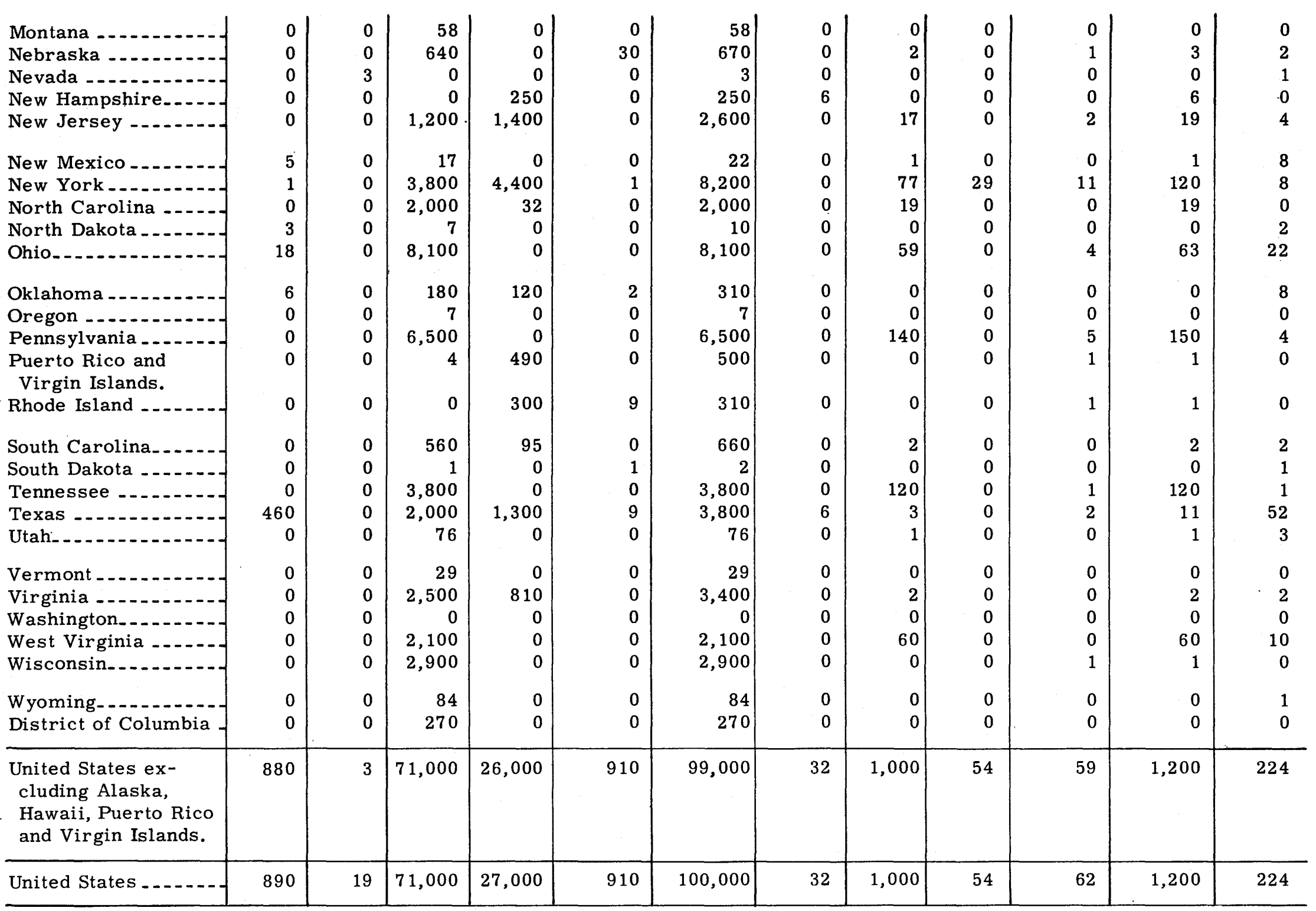


Table 10.-Water used for public utility generation of fuel electric power, by regions, 1960

[Million gallons per day]

\begin{tabular}{|c|c|c|c|c|c|c|c|c|c|c|c|c|}
\hline \multirow{4}{*}{ Region } & \multicolumn{6}{|c|}{ Condenser cooling } & \multicolumn{5}{|c|}{ Other uses } & \multirow{4}{*}{$\begin{array}{l}\text { Con- } \\
\text { sumed }\end{array}$} \\
\hline & \multicolumn{4}{|c|}{ Self-supplied } & \multirow{3}{*}{$\begin{array}{l}\text { Public } \\
\text { supplies }\end{array}$} & \multirow{3}{*}{$\begin{array}{l}\text { Self- } \\
\text { supplied } \\
\text { and } \\
\text { public } \\
\text { supplies }\end{array}$} & \multicolumn{3}{|c|}{ Self-supplied } & \multirow{3}{*}{$\begin{array}{l}\text { Public } \\
\text { supplies }\end{array}$} & \multirow{3}{*}{$\begin{array}{l}\text { Self- } \\
\text { supplied } \\
\text { and } \\
\text { public } \\
\text { supplies }\end{array}$} & \\
\hline & \multicolumn{2}{|c|}{ Ground water } & \multicolumn{2}{|c|}{ Surface water } & & & \multirow{2}{*}{$\begin{array}{l}\text { Ground } \\
\text { water } \\
\text { fresh }\end{array}$} & \multicolumn{2}{|c|}{ Surface water } & & & \\
\hline & Fresh & Saline & Fresh & Saline & & & & Fresh & Saline & & & \\
\hline New England & 0 & 0 & 620 & 3,200 & 18 & 3,900 & 6 & 1 & 3 & 9 & 19 & 1 \\
\hline 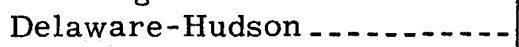 & 1 & 0 & 5,000 & 6,000 & 1 & 11,000 & 2 & 150 & 29 & 13 & 190 & 13 \\
\hline Chesapeake & 0 & 0 & 2,900 & 1,100 & 0 & 4,000 & 0 & 40 & 22 & 3 & 65 & 2 \\
\hline South Atlantic & 9 & 0 & 5,200 & 3,600 & 690 & 9,500 & 1 & 23 & 0 & 2 & 26 & 3 \\
\hline Eastern Gulf & 5 & 0 & 3,200 & 610 & 0 & 3,900 & 2 & 1 & 0 & 0 & 3 & 4 \\
\hline Tennessee-Cumberland...... & 0 & 0 & 5,400 & 0 & 0 & 5,400 & 0 & 210 & 0 & 0 & 210 & 0 \\
\hline Ohio & 18 & 0 & 15,000 & 0 & 0 & 15,000 & 1 & 120 & 0 & 1 & 120 & 33 \\
\hline $\begin{array}{l}\text { Eastern Great Lakes-St. } \\
\text { Lawrence. }\end{array}$ & 0 & 0 & 7,800 & 0 & 0 & 7,800 & 0 & 75 & 0 & 6 & 81 & 9 \\
\hline Western Great Lakes........ & 0 & 0 & 9,500 & 0 & 0 & 9,500 & 0 & 170 & 0 & 3 & 170 & 3 \\
\hline Hudson Bay & 3 & 0 & & 0 & 0 & & 0 & 0 & 0 & 0 & 0 & 2 \\
\hline Upper Mississippi & 0 & 0 & 8,000 & 0 & 3 & 8,000 & 7 & 210 & 0 & 4 & 220 & 4 \\
\hline Upper Missouri_. & 4 & 0 & 1,100 & 0 & 70 & 1,200 & 0 & 5 & 0 & 1 & 6 & 12 \\
\hline Lower Missouri _.......... & 1 & 0 & 1,100 & 0 & 2 & 1,100 & 0 & 0 & 0 & 1 & 1 & 0 \\
\hline Lower Mississippi & 15 & 0 & 930 & 1,600 & 0 & 2,600 & 6 & 0 & 0 & 1 & 7 & 19 \\
\hline Upper Arkansas-Red ........ & 27 & 0 & 230 & 120 & 2 & 380 & 1 & 0 & 0 & 0 & 1 & 17 \\
\hline Lower Arkansas-Red-White _- & 9 & 0 & 2,900 & 3 & 7 & 3,000 & 0 & 0 & 0 & 0 & 0 & 12 \\
\hline Western Gulf $\ldots$ & 470 & 0 & 2,000 & 1,400 & 8 & 3,900 & 6 & 3 & 0 & 2 & 11 & 56 \\
\hline Colorado_... & 18 & 3 & 120 & 0 & 0 & 140 & 0 & 1 & 0 & 0 & 1 & 15 \\
\hline Great Basin & 0 & 0 & 75 & 0 & 0 & 75 & 0 & 1 & 0 & 0 & 1 & 2 \\
\hline South Pacific & 290 & 0 & 140 & 8,600 & 100 & 9,200 & 0 & 1 & 0 & 13 & 14 & 17 \\
\hline Pacific Northwest__. & 0 & 0 & 7 & 0 & 0 & 7 & 0 & 0 & 0 & 0 & 0 & 0 \\
\hline Hawaii. & 14 & 16 & 12 & 260 & 0 & 300 & 0 & 0 & 0 & 2 & 2 & 0 \\
\hline Alaska. & 0 & 0 & 86 & 0 & 0 & 86 & 0 & 0 & 0 & 0 & 0 & 0 \\
\hline Puerto Rico and Virgin Islands & 0 & 0 & 4 & 490 & 0 & 500 & 0 & 0 & 0 & 1 & 1 & 0 \\
\hline $\begin{array}{l}\text { United States excluding } \\
\text { Alaska, Hawaii, Puerto Rico } \\
\text { and Virgin Islands. }\end{array}$ & 880 & 3 & 71,000 & 26,000 & 910 & 99,000 & 32 & 1,000 & 54 & 59 & 1,200 & 224 \\
\hline United States $\ldots \ldots \ldots$ & 890 & 19 & 71,000 & 27,000 & 910 & 100,000 & 32 & 1,000 & 54 & 62 & 1,200 & 224 \\
\hline
\end{tabular}




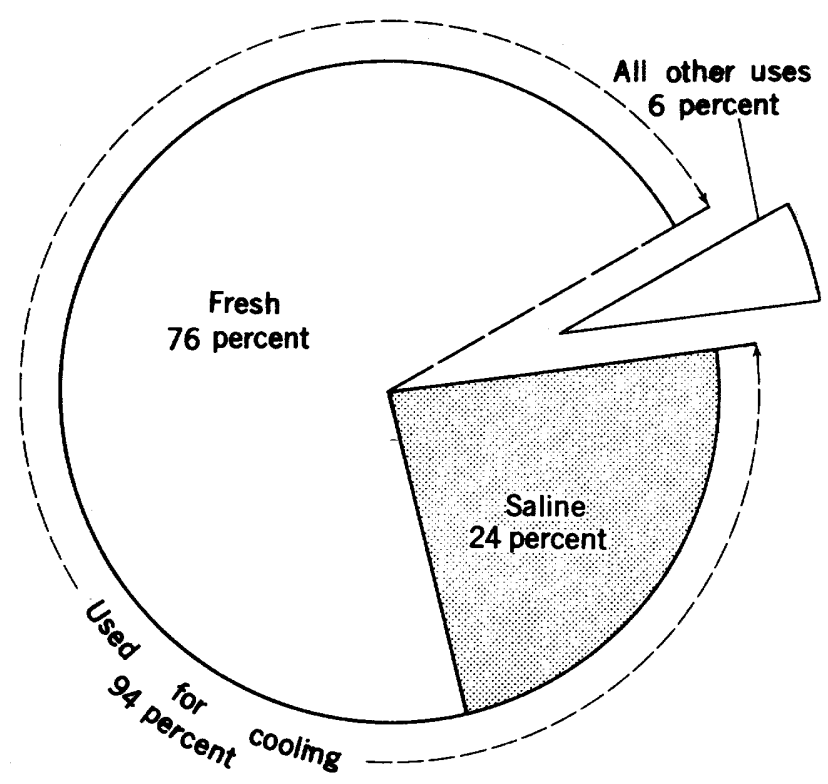

Figure 4. - Most industrial water is used for cooling.

Almost all the water used for cooling condensers was obtained from surface sources, and about one-fourth was saline. Less than 1 percent of the water withdrawn was consumed. The effect of differences in climate and availability of water in the West and in the East is reflected in the kind of water used and how it is used for generation of electric power. Although consumption is very small, the percent of intake consumed is several times greater in the dry West than in the humid East. About 5 percent of the water withdrawn in the West is from wells, whereas only about one-tenth of 1 percent of the water withdrawn in the East is from wells. About two-thirds of the water withdrawn in the West is saline, whereas in the East, where the supply of water is greater, only about onefifth of the water withdrawn is saline.

\section{AIR CONDITIONING}

About 560 of the $1,500 \mathrm{mgd}$ of water used for air conditioning was taken from public supplies; the remainder was self-supplied (tables 11 and 12). The $560 \mathrm{mgd}$ from public supplies is part of the $6,600 \mathrm{mgd}$ used by industry (tables 1 and 2). The $920 \mathrm{mgd}$ of selfsupplied water for air conditioning is included in the $140,000 \mathrm{mgd}$ self-supplied water which industry uses for all purposes (tables 7 and 8).
The quantities given in tables 11 and 12 are annual rates. Air conditioning is seasonal, most of the water being used in a 4- to 6month period. Therefore, during the airconditioning season, water is used at a much higher rate than that shown in tables 11 and 12; during the rest of the year, little or no water is used. The effect of seasonal demand on self-supplied water supplies is usually not serious; however, it may have a serious effect on public supplies. For example, the capacity to supply the air-conditioning demand becomes a problem. Seidel and Carpenter (1958) state that the air-conditioning season in Ames, Iowa, comprises possibly 10 percent of the year and facilities provided to serve these unusual demands would be idle and unproductive the remaining 90 percent of the time. Because the facilities are idle such a great part of the time, air-conditioning demands do not carry their full share of the cost unless some kind of demand charge is applied. A study in Grand Rapids, Mich., showed that air-conditioning requirements were 22 percent of the demands on maximum days but required only 4 percent of the annual demands (Am. Water Works Assoc. Committee on Water Use in Air Conditioning and Other Refrigeration, 1958).

\section{WATERPOWER}

About 2,200 million acre-feet of water was used during 1960 to generate waterpower (tables 13 and 14). The rate of use is variable and depends on the water available and the demand for power. The quantities of water used were computed with the aid of two publications of the U.S. Federal Power Commission (1957a and 1960-61). The average plant efficiency was estimated. Much of the water was used more than once.

Waterpower is considered a nonconsumptive use; however, many reservoirs are built to store water for power. The amount of evaporation from these reservoirs is very large, estimated at 9,000 mgd during 1954 . This is almost half the quantity of water withdrawn for all public supplies in the United States in 1960 and 2.5 times the quantity consumed. 
Table 11.-Water withdrawn for air conditionina, by States, 1960

[Million gallons per day]

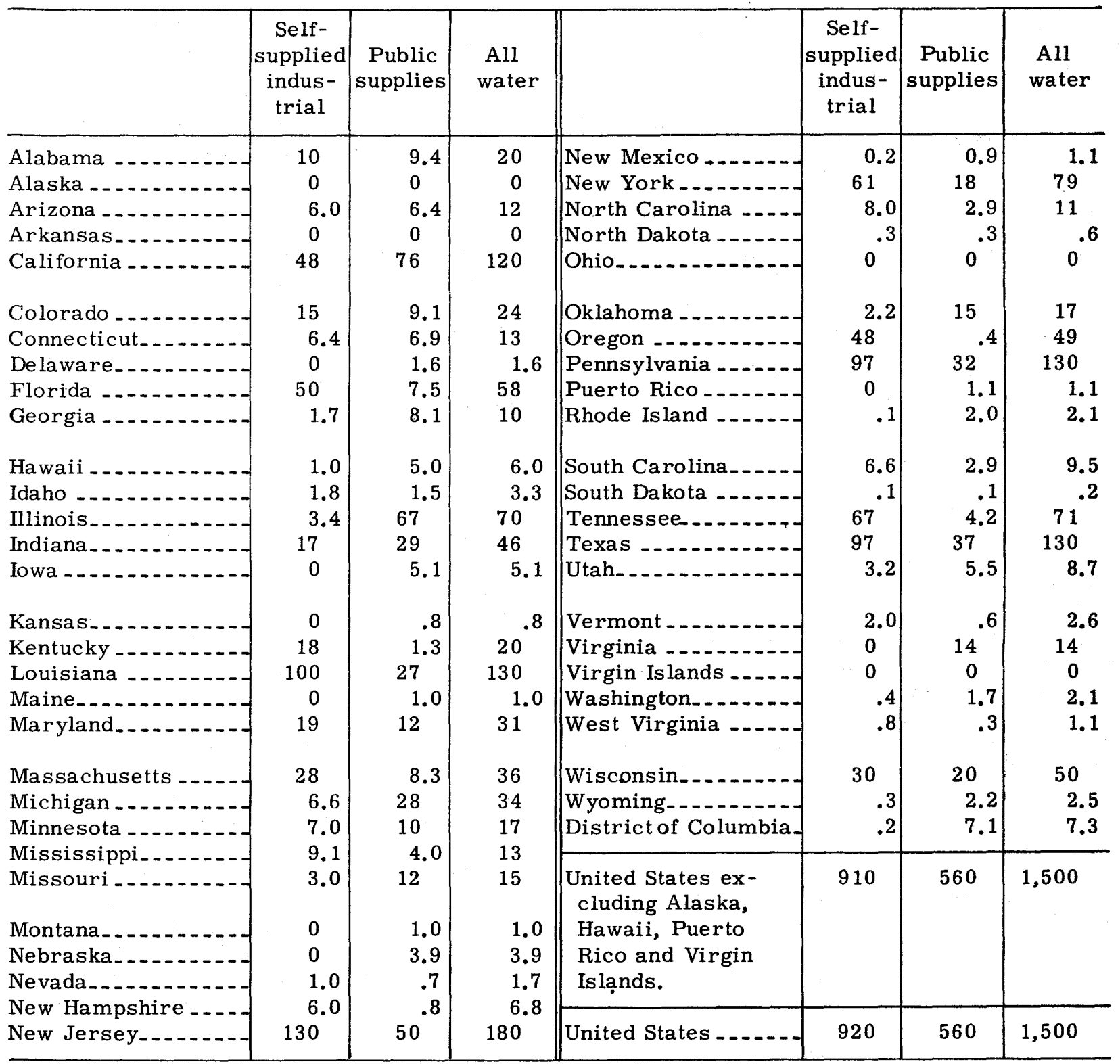


Table 12.-Water withdrawn for air conditioning, by reaions, 1960

[Million gallons per day]

\begin{tabular}{|c|c|c|c|c|c|c|c|}
\hline Region & $\begin{array}{c}\text { Self- } \\
\text { supplied } \\
\text { indus- } \\
\text { trial }\end{array}$ & $\begin{array}{c}\text { Public } \\
\text { supplies }\end{array}$ & $\begin{array}{c}\text { All } \\
\text { water }\end{array}$ & Region & $\begin{array}{c}\text { Self- } \\
\text { supplied } \\
\text { indus- } \\
\text { trial }\end{array}$ & $\begin{array}{c}\text { Public } \\
\text { supplies }\end{array}$ & $\begin{array}{l}\text { All } \\
\text { water }\end{array}$ \\
\hline New England...... & 41 & 19 & 60 & Lower Arkansas - & 31 & 13 & 44 \\
\hline Delaware-Hudson - & 200 & 84 & 280 & Red-White. & & & \\
\hline Chesapeake & 34 & 32 & 66 & Western Gulf _..... & 110 & 36 & 150 \\
\hline South Atlantic.... & 41 & 24 & 65 & Colorado........ & 7.9 & 8.5 & 16 \\
\hline Eastern Gulf & 36 & 17 & 52 & Great Basin & 3.5 & 5.8 & 9.3 \\
\hline Tennessee- & & 4.5 & 63 & South Pacific & 47 & 76 & 120 \\
\hline Cumberland. & & & & Pacific Northwest. & 51 & 3.2 & 54 \\
\hline Ohio & 88 & 27 & 120 & Hawaii_... & 1.0 & 5.0 & 6.0 \\
\hline Eastern Great Lakes - & 18 & 24 & 42 & Alaska_... & 0 & 0 & 0 \\
\hline $\begin{array}{l}\text { St. Lawrence. } \\
\text { Western Great Lakes }\end{array}$ & 45 & 92 & 140 & $\begin{array}{l}\text { Puerto Rico and } \\
\text { Virgin Islands. }\end{array}$ & 0 & 1.1 & 1.1 \\
\hline Hudson Bay....... & 1.2 & .8 & 2.0 & & 010 & 560 & 1500 \\
\hline Upper Mississippi_-_- & 7.0 & 34 & 42 & cluding Alaska, & 80 & 800 & 1,000 \\
\hline Upper Missouri & 13 & 14 & 27 & Hawaii, Puerto Rico & & & \\
\hline Lower Missouri.... & 2.0 & 3.3 & 5.3 & and Virgin Islands. & & & \\
\hline Lower Mississippi -- & 68 & 23 & 91 & & & & \\
\hline Upper Arkansas-Red & 13 & 14 & 26 & United States & 920 & 560 & 1,500 \\
\hline
\end{tabular}

SUMMARY OF WITHDRAWAL USES

The estimated withdrawal of water in the United States amounted to 270,000 mgd during 1960 , exclusive of water used for waterpower (tables 15 and 16). This amounts to $1,500 \mathrm{gpd}$ per person. Surface-water sources supplied $220,000 \mathrm{mgd}$, and ground water supplied $47,000 \mathrm{mgd}$. Industry used the largest part of the water withdrawn-140,000 of the $270,000 \mathrm{mgd}$ (fig. 5). Irrigation used the next greatest amount-110,000 mgd including conveyance losses. Rural, domestic, and stock use was the smallest- $3,600 \mathrm{mgd}$.

The withdrawal of water was the greatest in the eastern industrial areas and in western areas where irrigation is practiced extensively. Withdrawals in the midcontinent region were the smallest. Only about onefourth of the water withdrawn was consumed; 61,000 of the $270,000 \mathrm{mgd}$ withdrawn.

Most of the water consumed was used for irrigation, a fact of added importance when we consider that most irrigation is practiced at times and places where the water supply is likely to be inadequate. Generally a greater percentage of the water withdrawn for any use is consumed in areas having a dry climate than in humid areas (fig. 6), and a greater percentage is consumed in the hot, dry part of the year. Irrigation consumed the largest quantity of water, $52,000 \mathrm{mgd}$; public supplies the next largest amount, $3,500 \mathrm{mgd}$; and rural uses consumed the least, $2,800 \mathrm{mgd}$. 

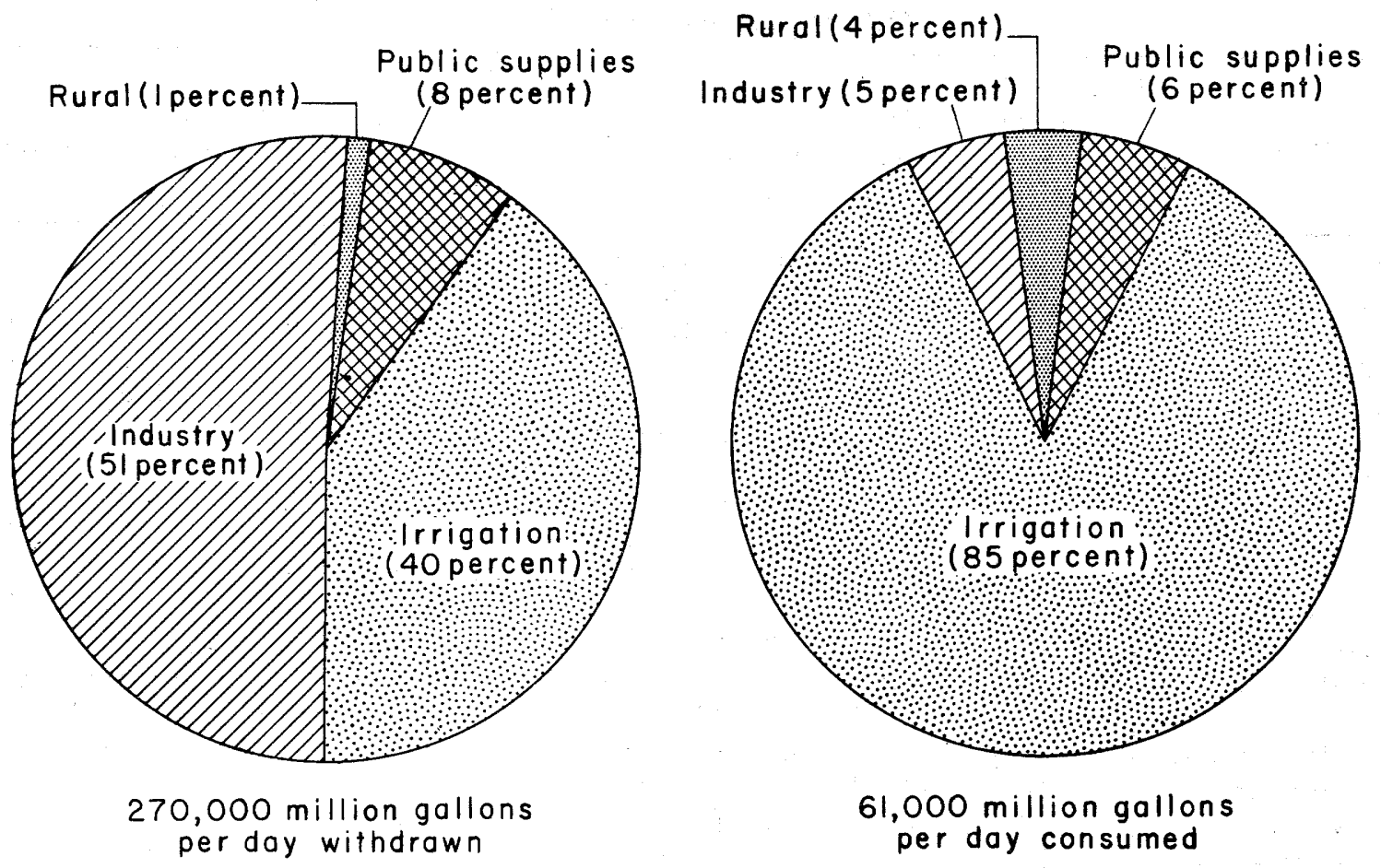

Figure 5. - How water was used in 1960.

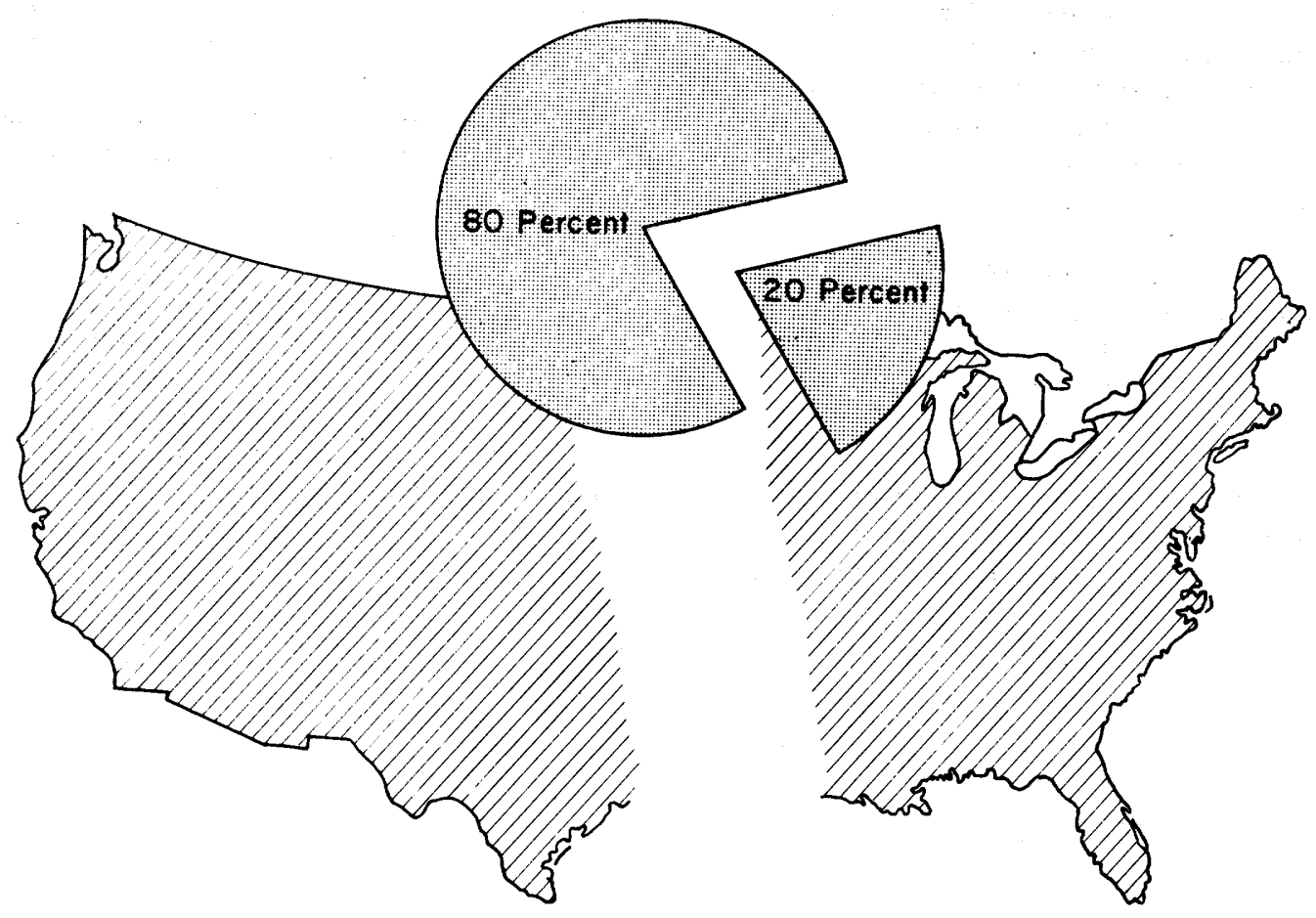

Figure 6. -Where water is consumed. 
Table 13. - Water used for waterpower, by States, 1960

\begin{tabular}{|c|c|c|c|c|c|}
\hline & Mgd & $\mid \begin{array}{c}1,000 \text { 's of } \\
\text { acre- } \mathrm{ft} \\
\text { per year }\end{array}$ & & Mgd & $\begin{array}{c}1,000 \text { 's of } \\
\text { acre-ft } \\
\text { per year }\end{array}$ \\
\hline Alabama ..... & 120,000 & 130,000 & New Jersey & 1,200 & 1,300 \\
\hline Alaska ....... & 370 & 410 & New Mexico & 520 & 580 \\
\hline Arizona...... & 14,000 & 16,000 & New York & 270,000 & 310,000 \\
\hline Arkansas..... & 8,200 & 9,200 & North Carolina & 50,000 & 56,000 \\
\hline California .... & 67,000 & 75,000 & North Dakota & 7,700 & 8,600 \\
\hline Colorado & 3,200 & 3,500 & Ohio & 780 & 870 \\
\hline Connecticut_.- & 8,800 & 9,800 & Oklahoma & 9,300 & 10,000 \\
\hline Delaware..... & 0 & 0 & Oregon & 180,000 & 200,000 \\
\hline Florida & 13,000 & 15,000 & Pennsylvania & 47,000 & 52,000 \\
\hline Georgia ...... & 41,000 & 46,000 & Puerto Rico & 780 & 870 \\
\hline Hawaii ....... & $--n-n$ & ------ & Rhode Island & 430 & 480 \\
\hline Idaho _....... & 120,000 & 130,000 & South Carolina........ & 62,000 & 70,000 \\
\hline Illinois. & 14,000 & 15,000 & South Dakota. & 11,000 & 12,000 \\
\hline Indiana_. & 5,300 & 5,900 & Tennessee & 150,000 & 170,000 \\
\hline Iowa $\ldots .$. & 34,000 & 38,000 & Texas & 17,000 & 19,000 \\
\hline Kansas.... & 1,500 & 1,600 & Utah. & 1,800 & 2,000 \\
\hline Kentucky _.-. & 55,000 & 61,000 & Vermont & 18,000 & 20,000 \\
\hline Louisiana .... & 0 & 0 & Virgin Islands &.------ & - \\
\hline Maine.... & 87,000 & 97,000 & Virginia $\ldots . .$. & 31,000 & 35,000 \\
\hline Maryland....... & 19,000 & 21,000 & Washington. & 170,000 & 190,000 \\
\hline Massachusetts ... & 25,000 & 28,000 & West Virginia $\ldots \ldots$ & 21,000 & 23,000 \\
\hline Michigan ..... & 79,000 & 88,000 & Wisconsin & 99,000 & 110,000 \\
\hline Minnesota & 25,000 & 28,000 & Wyoming & 4,500 & 5,100 \\
\hline Mississippi_. & 0 & 0 & District of Columbia & 27 & 30 \\
\hline Missouri _... & 13,000 & 14,000 & United States excluding & $2,000,000$ & $2,200,000$ \\
\hline Montana_-- & 46,000 & 51,000 & Alaska, Hawaii, Puerto & & \\
\hline Nebraska_-_- & 19,000 & 21,000 & Rico and Virgin Islands. & & \\
\hline $\begin{array}{l}\text { Nevada } \\
\text { New Hampshir }\end{array}$ & $\begin{array}{r}5,500 \\
30,700\end{array}$ & $\begin{array}{r}6,200 \\
34,000\end{array}$ & United States _. & $2,000,000$ & $2,200,000$ \\
\hline New Hamp & 30,900 & 34,000 & & & \\
\hline
\end{tabular}

Table 14. -Water used for waterpower, by regions, 1960

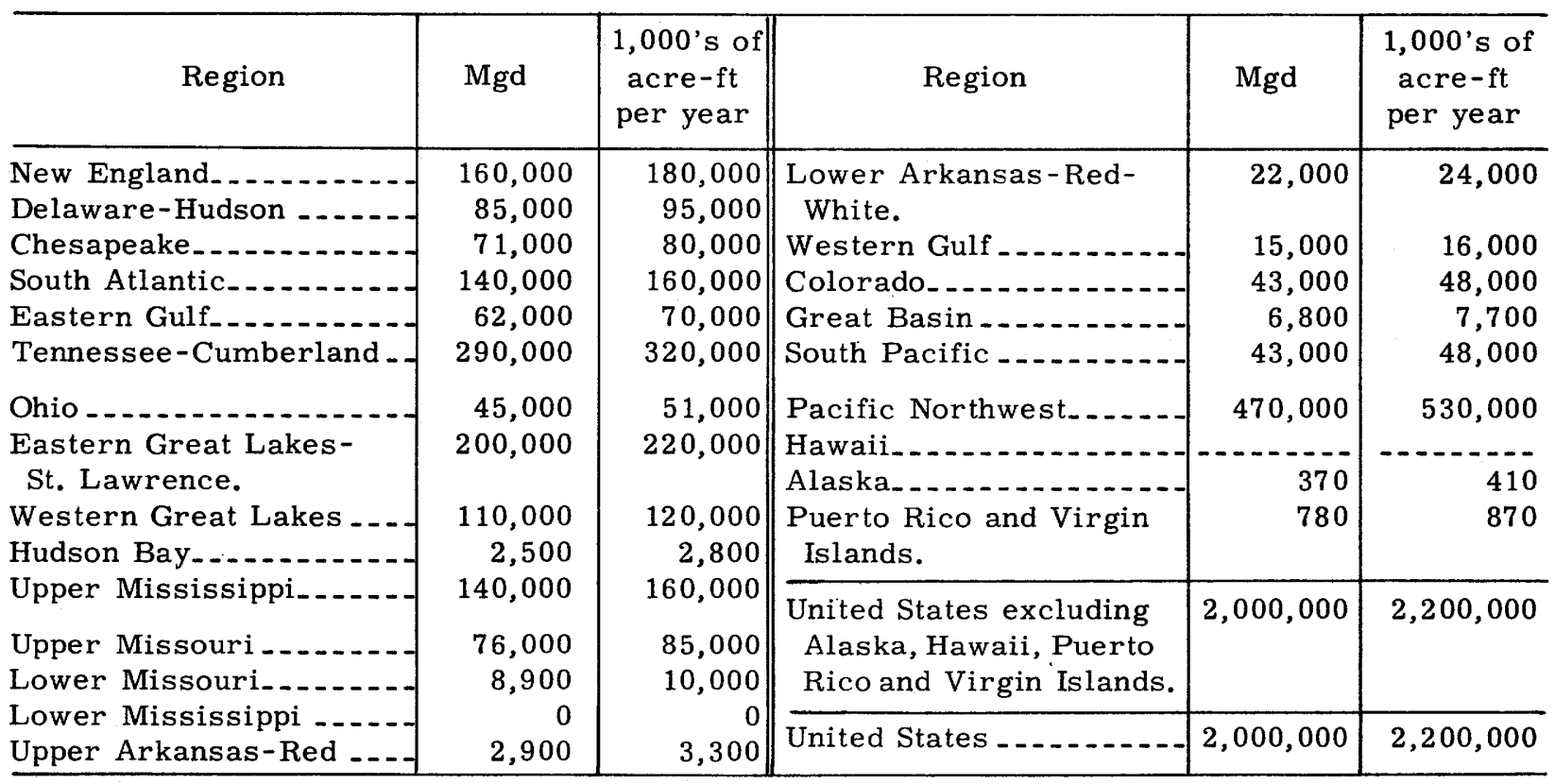


Table 15. - Summary of water withdrawn except for waterpower, by States, 1960

[Million gallons per day]

\begin{tabular}{|c|c|c|c|c|c|c|c|c|c|c|c|c|c|c|}
\hline & \multirow{4}{*}{$\begin{array}{l}\text { Popu- } \\
\text { lation } \\
1,000 \text { 's }\end{array}$} & \multirow{4}{*}{$\begin{array}{c}\text { Per } \\
\text { capita } \\
\text { use } \\
\text { (gpd) }\end{array}$} & \multicolumn{11}{|c|}{ Water withdrawn } & \multirow{4}{*}{$\begin{array}{l}\text { Water } \\
\text { con- } \\
\text { sumed }\end{array}$} \\
\hline & & & \multicolumn{10}{|c|}{ Excluding irrigation conveyance losses } & \multirow{3}{*}{$\begin{array}{l}\text { Including } \\
\text { convey- } \\
\text { ance } \\
\text { losses }\end{array}$} & \\
\hline & & & \multicolumn{3}{|c|}{ Ground water } & \multicolumn{3}{|c|}{ Surface water } & \multirow[b]{2}{*}{$\begin{array}{c}\text { Sew- } \\
\text { age }\end{array}$} & \multicolumn{3}{|c|}{ All sources } & & \\
\hline & & & Fresh & Saline & $\begin{array}{c}\text { Fresh } \\
\text { and } \\
\text { saline }\end{array}$ & Fresh & Saline & $\begin{array}{l}\text { Fresh } \\
\text { and } \\
\text { saline }\end{array}$ & & Fresh & Saline & $\begin{array}{l}\text { Fresh } \\
\text { and } \\
\text { saline }\end{array}$ & & \\
\hline Alaska ... & 226 & 870 & 25 & 0 & 25 & 170 & 0 & 170 & 0 & 200 & 0 & 200 & 200 & .7 \\
\hline Arizona - - & 1,302 & 4,700 & 3,200 & 0 & 3,200 & 1,800 & 0 & 1,800 & 0 & 5,000 & 0 & 5,000 & 6,100 & 3,000 \\
\hline Arkansas... & 1,786 & 900 & 1,000 & 0 & 1,000 & 530 & 0 & 530 & 0 & 1,500 & 0 & 1,500 & 1,600 & 750 \\
\hline California & 15,717 & 2,200 & 11,000 & 140 & 11,000 & 11,000 & 9,100 & 20,000 & .5 & 22,000 & 9,300 & 31,000 & 35,000 & 15,000 \\
\hline Colorado _. & 1,754 & 6,000 & 1,900 & 10 & 1,900 & 7,600 & 10 & 7,700 & 0 & 9,600 & 20 & 9,600 & 11,000 & 5,000 \\
\hline Connecticut.- & 2,535 & 840 & 78 & 0 & 78 & 1,000 & 1,000 & 2,000 & 0 & 1,100 & 1,000 & 2,100 & 2,100 & 120 \\
\hline Delaware... & 446 & 2,100 & 50 & 0 & 50 & 58 & 820 & 880 & 0 & 110 & 820 & 930 & 930 & 62 \\
\hline Florida & 4,952 & 1,400 & 1,600 & 0 & 1,600 & 2,200 & 3,300 & 5,600 & 0 & 3,800 & 3,300 & 7,100 & 7,100 & 1,200 \\
\hline Georgia $\ldots$ & 3,943 & 680 & 430 & 0 & 430 & 1,800 & 430 & 2,300 & 0 & 2,300 & 430 & 2,700 & 2,700 & 170 \\
\hline Hawaii ... & 634 & 2,500 & 580 & 21 & 600 & 600 & 260 & 860 & 0 & 1,200 & 280 & 1,500 & 1,600 & 410 \\
\hline Idaho & 667 & 24,000 & 2,500 & 0 & 2,500 & 8,700 & 0 & 8,700 & 0 & 11,000 & 0 & 11,000 & 16,000 & 4,600 \\
\hline Illinois. . . . & 10,081 & 1,300 & 600 & 40 & 640 & 13,000 & 0 & 13,000 & 0 & 14,000 & 40 & 14,000 & 14,000 & 340 \\
\hline Indiana. & 4,662 & 1,200 & 400 & 11 & 410 & 5,400 & 0 & 5,400 & 0 & 5,800 & 11 & 5,800 & 5,800 & 290 \\
\hline Iowa $\ldots \ldots$ & 2,758 & 740 & 330 & 0 & 330 & 1,700 & 0 & 1,700 & 0 & 2,100 & 0 & 2,100 & 2,100 & 230 \\
\hline Kansas... & 2,179 & 1,400 & 1,200 & 0 & 1,200 & 1,600 & 0 & 1,600 & 0 & 2,800 & 0 & 2,800 & 3,100 & 1,800 \\
\hline Kentucky & 3,038 & 840 & 110 & .6 & 110 & 2,400 & .3 & 2,400 & 0 & 2,500 & .9 & 2,500 & 2,500 & 96 \\
\hline Louisiana & 3,257 & 2,500 & 820 & 39 & 860 & 5,400 & 1,700 & 7,100 & 0 & 6,200 & 1,700 & 7,900 & 8,200 & 1,300 \\
\hline Maine & 969 & 590 & 32 & 0 & 32 & 420 & 120 & 540 & 0 & 450 & 120 & 570 & 570 & 49 \\
\hline Maryland.... & 3,101 & 770 & 110 & 0 & 110 & 930 & 1,300 & 2,200 & 70 & 1,100 & 1,300 & 2,400 & 2,400 & 140 \\
\hline Massachusetts & 5,149 & 570 & 190 & 0 & 190 & 1,000 & 1,800 & 2,800 & 0 & 1,200 & 1,800 & 3,000 & 3,000 & 100 \\
\hline Michigan & 7,823 & 870 & 400 & 14 & 420 & 6,400 & 0 & 6,400 & 0 & 6,800 & 14 & 6,800 & 6,800 & 210 \\
\hline Minnesota ..... & 3,414 & 710 & 330 & 0 & 330 & 2,100 & 0 & $2 ; 100$ & 0 & 2,400 & 0 & 2,400 & 2,400 & 170 \\
\hline Mississippi_... & 2,178 & 550 & 620 & 0 & 620 & 400 & 160 & 560 & 0 & 1,000 & 160 & 1,200 & 1,200 & 560 \\
\hline Missouri & 4,320 & 470 & 190 & 3.0 & 200 & 1,800 & 0 & 1,800 & 0 & 2,000 & 3.0 & 2,000 & 2,000 & 240 \\
\hline
\end{tabular}




\begin{tabular}{|c|c|c|c|c|c|c|c|c|c|c|c|c|c|c|}
\hline Montana........ & 675 & $|11,000|$ & 110 & 1.0 & 110 & 5,400 & 0 & 5,400 & 0 & 5,500 & 1.0 & 5,500 & 7,100 & 2,500 \\
\hline Nebraska_._. & 1,411 & 3,100 & 1,500 & 0 & 1,500 & 1,600 & 0 & 1,600 & 0 & 3,200 & 0 & 3,200 & 4,400 & 1,200 \\
\hline Nevada. & 285 & 8,000 & 360 & 3.0 & 360 & 1,500 & 0 & 1,500 & .2 & 1,900 & 3.0 & 1,900 & 2,300 & 1,000 \\
\hline New Hampshire & 607 & 770 & 32 & 0 & 32 & 190 & 250 & 440 & 0 & 220 & 250 & 470 & 470 & 18 \\
\hline New Jersey & 6,067 & 780 & 530 & 7.6 & 540 & 2,100 & 2,100 & 4,200 & 0 & 2,600 & 2,100 & 4,800 & 4,800 & 240 \\
\hline New Mexico & 951 & 2,700 & 1,000 & .1 & 1,000 & 970 & 0 & 970 & 0 & 2,000 & .1 & 2,000 & 2,600 & 1,400 \\
\hline New York & 16,782 & 810 & 620 & 15 & 640 & 7,200 & 5,700 & 13,000 & 0 & 7,900 & 5,800 & 14,000 & 14,000 & 550 \\
\hline North Carolina $=$ & 4,556 & 600 & 170 & 0 & 170 & 2,500 & 32 & 2,500 & 0 & 2,700 & 32 & 2,700 & 2,700 & 190 \\
\hline North Dakota -.- & 632 & 320 & 36 & 6.0 & 42 & 130 & 0 & 130 & 0 & 170 & 6.0 & 170 & 200 & 110 \\
\hline Ohio $\ldots \ldots \ldots$ & 9,706 & 1,200 & 630 & 0 & 630 & 11,000 & 0 & 11,000 & 0 & 12,000 & 0 & 12,000 & 12,000 & 380 \\
\hline Oklahoma & 2,328 & 420 & 290 & 46 & 340 & 480 & 130 & 620 & 0 & 780 & 180 & 960 & 970 & 350 \\
\hline Oregon_.... & 1,769 & 4,500 & 570 & 0 & 570 & 5,800 & 0 & 5,800 & 0 & 6,400 & 0 & 6,400 & 8,000 & 2,900 \\
\hline Pennsylvania -. & 11,319 & 1,100 & 520 & 0 & 520 & 12,000 & 460 & 12,000 & 0 & 12,000 & 460 & 13,000 & 13,000 & 360 \\
\hline Puerto Rico ... & 2,353 & 510 & 210 & 1.6 & 210 & 320 & 650 & 970 & 0 & 520 & 650 & 1,200 & 1,200 & 280 \\
\hline Rhode Island.-- & 859 & 500 & 27 & 0 & 27 & 100 & 300 & 400 & 0 & 130 & 300 & 430 & 430 & 7.7 \\
\hline South Carolina & 2,383 & 460 & 140 & 0 & 140 & 830 & 120 & 960 & 0 & 980 & 120 & 1,100 & 1,100 & 120 \\
\hline South Dakota_.-- & 681 & 540 & 120 & 3.9 & 120 & 160 & 0 & 160 & 0 & 280 & 3.9 & 280 & 370 & 180 \\
\hline Tenriessee..... & 3,567 & 1,600 & 610 & 0 & 610 & 5,000 & 0 & 5,000 & 0 & 5,700 & 0 & 5,700 & 5,700 & 460 \\
\hline Texas_. $\ldots \ldots \ldots$ & 9,580 & 1,800 & 9,100 & 11 & 9,100 & 4,300 & 1,400 & 5,600 & 0 & 13,000 & 1,400 & 15,000 & 17,000 & 7,000 \\
\hline Utah $\ldots \ldots \ldots$ & 891 & 5,100 & 410 & 3.0 & 420 & 3,400 & 5.5 & 3,400 & 0 & 3,800 & 8.5 & 3,800 & 4,500 & 2,300 \\
\hline Vermont $\ldots$ & 390 & 280 & 28 & 0 & 28 & 82 & 0 & 82 & 0 & 110 & 0 & 110 & 110 & 17 \\
\hline Virginia....... & 3,967 & 1,300 & 180 & 0 & 180 & 4,000 & 900 & 4,800 & 0 & 4,100 & 900 & 5,000 & 5,000 & 120 \\
\hline Virgin Islands & 32 & 59 & .8 & 0 & .8 & 1.1 & 0 & 1.1 & 0 & 1.9 & 0 & 1.9 & 1.9 & 1.6 \\
\hline Washington & 2,853 & 2,200 & 790 & 0 & 790 & 4,400 & 48 & 4,500 & 0 & 5,200 & 48 & 5,300 & 6,300 & 1,400 \\
\hline West Virginia_.-. & 1,860 & 2,500 & 130 & .4 & 130 & 4,600 & 0 & 4,600 & 0 & 4,700 & .4 & 4,700 & 4,700 & 150 \\
\hline Wisconsin ... & 3,952 & 1,000 & 530 & 0 & 530 & 3,600 & 0 & 3,600 & 0 & 4,100 & 0 & 4,100 & 4,100 & 150 \\
\hline Wyoming _...- & 330 & 14,000 & 110 & 0 & 110 & 3,300 & 0 & 3,300 & 0 & 3,400 & 0 & 3,400 & 4,600 & 2,000 \\
\hline $\begin{array}{c}\text { District of } \\
\text { Columbia. }\end{array}$ & 764 & 550 & 1.0 & 0 & 1.0 & 420 & 0 & 420 & 0 & 420 & 0 & 420 & 420 & 16 \\
\hline $\begin{array}{l}\text { United States ex- } \\
\text { cluding Alaska, } \\
\text { Hawaii, Puerto } \\
\text { Rico and Virgin } \\
\text { Islands. }\end{array}$ & 178,463 & 1,500 & 46,000 & 360 & 46,000 & 170,000 & 31,000 & 200,000 & 71 & 210,000 & 32,000 & 240,000 & 270,000 & 60,000 \\
\hline United States & 181,708 & 1,500 & 46,000 & 380 & 47,000 & 170,000 & 32,000 & 200,000 & 71 & 210,000 & 33,000 & 250,000 & 270,000 & 61,000 \\
\hline
\end{tabular}


[Million gallons per day]

\begin{tabular}{|c|c|c|c|c|c|c|c|c|c|c|c|c|c|c|}
\hline \multirow{4}{*}{ Region } & \multirow{4}{*}{$\begin{array}{c}\text { Popu- } \\
\text { lation } \\
1,000 \text { 's }\end{array}$} & \multirow{4}{*}{$\begin{array}{l}\text { Per } \\
\text { eapita } \\
\text { use } \\
\text { (gpd) }\end{array}$} & \multicolumn{11}{|c|}{ Water withdrawn } & \multirow{4}{*}{$\begin{array}{l}\text { Water } \\
\text { con- } \\
\text { sumed }\end{array}$} \\
\hline & & & \multicolumn{10}{|c|}{ Excluding irrigation conveyance losses } & \multirow{3}{*}{$\begin{array}{c}\text { Includ- } \\
\text { ing } \\
\text { convey- } \\
\text { ance } \\
\text { losses }\end{array}$} & \\
\hline & & & \multicolumn{3}{|c|}{ Ground water } & \multicolumn{3}{|c|}{ Surface water } & \multirow[b]{2}{*}{$\begin{array}{l}\text { Sew - } \\
\text { age }\end{array} \mid$} & \multicolumn{3}{|c|}{ All sources } & & \\
\hline & & & Fresh & Saline & $\begin{array}{l}\text { Fresh } \\
\text { and } \\
\text { saline }\end{array}$ & Fresh & Saline & $\begin{array}{l}\text { Fresh } \\
\text { and } \\
\text { saline }\end{array}$ & & Fresh & Saline & $\begin{array}{l}\text { Fresh } \\
\text { and } \\
\text { saline }\end{array}$ & & \\
\hline New England _ & 9,921 & 640 & 350 & 0 & 350 & 2,600 & 3,400 & 6,000 & 0 & 3,000 & 3,400 & 6,400 & 6,400 & 290 \\
\hline Delaware-Hudson. & 23,796 & 830 & 1,200 & 12 & 1,200 & 9,500 & 9,000 & 18,000 & 0 & 11,000 & 9,000 & 20,000 & 20,000 & 830 \\
\hline Chesapeake _. . . . & 8,663 & 820 & 480 & 0 & 480 & 4,700 & 1,900 & 6,600 & 70 & 5,200 & 1,900 & 7,100 & 7,100 & 320 \\
\hline South Atlantic .... & 14,539 & 910 & 2,000 & 0 & 2,000 & 7,500 & 3,800 & 11,000 & 0 & 9,500 & 3,800 & 13,000 & 13,000 & 1,500 \\
\hline Eastern Gulf $\ldots \ldots \ldots$ & 6,933 & 860 & 700 & 0 & 700 & 4,400 & 880 & 5,200 & 0 & 5,100 & 880 & 5,900 & 5,900 & 510 \\
\hline Tennessee-Cumberland & 4,184 & 1,800 & 370 & 0 & 370 & 7,200 & 0 & 7,200 & 0 & 7,500 & 0 & 7,500 & 7,500 & 400 \\
\hline Ohio & 17,950 & 1,300 & 1,300 & 34 & 1,300 & 22,000 & .3 & 22,000 & 0 & 24,000 & 34 & 24,000 & 24,000 & 800 \\
\hline $\begin{array}{l}\text { Eastern Great Lakes-St. } \\
\text { Lawrence. }\end{array}$ & 12,424 & 1,000 & 320 & 13 & 340 & 12,000 & 0 & 12,000 & 0 & 13,000 & 13 & 13,000 & 13,000 & 420 \\
\hline Western Great Lakes ... & 13,183 & 1,200 & 820 & 12 & 830 & 16,000 & 0 & 16,000 & 0 & 16,000 & 12 & 16,000 & 16,000 & 510 \\
\hline Hudson Bay & 666 & 260 & 44 & 6.0 & 50 & 120 & 0 & 120 & 0 & 160 & 6.0 & 170 & 170 & 54 \\
\hline Upper Mississippi _-_ & 12,359 & 920 & 1,300 & 20 & 1,300 & 10,000 & 0 & 10,000 & 0 & 11,000 & 20 & 11,000 & 11,000 & 570 \\
\hline Upper Missouri & 5,382 & 3,700 & 2,800 & 13 & 2,800 & 12,000 & 5.0 & 12,000 & 0 & 15,000 & 18 & 15,000 & 20,000 & 7,400 \\
\hline Lower Missouri .... & 2,451 & 640 & 180 & 0 & 180 & 1,400 & 0 & 1,400 & 0 & 1,600 & 0 & 1,600 & 1,600 & 200 \\
\hline Lower Mississippi_... & 4,735 & 1,100 & 1,300 & 25 & 1,400 & 23,000 & 1,600 & 3,900 & 0 & 3,700 & 1,600 & 5,300 & 5,300 & 1,300 \\
\hline Upper Arkansas-Red.- & 3,309 & 1,700 & 2,100 & 20 & 2,100 & 2,800 & 130 & 2,900 & 0 & 4,800 & 150 & 5,000 & 5,500 & 3,200 \\
\hline Lower Arkansas-Red-White & 3,884 & 1,300 & 920 & 38 & 960 & 3,900 & 4.0 & 3,900 & 0 & 4,800 & 42 & 4,900 & $4,9.00$ & 850 \\
\hline Western Gulf & 10,029 & 2,200 & 11,000 & 14 & 11,000 & 6,600 & 1,400 & 8,000 & 0 & 17,000 & 1,500 & 19,000 & 22,000 & 9,200 \\
\hline Colorado ............ & 2,027 & 7,000 & 3,500 & 6.3 & 3,500 & 8,200 & 0 & 8,200 & .2 & 12,000 & 6.3 & 12,000 & 14,000 & 7,100 \\
\hline Great Basin $\ldots \ldots$ & 1,204 & 580 & 910 & 6.0 & 910 & 4,900 & 5.5 & 4,900 & 0 & 5,800 & 12 & 5,800 & 7,000 & 3,300 \\
\hline South Pacific & 15,412 & 2,100 & 10,000 & 140 & 10,000 & 9,400 & 9,100 & 19,000 & .5 & 20,000 & 9,300 & 29,000 & 33,000 & 13,000 \\
\hline Pacific Nor thwest & 5,412 & 5,400 & 3,700 & 0 & 3,700 & 18,000 & 48 & 18,000 & 0 & 22,000 & 48 & 22,000 & 29,000 & 8,300 \\
\hline Hawaii & 634 & 2,500 & 580 & 21 & 600 & 600 & 260 & 860 & 0 & 1,200 & 280 & 1,500 & 1,600 & 410 \\
\hline Alaska & 226 & 870 & 25 & 0 & 25 & 170 & 0 & 170 & 0 & 200 & 0 & 200 & 200 & .7 \\
\hline Puer to Rico and Virgin Islands & 2,385 & 510 & 210 & 1.6 & 210 & 320 & 650 & 970 & 0 & 530 & 650 & 1,200 & 1,200 & 280 \\
\hline $\begin{array}{l}\text { United States excluding Alaska, } \\
\text { Hawaii, Puerto Rico and } \\
\text { Virgin Islands. }\end{array}$ & 178,463 & 1,500 & 46,000 & 360 & 46,000 & 170,000 & 31,000 & 200,000 & 71 & 210,000 & 32,000 & 240,000 & $270,000 \mid$ & 60,000 \\
\hline United States & 181,708 & 1,500 & 46,000 & 380 & 47,000 & 170,000 & 32,000 & $200 ; 000$ & 71 & 210,000 & 33,000 & 250,000 & 270,000 & 61,000 \\
\hline
\end{tabular}




\section{NONWITHDRAWAL USES}

Nonwithdrawal uses do not lend themselves to evaluation of the quantity of water used. These uses, however, do have a very large economic value and may have an appreciable effect on the quantity and quality of water available for other uses. The most important nonwithdrawal uses are navigation, waste disposal, recreation, and conservation of fish and wildlife.

The consumption of water by nonwithdrawal uses in the United States has not been estimated; however, fresh water evaporation in the 17 Western States has been estimated. According to Meyers (1962), annual evaporation in the 17 Western States amounts to 24,000 acre-feet per year or an average rate of 21,000 mgd. This is an amount equal to the water used by all public supplies in the United States in 1960 and equal to 18 percent of the fresh water withdrawn and 41 percent of the water consumed in these States. Evaporation from all water surfaces should be an approximate measure of consumption by nonwithdrawal use.

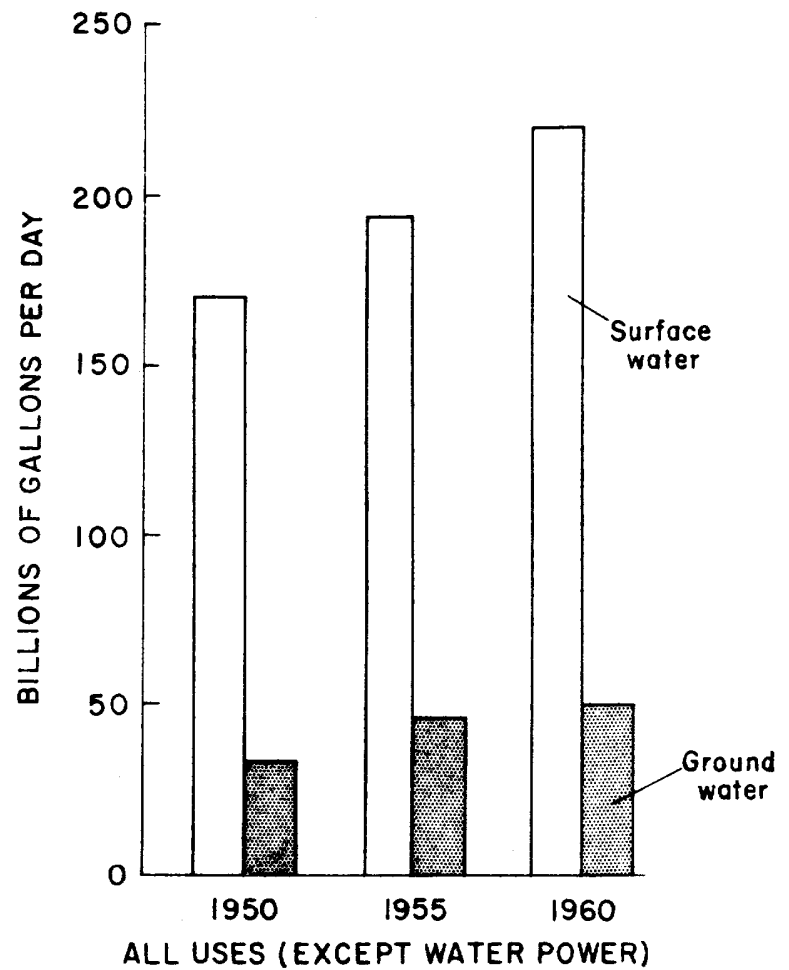

\section{CHANGES SINCE 1955}

The present survey is comparable to the 1950 and 1955 surveys (MacKichan, 1951 and 1957) and the 1945 survey of ground water (Guyton, 1950). The 1950 survey did not include irrigation conveyance losses nor did it include water-use data tabulated by regions. The withdrawal of water for all uses except waterpower increased 12 percent since 1955 (see table 17). The use of water for waterpower increased 33 percent. The use of selfsupplied industrial water increased 27 percent and the use of water for public supplies increased 23 percent. Rural use was about the same in 1960 as in 1955.

During the last 5 years the use of surface water, exclusive of that for waterpower, increased from 190,000 to $220,000 \mathrm{mgd}$, and the use of ground water increased from 46,000 to $47,000 \mathrm{mgd}$. The percentage increase for surface water was 16 percent as compared with 2 percent for ground water. Earlier surveys did not include estimates of water consumed, but trends in consumptive use would probably be similar to trends in

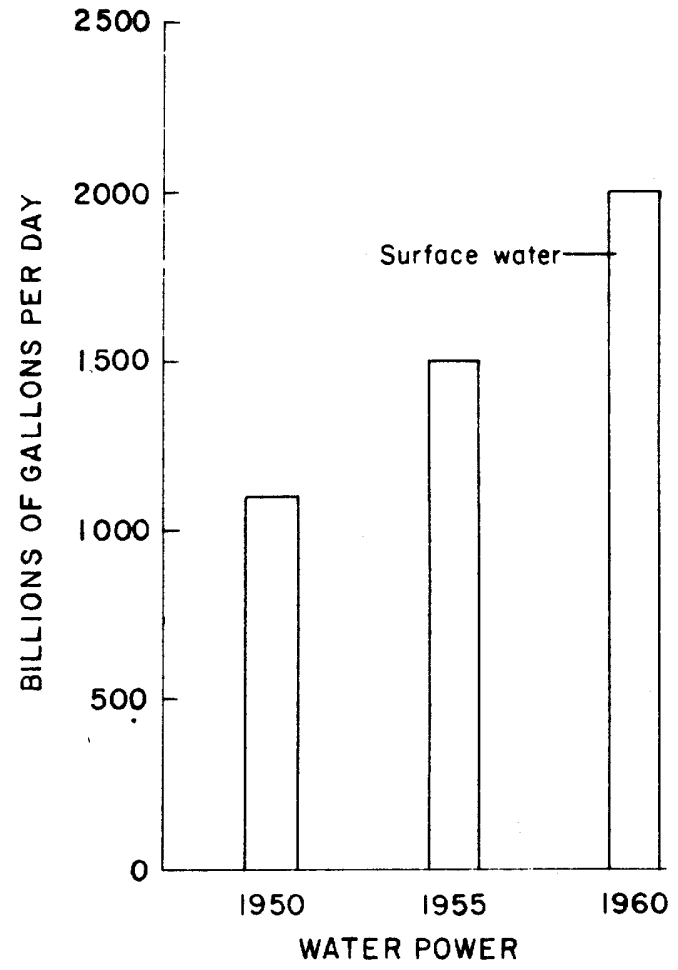

Figure 7. - Trends in use of water for waterpower and all other uses. 

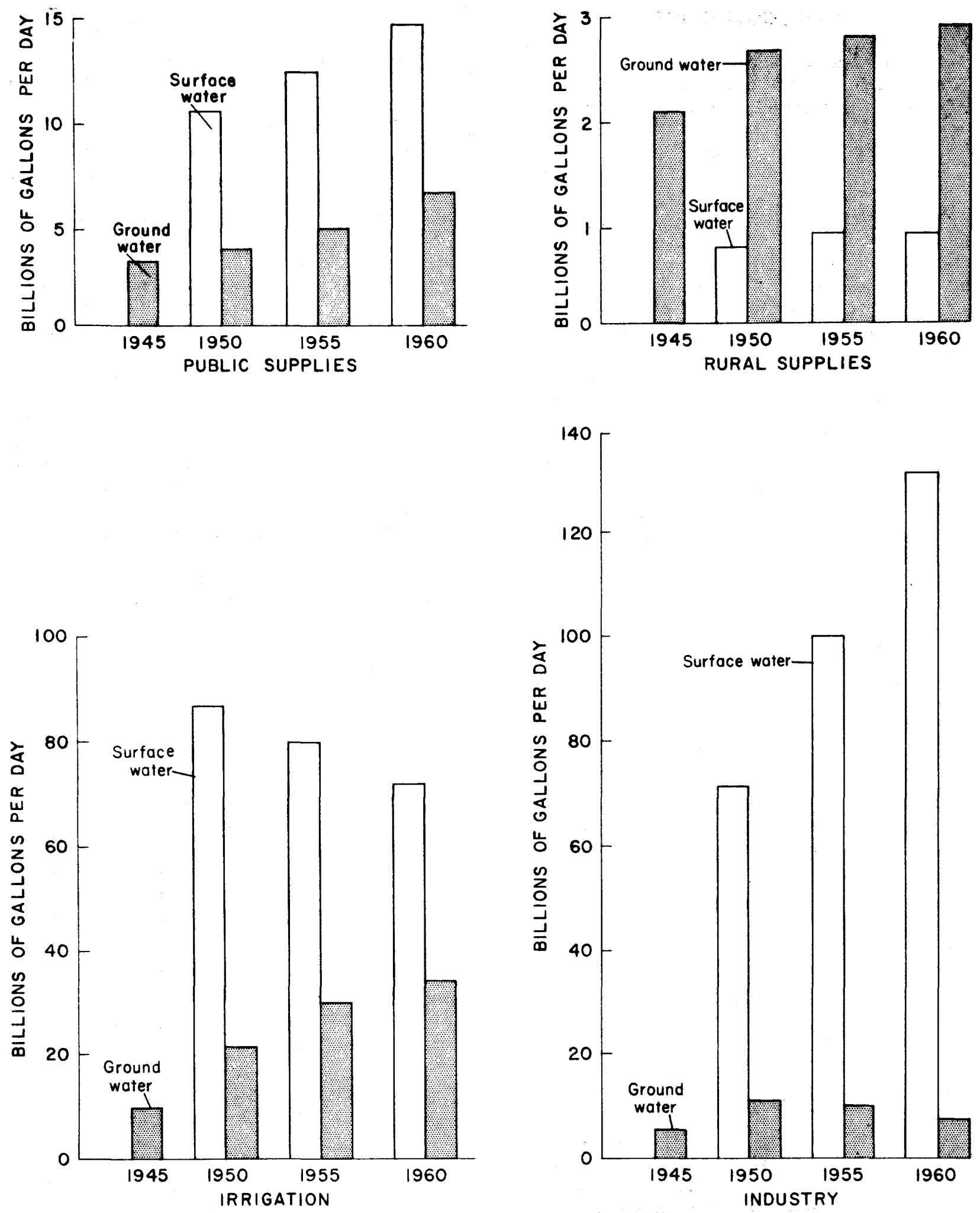

Figure 8. - Trends in water used for public supplies, rural supplies, irrigation, and industry. Note: Surface-water data not available in 1945. 
Table 17.-Change in withdrawals, 1950-1960

\begin{tabular}{|c|c|c|c|c|}
\hline & $\begin{array}{c}1950 \\
(\mathrm{mgd})\end{array}$ & $\begin{array}{c}1955 \\
\text { (mgd) }\end{array}$ & $\begin{array}{c}1960 \\
\text { (mgd) }\end{array}$ & $\begin{array}{c}\text { Percent } \\
\text { increase } \\
1955 \text { to } \\
1960\end{array}$ \\
\hline Rural & 3,600 & $1_{3,600}$ & 3,600 & 0 \\
\hline Public supplies. & 14,000 & 17,000 & 21,000 & 23 \\
\hline Self-supplied industrial & 77,000 & 110,000 & 140,000 & 27 \\
\hline Irrigation (except conveyance losses) & 79,000 & 81,000 & 84,000 & 4 \\
\hline Irrigation (including conveyance losses) & $-\ldots$ & 110,000 & 110,000 & 0 \\
\hline All uses (excluding conveyance losses) & 170,000 & 210,000 & 250,000 & $-\cdots-\ldots$ \\
\hline All uses (including conveyance losses) & 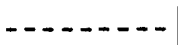 & 240,000 & 270,000 & 12 \\
\hline Waterpower & $1,100,000$ & $1,500,000$ & $2,000,000$ & 33 \\
\hline
\end{tabular}

${ }^{1}$ Revised.

withdrawals. The trend in total water withdrawn has been upward. (See fig. 7.) However, there is some evidence that the rate of increase is declining in some areas and in some categories. (See fig. 8.) Although the accuracy of some parts of the 1950,1955 , and present estimates may be rather poor, the downward trends in surface water used for irrigation and in ground water used by industry are too well defined to be ignored. Ground water used for irrigation shows an equally well-defined upward trend. The great increase in surface water used by industry is mostly in cooling water for fuel-electric powerplants.

Part of the downward trend is caused by economies in the use of water. For example, many petroleum refineries in the Delaware River basin have installed cooling towers during the last few years, which reduce their water intake but increase the water consumed slightly. Rates of water use in some localities have declined because of reduced production, such as by the steel industry, or because industries using large quantities of water have moved to other areas.

\section{SUPPLY VERSUS DEMAND}

Much of the withdrawn water is not consumed but is returned to a downstream or underground source after use; therefore, the total supply is not depleted by the entire amount withdrawn. For this reason the quantity of water withdrawn is not directly comparable with the supply and, in fact, may exceed the total supply, owing to repeated use.
On the other hand, water that is consumed in its use is no longer available; consequently, consumptive use can be compared with supply.

The dependable supply is not a fixed amount but can be increased by surface storage, artificial recharge of ground water, and by reducing evapotranspiration losses or other forms of waste. These measures increase the cost of water; therefore, the increase in dependable supply depends on the ability and willingness of the user to pay the higher cost.

. The long-term average runoff of a river basin, with few exceptions, is the upper limit of possible production of the combined surface- and ground-water resources of the basin. The exceptions are in the narrow. fringes along the coasts where some water that percolates underground directly into the ocean could be used without affecting runoff, provided such use does not cause contamination by sea water. Runoff is the total flow of a stream including outflow from groundwater storage through springs and seeps as well as the overland flow that follows hard rains.

The runoff of all streams in the United States, exclusive of Alaska and Hawaii, averages $1,200,000 \mathrm{mgd}$, but it is not uniformly distributed throughout the Nation (table 18). The average annual runoff ranges from less than one-fourth inch in.several places in the Southwest to more than 80 inches at some places along the Pacific Coast. In addition to this great areal variation, runoff varies widely from day to day and year to year in 
Table 18. - Supply compared with demand, 1960

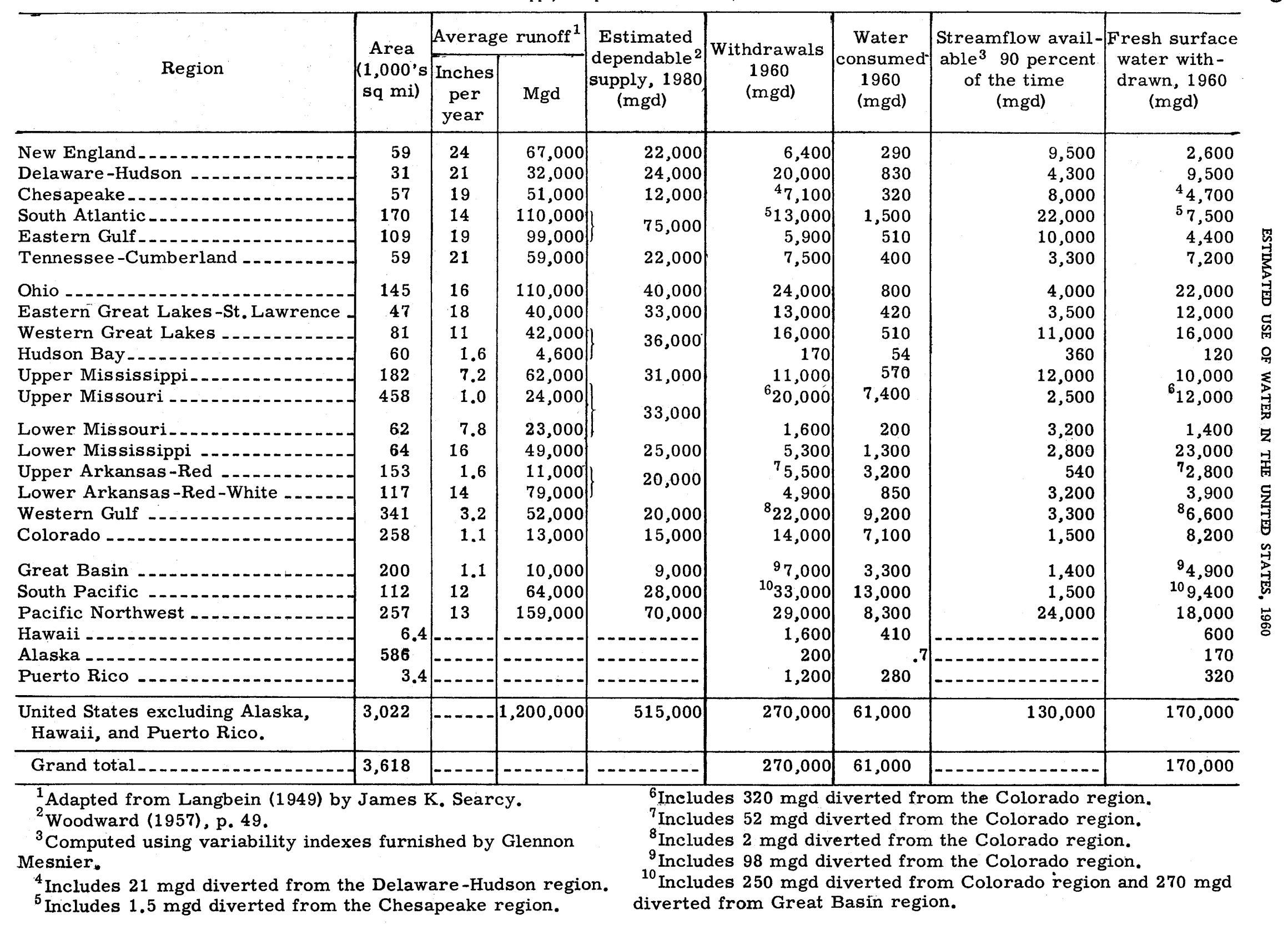


any one locality. Some of the runoff which occurs during times of flood cannot be made available for water supply for economic and sometimes technical reasons.

There is no simple method of computing the combined dependable supply of surface water and ground water. The flow, available 90 percent of the time without storage (table 18), is used by some as an index to the practicable dependable streamflow; but even this quantity of water is not available 100 days out of every thousand. This quantity of water can be made available all the time by supplementing the natural streamflow with releases from reservoirs. Even greater flows may be made dependable by increased storage; however, the benefits derived from storing water follows a law of diminishing returns (Langbein, 1959). Each increment of controlled flow requires a larger amount of reservoir storage space than the preceeding increment, and consequently each increment costs more than the preceding one. Further more, with each increment of controlled flow, the surface area of the reservoir is increased, which permits additional evaporation. Storage capacity in abasin may become so great that the most recent increment of

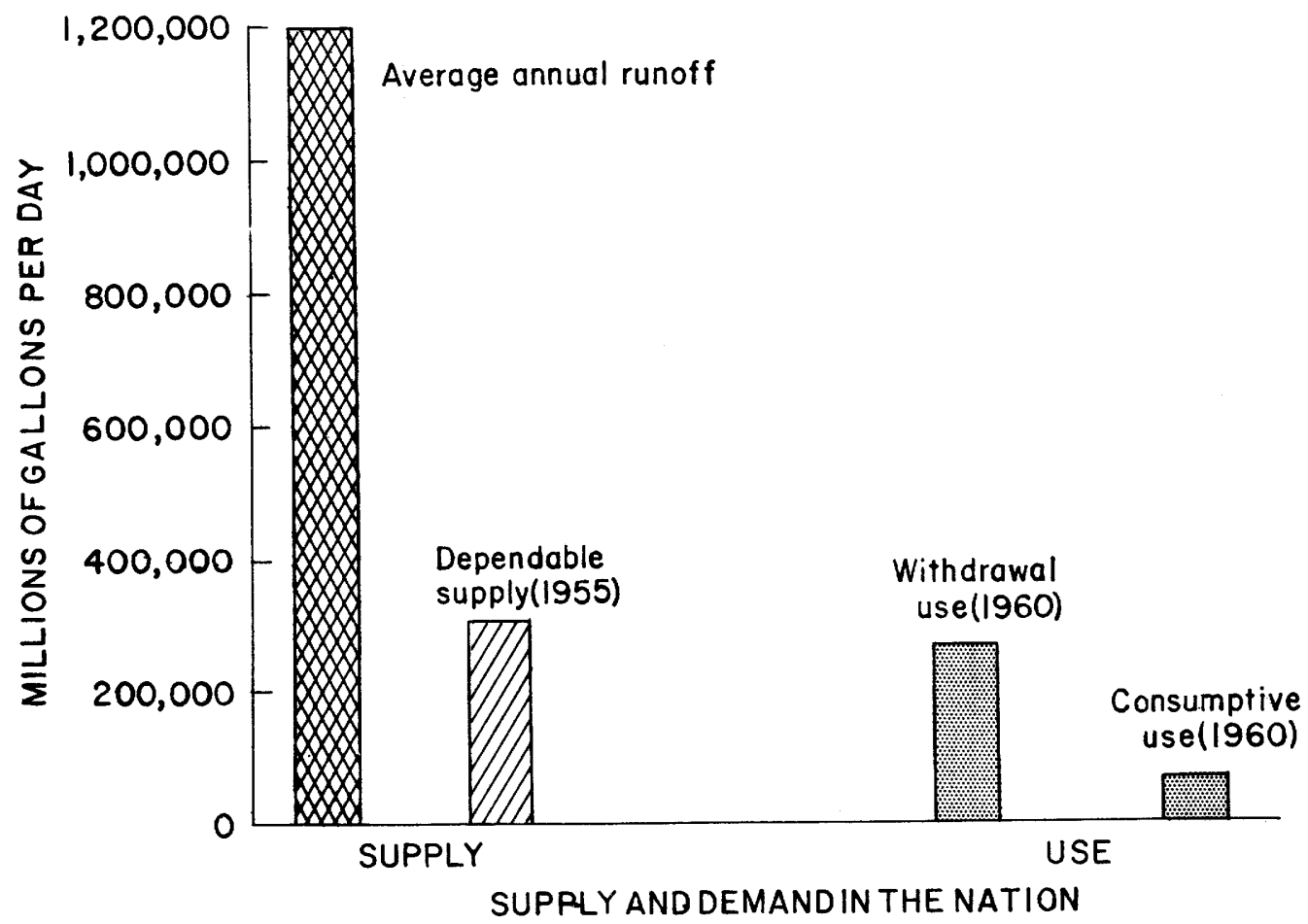

stored water will be dissipated by evaporation.

Woodward (1957) estimated that the economically dependable supply was $314,000 \mathrm{mgd}$ in 1955 . He also estimated that the probable dependable supply would be $515,000 \mathrm{mgd}$ by 1980. The increase in dependable supply from 314,000 to $515,000 \mathrm{mgd}$ would be accomplished by providing additional surface storage, by using artificial recharge, and by salvaging waste water by reducing evapotranspiration.

Figure 9 shows that the Nation is far from running out of water. Consumptive use is only 20 percent of Woodward's estimated de pendable supply in 1955. However, withdrawal uses in 1960 were approaching Woodward's estimate of supply.

Although the outlook in the West is less promising (fig. 10) there is still room for further development. Present withdrawals are about equal to Woodward's estimate of the 1955 dependable supply but considerably less than the ultimate supply (average runoff). Woodward estimates that the dependable supply by 1980 will be almost $200,000 \mathrm{mgd}$ or 


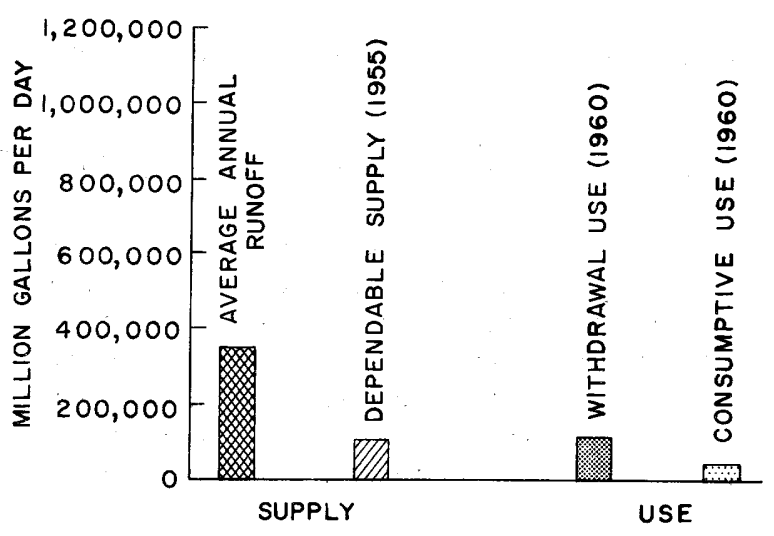

Figure 10. - Supply and demand in the West.

about $70,000 \mathrm{mgd}$ greater than in 1955. Present withdrawals are 120,000 mgd, of which $52,000 \mathrm{mgd}$ is consumed.

The fresh surface water used in the Nation as a whole may be properly compared with the streamflow which is available 90 percent of the time. During 1960,170,000 mgd of fresh surface water was used, whereas the streamflow available amounted to only $130,000 \mathrm{mgd}$. Some of the $170,000 \mathrm{mgd}$ was withdrawn from storage and some of the water was used two or more times. The above comparison does not include water for developing waterpower. Table 18 shows that the streamflow available 90 percent of the time was less than the quantity of surface water used in several regions.

\section{SELECTED REFERENCES}

American Water Works Association, 1948, A survey of operating data for water works in 1945: Am. Water Works Assoc. Jour., v. 40 , no. 2 , p. $167-260$.

1953, A survey of operating data for water works in 1950: Am. Water Works Assoc. Jour., v. 45, no. 6, p. 583-678.

-1957, A survey of operating data for water works in 1955: Am. Water Works Assoc. Jour., v. 49, no. 5, p. 555-696.

American Water Works Association Commit tee on Water Use in Air Conditioning and Other Refrigeration, 1958, Trends in airconditioning use regulation: Am. Water Works Assoc. Jour., v. 50, no. 1, p. 7596.

American Water Works Association Task Group, 1953, Water conservation in indus try: Am. Water Works Assoc. Jour., v. 45, no. 12 , p. $1249-1260$.
Arkansas-White-Red Basins Inter-Agency Committee, 1957, Development of water and land resources of the Arkansas-WhiteRed River basins: 85th Cong., 1st sess., S. Doc. 13 .

California Water Resources Board, 1955, Water utilization and requirements of California: California Water Resources Board Bull. 2, v. I, 227 p.; v. II, 358 p.

Connecticut Water Resources Commission, 1957, Water resources of Connecticut, a report to the General Assembly: 200 p.

Foley, F. C., Smrha, R. V., and Metzler, Dwight, 1955, Water in Kansas: Report to the 1955 State Legislature, 216 p.

Frank, Bernard, 1955, The story of water as the story of man: U.S. Dept. Agriculture Yearbook-Water, 1955, p. 1-8.

Guyton, W.F., 1950, Estimated use of ground water in the United States, 1945: U.S. Geol. Survey open-file report.

Indiana Water Resources Study Committee, 1956, Indiana water resources: 44 p., 13 figs., 2 tables.

Kentucky Water Resources Commission, 1959, Kentucky water resources study: Dept. Conserv., Div. Flood Control and Water Usage, $109 \mathrm{p}$.

Krieger, R.A., Hatchett, J.L., and Poole, J.L., 1957, Preliminary survey of the salinewater resources of the United States: U.S. Geol. Survey Water-Supply Paper 1374, $172 \mathrm{p}$.

Langbein, W. B., 1950, An American survey: Water Power, v. 2, no. 6, London, p. 238-245. 1959 , Water yield and reservoir storage in the United States: U.S. Geol. Survey Circ. 409,5 p.

Langbein, W.B., and others, 1949, Annual runoff in the United States: U.S. Geol. Survey Circ. $52,14 \mathrm{p}$.

Mangan, J.W., and Graham, J.B., 1953, The use of water in Pennsylvania, 1951: U.S. Geol. Survey Circ. 257, 11 p.

MacKichan, K. A., 1951, Estimated use of water in the United States, 1950 : U.S. Geol. Survey Circ. 115, 13 p.

1957, Estimated use of water in the United States, 1955: U.S. Geol. Survey Circ。 $398,18 \mathrm{p}$.

MacKichan, K. A., and Kammerer, J. C., 1961, Preliminary estimates of water used, Southeast river basins, 1960: U.S. Geol. Survey Circ. 449.

Marion, A. W., 1952, The water situation and Ohio agriculture, in the Proceedings of the First Ohio Water Clinic, Ohio State Univ.: Eng. Expt. Sta. Bull. 147, p. 21-26. 
Meyers, J.S., 1962, Evaporation from the 17 Western States, with a section on Evaporation rates, by Nordenson, T. J.: U.S. Geol.Survey Prof. Paper 272-D, 76 p., (in préss). Piction, W. L., 1952, The national picture: Ill. State Water Survey Bull. 41, p. 127-130. -1956, Water use in the United States, 1900-1975: U.S. Dept. Commerce, Business and Defense Services Adm., Business Service Bull. 136, 6 p.

1960, Water use in the United States, 1900-1980: U.S. Dept. Commerce, Business and Defense Services Adm., 6 p.

Rudnick; A. R., 1959, Water use in Ohio: Ohio Dept. of Natural Resources, Div. Water, Ohio Water Plan Inventory Rept. 6, 50 p., 6 pls., 10 tables.

Seidel, H.F., and Carpenter, J. M., 1958, Relationships of temperature and air-conditioning water use: Am. Water Works Assoc. Jour., v. 50, no. 2, p. 226-232.

Smith, J. G., Haber; R. A., Kaplovsky, A. J., and Simpson, C. O., 1960, Intrastate water resources survey, 1959: Delaware members of the Delaware Basin Survey Coordinating Comm., $432 \mathrm{p}$.

Sykes, J.F., 1955, Animals and fowl and water: U.S.Dept. Agriculture Yearbook-Water, 1955, p. 14-18.

Temporary (New York) State Commission on Water Resources Planning, 1960, Dynamic planning-First step in water resources development for New York State: Legislative Doc. 24, $260 \mathrm{p}$.

Tennessee Water Resources Division, 1961, Tennessee's water resources: $128 \mathrm{p}$.

Thomson, M.T., Herrick, S.M.,Brown, Eugene, and others, 1956, Availability and use of water in Georgia: Georgia Dept. of Mines, Mining, and Geology Bull. 65, 316 p.

U.S. Dept. of Agriculture, 1960, Livestock and poultry inventory, January 1: Agr. Marketing Service, Crop Reporting Board, Ann. Rept. $30 \mathrm{p}$.

U.S. Bureau of the Census, 1952, U.S. Census of agriculture: 1950; V. III, Irrigation of Agricultural Lands.

-1955, Water use in Manufacturing: 1953: U.S. Bur. Census Rept. MAS-53-3.

-1956, U.S. census of agriculture: 1954; v. I, Counties and State economic areas: -1957, U.S. census of manufactures: 1954: v. 3 .

1960 a, U.S. census of manufactures: 1954; Industrial water use supplement: U.S. Bur. Census Bull. MC-209 (supp.), 51 p. 1958 .
U.S. Bureau of the Census, 1960c, 1959, census of agriculture.

U.S. Federal Power Commission, 1957a, Hydroelectric power resources of the United States, developed and undeveloped, 1957: FPC P-32, 196 p.

- $1957 \mathrm{~b}$, Water requirements of utility steam electric generating plants-1954: Rept. FPC-DC-57.

1960-61, Production of electric energy and capacity of plants, section 1, in Electric power statistics: FPC-DC, Rept. nos. 60-1A to $60-12 \mathrm{~A}$.

U.S. Public Health Service, 1948, Inventory of water and sewage facilities in the United States, 1945.

1954 , Inventory of municipal and indus trial waste facilities in the United States, 1953.

-1955a, Inventory of municipal water facilities for larger communities, 1954 revised.

1955b, Municipal water facilities, communities of 25,000 population and over, continental United States, as of December 31, 1954, revised: $153 \mathrm{p}$.

1956, Municipal water facilities, communities of 25,000 population and over, continental United States, as of December 31, 1955: $153 \mathrm{p}$.

1957, Municipal water facilities, communities of 25,000 population and over, continental United States and territorial possessions, as of December 31, 1956: $163 \mathrm{p}$.

-1959a, Federal programs for collection of data on water use: Subcomm. on Hydrollogy, Inter-Agency Comm. on Water Resources, Notes on Hydrol. Activities Bull. $10,43 \mathrm{p}$.

- $1959 \mathrm{~b}$, Municipal water facilities, communities of 25,000 population and over, continental United States and territorial possessions, as of January 1, 1958: Public Health Service Pub. 661, 83 p.

-1960, Municipal water facilities, communities of 25,000 population and over, United States and possessions, as of January 1, 1960: Public Health Service Pub. 661 (1960 ed.), 91 p.

-1960-61, Municipal water facilities inventory as of January 1, 1958: U.S. Public Health Service Pub. 775, v. 1-9.

U.S. Senate, Select Committee on National Water Resources, 1959a, Water resources activities in the United States: U.S. 86th Cong., 1st sess., Comm. print 1, 59 p. 
U.S. Senate, Select Committee on National Water Resources, 1959b, National water resources and problems: U.S. 86th Cong., $2 \mathrm{~d}$ sess., Comm. print 3,42 p., 43 figs., 13 tables.

1960a, Future water requirements for municipal use: U.S. 86th Cong., 2d sess., Comm. print 7, $24 \mathrm{p}$.

$-1960 \mathrm{~b}$, Future water requirements of principal water-using industries: U.S. 86th Cong., 2d sess., Comm. print 8, 101 p.

$-1960 c$, Electric power in relation to the Nation's water resources: U.S. 86th Cong., 2d sess., Comm. print 10, 86 p.

$-1960 d$, Estimated water requirements for agricultural purposes and their effects on water supplies: U.S. 86th Cong., 2d sess., Comm. print 13, 34 p.
U.S. Senate, Select Committee on National Water Resources, $1960 \mathrm{e}$, Water supply and demand: U.S. 86th Cong., 2 d sess., Comm. print $32,131 \mathrm{p}$.

Virginia Advisory Legislative Council, 1955, Water resources of Virginia: Richmond, Va., Rept. to Governor and General Assembly, 139 p.

Wirth, H. E., 1959, Water use in Wisconsin: Wisconsin State Board Health, 36 p., [bibliography].

Wood, Noel H., 1959, Arkansas water resources: Arkansas Univ. Coll. Business Adm., Indus. Research Ext. Center, 180 p. Woodward, D. R., 1957, Availability of water in the United States with special reference to industrial needs by 1980 : Industrial College of Armed Forces, Washington, D.C., 74 p. 